\title{
Einfluss des Wirtswechsels der Kohlmotte, Plutella xylostella L. auf Erbsen auf ihre natürlichen Feinde in Kenia
}

Influence of the Host Shift of the Diamondback Moth, Plutella xylostella L. to Peas on its Parasitoids in Kenya

\author{
Dissertation \\ zur Erlangung des Doktorgrades \\ der Fakultät für Agrarwissenschaften \\ der Georg-August-Universität Göttingen \\ vorgelegt von \\ Andrea Rossbach \\ geboren in Marl
}

Göttingen, den 15. November 2005 
Referent: Prof. Dr. S. Vidal

Koreferent: Prof. Dr. M. Pöhling 


\section{Contents}

Abstract

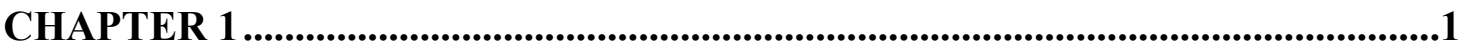

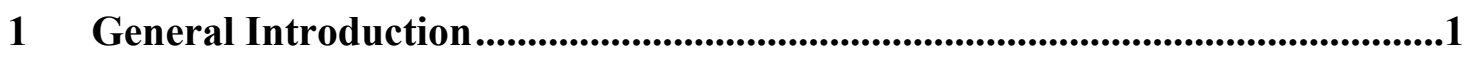

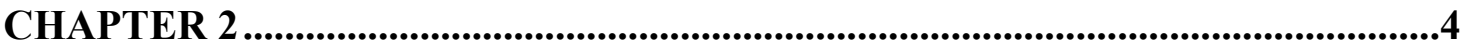

2 Host shift to peas in the diamondback moth Plutella xylostella $\mathrm{L}$. and response of its parasitoid Diadegma mollipla ............................................................................4

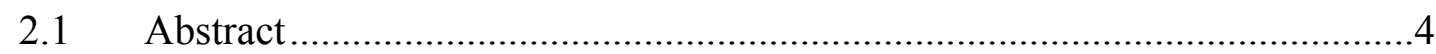

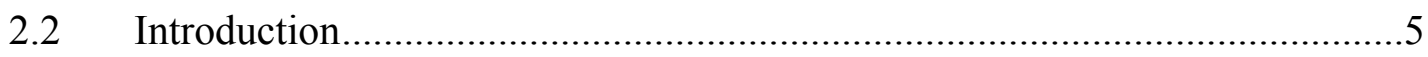

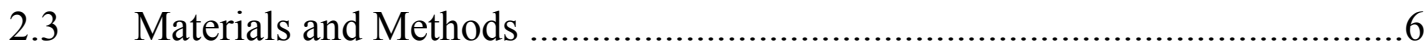

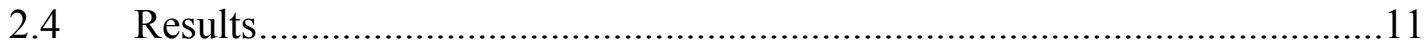

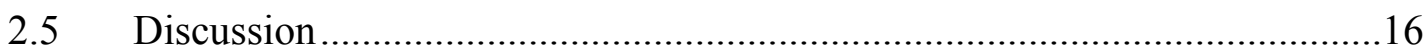

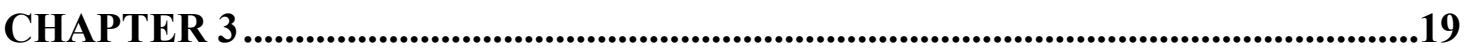

3 Generalism versus specialism: Responses of Diadegma mollipla (Holmgren) and Diadegma semiclausum (Hellen), to the host shift of the diamondback moth

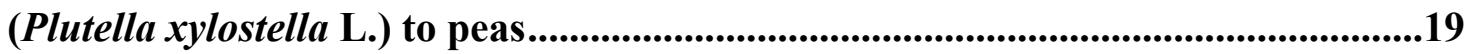

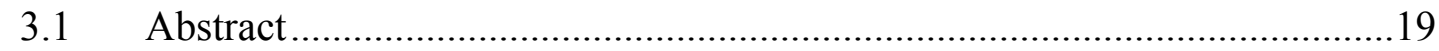

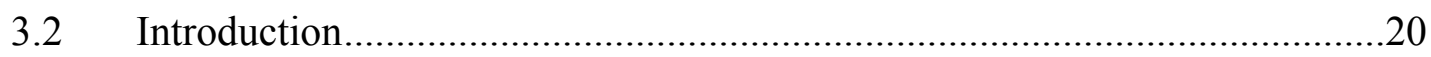

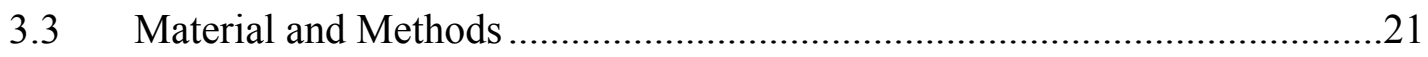

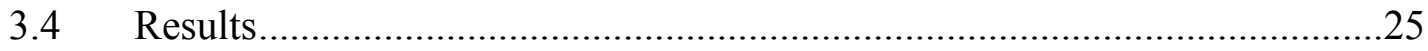

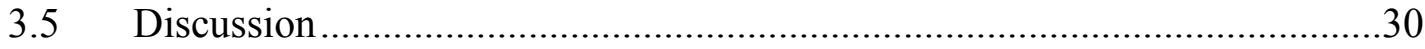

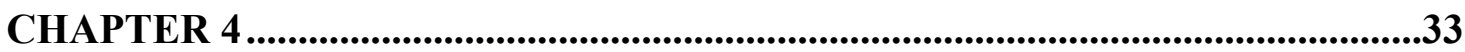

4 Does a specialist parasitoid adapt to its host on a new host plant?..................33

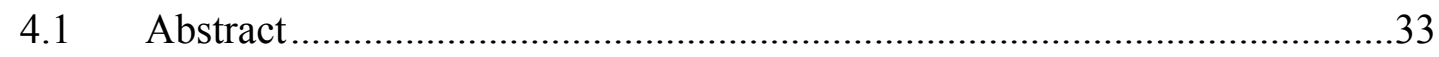

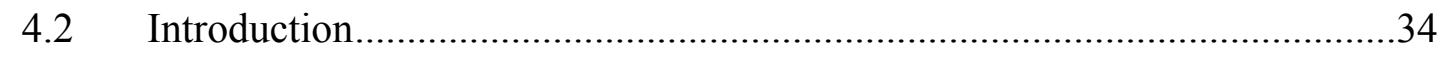

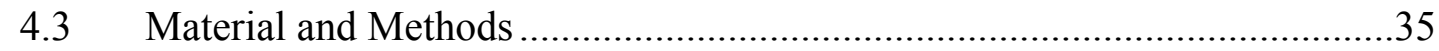

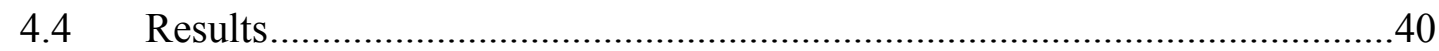

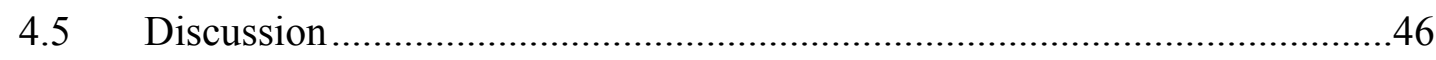

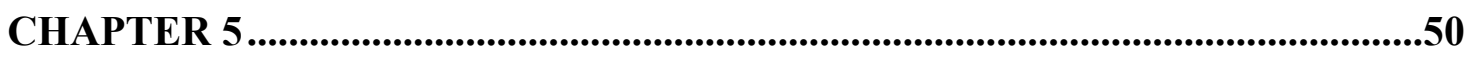

5 Interspecific competition between Diadegma semiclausum Hellen and Diadegma mollipla (Holmgren) on Plutella xylostella (L.) feeding on an unusual host plant. 


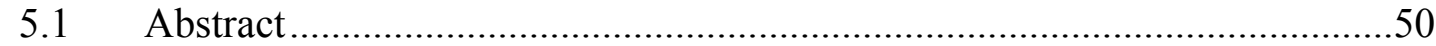

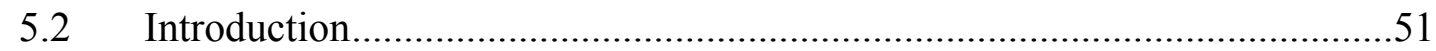

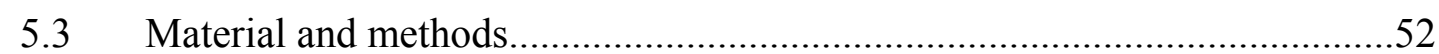

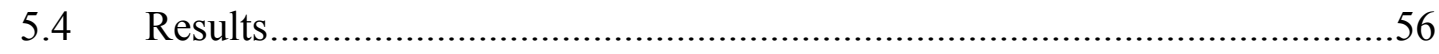

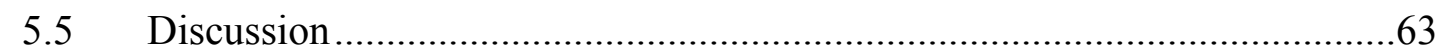

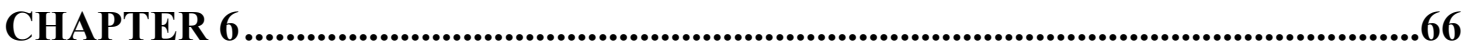

6 Parasitoid diversity of Plutella xylostella L. feeding on an unusual host plant66

\begin{tabular}{|c|c|}
\hline 6.1 & (1) \\
\hline 6.2 & 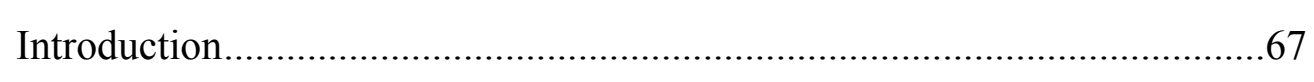 \\
\hline 6.3 & Material and Methods \\
\hline 6.4 & Results \\
\hline 6.5 & 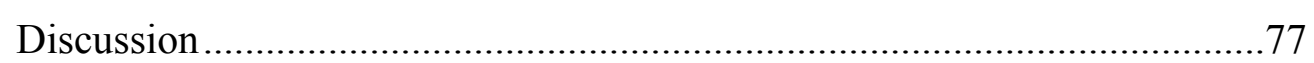 \\
\hline
\end{tabular}

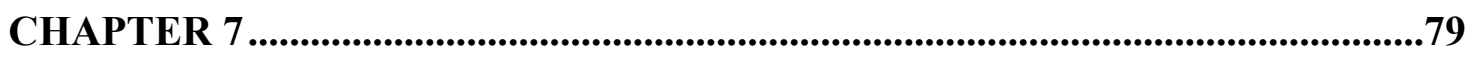

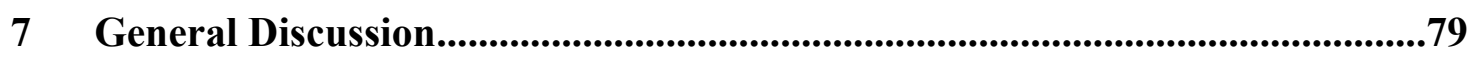

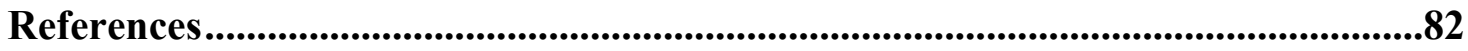

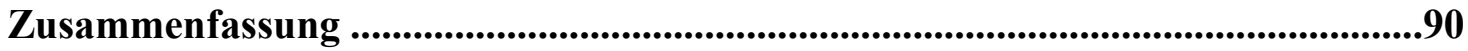

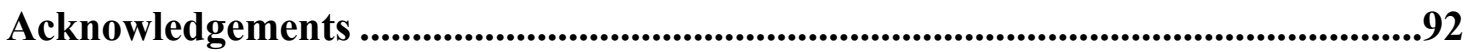

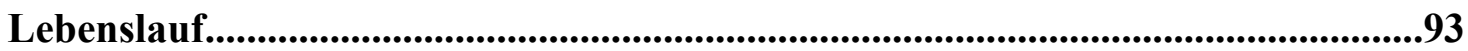




\section{Abstract}

A population of the diamondback moth (DBM), Plutella xylostella L., known as an oligophagous pest on crucifers was recently found to infest sugar snap- and snowpeas (Pisum sativum L.) in the Rift Valley in Kenya, causing heavy damage. The impact of this host shift on the interaction with associated parasitoids was investigated. In laboratory experiments parasitation levels, development and survival, and the role of host plants for host location of two important parasitoids were studied: The indigenous Diadegma mollipla (Holmgren), regarded as a relative generalist, and the highly specific Diadegma semiclausum (Hellen), introduced to Kenya for biocontrol of DBM on cruciferous vegetables.

Tested individually $D$. mollipla surprisingly proved to be more effective on the new host plant than on cabbage. Diadegma semiclausum parasitized more efficiently on DBM on cabbage. Bioassay tests, conducted with a Y-tube olfactometer, showed that the specialisation of $D$. semiclausum is mediated by host plant signals, associated with crucifers, which are not encountered in DBM feeding on peas. Whereas for D. mollipla crucifer volatiles seem not to be used as primary cues for host location. Differential performance and host location abilities of the two parasitoids suggested a difference in competitiveness on peas as compared to cabbage. On cabbage, D. semiclausum clearly out-competes its congenus. On peas, confined to small containers D. mollipla parasitized equally as good or even better than D. semiclausum. However, under more natural conditions in the greenhouse, D. semiclausum parasitized significantly more larvae than its competitor on both host plants. Chemical interference between the two species could be a possible explanation.

Olfactory learning is known to enable parasitoids to modify their behavioural responsiveness to host and plant- derived odours. In view of increasing the potential of D. semiclausum for biocontrol of DBM in peas, the parasitoid was reared for three subsequent generations on the pea strain of DBM. Adaption to the new host plant through pre-emergence learning seemed to be possible. Response to DBM-infested pea and levels of parasitation increased in the pea generations. However, fitness trade-offs, especially an extreme shift in sex ratio to males reduced reproductive success of $D$. semiclausum on peas. 
In order to investigate the effect of the unusual host plant on the entire local parasitoid community associated with DBM, field studies were conducted close to the farm where DBM was first discovered on peas. Peas provided an enemy-free space for DBM. Local parasitoids occurred only sporadically and in very low numbers on DBM on peas. The release of $D$. semiclausum in the study site led to a reduction of DBM in kales. It did not establish in peas. The number of local parasitoids gradually decreased after the introduction. With a strong competition on kales especially D. mollipla was assumed to find a niche in peas. However, it did not occur in larger numbers in peas. The species might have moved to other host instead.

We demonstrated, that the host plant had a strong influence on parasitation, fitness and host loaction. The inclusion of a new host plant into its feeding repertoire enables the herbivore host to avoid enemy attack. But due to the ability to adapt to a new host plant, parasitoids could follow their host with time. 


\section{CHAPTER 1}

\section{General Introduction}

The diamondback moth, Plutella xylostella L. (Lepidoptera: Plutellidae) is not only the most damaging pest of crucifers worldwide but also the most difficult to control (Talekar and Shelton, 1993). It is considered as an oligophagous pest feeding only on the plant family of Brassicaceae. It was believed to have originated in the Mediterranean region (Harcourt, 1956), the origin of cultivated brassicas. Recently this has been questioned and southern Africa was suggested as the origin of the diamondback moth, due to the richness and high diversity of parasitoids and a large number of indigenous plants from the Brassicaceae found (Kfir, 1998).

In Kenya a number of crucifereous vegetables are grown. Main vegetables are kales and cabbages which are produced for home consumption and domestic markets. In the past, farmers depended solely on insecticide use to control DBM. Increasing resistence against most pesticides led to increased spraying intervals. Production became uneconomic on the one hand and high pesticide residues threatened the health of farmers and consumers as well as the environment. Therefore ICIPE (The International Centre of Insect Physiology and Ecology based in Nairobi) started a project on "Development of a biocontrol-based IPM for the diamondback moth in eastern and southern Africa". Coinciding with the beginning of the project, vegetable growers in Naivasha in the Rift Valley of Kenya complained about DBM attacking sugar snap-and snowpeas (Pisum sativum L.). At first this news was met with disbelief, because this plant was completely outside the normal host range, but larvae, pupae and moths were unmistakably those of Plutella xylostella L. and the identification was confirmed by a specialist, Koen Maes of the Tevuren Museum in Belgium (Löhr, 2001). The DBM population that was detected on peas on the commercial farm in Naivasha turned out to be a new DBM strain with biological differences. While larvae of the cabbage strain cannot normally survive on peas, the pea strain developed equally well on both host plants. Development was slightly slower though and pupae were smaller as compared to the cabbage strain (Löhr and Gathu, 2002). 
Despite the common believe that DBM is restricted to cruciferous plants, the species has occasionally been found on other host plants. It has been reported on chickpea (Cicer arietinum L.) and a chenopodeaceous vegetable Salsola kali in Russia (Reichert, 1919, in Talekar et al., 1985) and on okra in Ghana (Anonymous, 1971). On peas occasional occurences of DBM have been reported from Taiwan and the Philippines (Talekar, pers. comm.), but no published information exists.

This phenomenon was the rare opportunity to investigate the influence of a host plant shift on herbivore-associated parasitoids. Historically, plant chemistry and physiology were seen as the determinant for host plant specifity in phytophagous insects (Ehrlich and Raven, 1964). The hypothesis of enemy-free space by Jeffries and Lawton (1984) drew the attention to the importance of natural enemies on host plant selection by herbivores. One way to escape natural enemies would be the utilization of novel host plants that provide an ecological refuge from enemies. Recently several studies have demonstrated the existence of enemy-free space through the acquisition of a new host plant (Brown et al., 1995, Feder, 1995, Gratton and Welter, 1999, Gross et al., 2004, Mulatu et al., 2004). Herbivores were able to overcome physiological trade-offs on the novel plant in order to avoid parasitoid attack.

So how does a host shift affect parasitoids? Chemical and morphological plant attributes can influence the foraging success of parasitoids as well as the role of plants as host finding cues (Cortesero et al., 2000). The shift to a novel host plant can result in a loss of plant-related cues and to a reduced searching efficiency of especially specialist parasitoids (Brown et al., 1995). When feeding on different food plants physiology and size of the herbivore can change and this indirectly influences the development of the immature parasitoid (Godfray, 1994). Plants also affect the level of parasitation of the herbivore due to food plant quality (Price et al., 1980, Fox et al., 1996). And plants can provide shelter for the herbivore and thus reduce the efficiency of natural enemies (Sznajder and Harvey, 2003).

In order to investigate the effect of the host shift of DBM to peas on parasitoids, two parasitoid species were selected: Diadegma mollipla (Holmgren, 1868) (Hymenoptera: Ichneumonidae) the most important solitary endoparasitoid of DBM in Kenya and Diadegma semiclausum (Hellen), an exotic parasitoid that has been introduced to Kenya 
as part of a biocontrol programme for DBM. The local D. mollipla is considered a relative generalist. This species is also known as a parasitoid of the potato tuber moth, Phthorimaea operculella (Zeller) (Gelechiidae) and is reported to be indigenous to eastern and southern Africa. The original host is however unknown (Broodryk, 1971, Gupta, 1974, Azidah et al., 2000). Diadegma semiclausum has been widely used for biological control of DBM in various countries (Talekar and Shelton, 1993)and is known as a DBM specialist (Abbas, 1988).

On snowpeas fitness, parasitation and host location behaviour of the two species were investigated individually in the laboratory. Additionally interspecific competition between both species was tested. The influence of DBM on peas on the entire parasitoid complex associated with DBM was investigated under natural conditions in the field. 


\section{CHAPTER 2}

\section{Host shift to peas in the diamondback moth Plutella xylostella $\mathrm{L}$. and response of its parasitoid Diadegma mollipla}

\subsection{Abstract}

Host shifts in herbivorous insects are often thought to provide enemy-free space on the novel host plant. A population of the diamondback moth Plutella xylostella (L.) (Lepidoptera: Plutellidae) (DBM), known as an oligophagous pest on crucifers recently shifted to sugar snap- and snowpeas (Pisum sativum) in Kenya. As a result heavy damage was caused to these crops. The impact of this host shift on the interaction with Diadegma mollipla (Holmgren), one of the most frequent parasitoid species attacking DBM in this area was investigated. Parasitation rates and development of two strains of D. mollipla, reared on a DBM cabbage feeding strain and on the new DBM pea feeding strain, were dependent on the host-plant complex. Both parasitoid strains proved to be more effective on the novel host. Parasitation of the cabbage-D. mollipla strain on peas offered alone was four times higher than on cabbage also offered alone. However, when both crops were offered together, the level of parasitation dropped to the level of cabbage offered alone. D. mollipla developed equally well on both hosts, but cabbageD. mollipla had a longer development period. However, DBM cabbage pupae were significantly heavier than pea pupae and parasitation had no influence on these differences. Our research showed that D. mollipla prefers to parasitize the pea feeding DBM. The host shift of DBM to the novel host plant did not provide an enemy-free space as regards to the impact of the parasitoid species tested. The implications of these findings for the host-parasitoid relationship are discussed.

key words: Plutella xylostella, DBM, crucifers, host shift, enemy-free space, Diadegma mollipla, Pisum sativum 


\subsection{Introduction}

Although most herbivorous insect species are specialized feeders on just one plant family (Bernays and Chapman 1994), a few abrupt host shifts to new plant families have been reported (Strong, 1979, Bush 1994). A specialisation on specific plant species has been discussed in relation to the problem of sequestration of the often deleterious chemistry of the host plants for the herbivores (Ehrlich and Raven 1964). Another paradigm however relates the restricted diet breadth of herbivores to the action of natural enemies (Jeffries and Lawton 1984). Plants strongly influence the evolutionary and behavioral ecology of host-parasitoid associations. Plant species and structure influence the risk and level of parasitation (Price et al., 1980, Godfray, 1994). Stimuli emanating from the plant or the plant-herbivore interaction are used for host location (Vinson, 1976, Vet and Dicke, 1992, DeMoraes et al., 1998). Feeding on a novel plant species could be advantageous for the herbivore insect when specialised natural enemies (e. g. parasitoids) do not exploit their hosts on these novel plants.

The Diamondback Moth (DBM), Plutella xylostella (L.) (Lepidoptera: Plutellidae) is known as one of the most destructive pests on crucifers worldwide. It is considered stenophagous and limited to crucifers (Talekar and Shelton, 1993), although records of sporadic occurrences of DBM on other crops exist (Löhr, 2001). Recently a diamondback moth population in central Kenya, in the area of Lake Naivasha, shifted to pea, causing heavy damage to this plant (Löhr, 2001). We hypothesize that this novel host plant association will influence the interactions between the herbivore and its natural enemies.

One of the most frequent parasitoids of DBM in Kenya is an internal larval parasitoid of the ichneumonid family. According to a recently published revision of the Diadegma species complex attacking DBM (Azidah et al., 2000), the species found in Kenya was identified as D. mollipla (Holmgren, 1868). This species is also known as a parasitoid of the potato tuber moth, Phthorimaea operculella (Zeller) (Gelechiidae) and is reported to be indigenous to eastern and southern Africa. The original host is however unknown (Broodryk, 1971, Gupta, 1974, Azidah et al., 2000). In the east African highland D. 
mollipla is frequently found on DBM on brassica crops; however, parasitation rates are not particulary high. Overall field parasitation was reported to be less than $20 \%$ with $D$. mollipla being the most abundant species (Odour et al., 1996). Very little is known about the biology of this parasitoid species in association with the diamondback moth. In this study the effect of a host shift of the diamondback moth to peas on the parasitation and the development of Diadegma mollipla was investigated.

\subsection{Materials and Methods}

\subsubsection{Insect cultures of P. xylostella and D. mollipla}

Cabbage strain of P. xylostella (c-DBM). Diamondback moth larvae were collected in cabbage fields in Limuru in the Kiambu District, Kenya and reared in the laboratory (Temp. $=23 \pm 2^{\circ} \mathrm{C}$ ) on potted cabbage plants (Brassica oleracea L. var. capitata (Copenhagen Market)). Pupae were removed from the plants and after emergence transferred to a perspex cage $(43 \times 23 \times 22 \mathrm{~cm})$. As a stimulus for oviposition aluminium foil strips, coated with cabbage leaf extract, were fixed at the top of the cage. The foil was slightly crumpled in order to produce an irregular surface as tactile stimulus for the female moths for egg deposition. As food source cotton wool soaked with a $10 \%$ sugar solution was placed in a small plastic tube fixed to the cage wall. A water source was provided in the same way.

Pea strain of P. xylostella (p-DBM). Larvae were collected in a sugar snap pea field (Pisum sativum, var. Oregon sugar pod) near Naivasha in the Nakuru District south of Lake Naivasha, Kenya. These were subsequently maintained on potted plants of the same variety in the laboratory (Temp. $\left.=23 \pm 2^{\circ} \mathrm{C}\right)$. Pea leaf extract on aluminium foil was proved not to be a sufficient stimulus for oviposition. Instead of laying most of their eggs on the foil the female moths distributed them on the cage walls. Therefore a transparent crumpled plastic bag, containing a cabbage leaf was suspended from the top of a perpex cage and used for oviposition. So deposited eggs did not come into contact with the leaf and hatching larvae could be transfered from the bag to pea plants. 
Cabbage strain of D. mollipla (c-D. mollipla). Parasitized pupae from field collections carried out in cabbage fields in Kabsabet in the Nandi District, of western Kenya and in Limuru in the Kiambu District, central Kenya were collected to establish cultures for the experiments. Parasitoids were reared on second and third instar DBM larvae on cabbage in perpex cages. Parasitized DBM larvae were maintained separately. Pupae were collected into a vial and newly emerged parasitoid adults were then kept separately for at least one day to ensure mating. DBM larvae were renewed every 2 to 3 days until the parasitoids died. Honey solution (20\%) and water were supplied as described for the DBM culture.

Pea strain of D. mollipla (p-D. mollipla). The culture was established starting with a single pair found in DBM larvae collected from pea fields at Naivasha and maintained on a pea DBM culture. The maintenance of this culture followed the same procedure as for c-D. mollipla.

\subsubsection{Effect of host larvae on parasitation}

In order to compare the acceptance and parasitation rate of the two parasitoid strains on the common c-DBM and on the new p-DBM larvae, the following set-up was used. All experiments were conducted under laboratory conditions $\left(\mathrm{T}=23 \pm 2^{\circ} \mathrm{C}\right)$. Single mated 2-3 day old female $D$. mollipla were tested. Preliminary tests with $D$. mollipla showed peak searching activity after this period. A single cabbage leaf was infested with 25 second instar c-DBM larvae, four days old, and were kept in a small plastic container $(5 \times 8 \times 17 \mathrm{~cm})$. After two hours a female parasitoid was released into the container and left there for parasitation for $24 \mathrm{~h}$. Respectively, a pea leaflet of the same size as the cabbage leaf was infested with p-DBM larvae and treated as described above. Fully expanded leaves from four to six week old plants of both plant species were used. This experiment was carried out with both, c-D. mollipla on c-DBM and on p-DBM, p- $D$. mollipla on c-DBM and p-DBM. Each combination was replicated 20 times, except for the pea strain of the parasitoid. Here the number of replicates was lower (7 and 5). Unfortunately the culture collapsed during experimentation and could not be renewed 
because the species were not found again in the field.

After removing the parasitoid the DBM larvae were fed on their respective food plants. until reaching adult stage. The number of parasitoid pupae in each container was recorded. DBM larvae that died were dissected in order to search for the parasitoid egg or larvae to obtain the total number of parasitized DBM larvae. The single egg layed by D. mollipla was very easy to detect.

In this experimental setup naive and experienced parasitoids were tested. Naive females did not have contact with any DBM larvae before the experiment. To gain experience females were allowed to parasitize larvae of the DBM strain they emerged from $24 \mathrm{~h}$ before the trial.

\subsubsection{Effect of host plants on parasitation}

After having found successful parasitation of D. mollipla on the new DBM strain in the small containers, a larger set-up was installed in order to determine the influence of the host plant on the parasitation rate.

Tests described in this chapter were conducted with whole potted plants in a screened metal-framed cage measuring $60 \times 45 \times 45 \mathrm{~cm}$. Only the experienced c-D. mollipla strain was used, because naïve parasitoids tended to showing no searching behavior at all. The pea strain of the parasitoid was not available due to the above mentioned collapse of the culture. To reduce the influence of variability of performance for individual females three parasitoids were released in the cage. All treatments were replicated three times. 


\subsubsection{Single host plant and mixed host plant exposure.}

Four cabbage plants (4-6 weeks after transplanting; 6-8 leaves) were placed in the cage approximately $20 \mathrm{~cm}$ apart. Each plant was infested a day before exposure with 10 second instar c-DBM larvae. They were then exposed for $48 \mathrm{~h}$ to three to four day old parasitoids. The larvae were subsequently kept in plastic containers on cabbage leaves. Larvae of the same plant were kept together. The number of parasitized pupae was recorded. A similar experiment was conducted with p-DBM larvae on four pea plants offered as single host and in a mixed host plant situation with two pea and two cabbage plants with their respective DBM larvae.

\subsubsection{Host/parasitoid development}

In order to detect the development success of the two D. mollipla strains on c-DBM and p-DBM the parameters pupal weight and duration of development of parasitoids and hosts, respectively, were compared. Host survival was determined but due to a microsporidia infection in the c-DBM colony mortality caused by the parasitoid could not be distinguished from mortality caused by the infection of the larvae. Therefore data had to be dismissed. In small containers $(5 \times 8 \times 17 \mathrm{~cm}) 20$ to 30 DBM larvae were exposed for 24h to 3-4 females of D. mollipla. Age of larvae (4 days old), leaves of food plants and parasitoid/DBM combinations did not differ from the experimental setup previously described. The number of females was usually sufficient to parasitize most of the DBM larvae due to the small size of the container. After exposure, larvae were kept individually in vials on leaves or leaf discs of their respective food plants and checked daily for pupation. Spinning of the cocoon was considered as an indicator in order to distinguish between the larval and pupal period of the parasitoid. Pupae were weighed on a Mettler analytical balance to the nearest $0.01 \mathrm{mg}$. 


\subsubsection{Statistical analysis}

Multiple comparisons of mean development times and pupal weights of parasitoids were performed using an ANOVA (Student Newman Keuls Test). For the mean comparison of development and weight of unparasitized larvae of the two DBM strains the T-Test was used. Mean comparisons of parasitation rates between parasitoid/host combinations were conducted with the Student Newman Keuls Test and for the effect of host plants on parasitation rates the Tukey Test was performed. The 0.05 level was set for all comparisons made. The Chi-square adaptation test was used in order to determine if the sex ratio deviates from an expected 1:1 ratio. For the comparison of differences in sex ratios between parasitoid/DBM combinations the Chi-square $2 \times 2$ was applied. 


\subsection{Results}

\subsubsection{Effect of host larvae on parasitation}

Fig. 2.1 shows the parasitation rates of $D$. mollipla on the 2 DBM strains. The new peaDBM strain was accepted as host by both $D$. mollipla strains tested. The $\mathrm{p}-D$. mollipla strain parasitized on c-DBM as well as on p-DBM. In general individual parasitation varied considerably within the two D. mollipla strains. Average parasitation per naive female (A) was lowest for c-D. mollipla on c-DBM with $16.9 \%$, on p-DBM it was higher with $22.9 \%$. The highest mean number of parasitized larvae was found for $\mathrm{p}-D$. mollipla on p-DBM with $32.2 \%$. Despite the high variation among naïve females a tendency towards higher parasitation on p-DBM is found, although the differences between the mean number of parasitized larvae is not significant (Student Newman Keuls Test $\mathrm{df}=2,47 ; \mathrm{F}=1.08 ; \mathrm{p}=0.348$ n.s.). In experienced parasitoids a difference can be clearly demonstrated (B). The mean parasitiation of on cabbage experienced c- $D$. mollipla was with $47.5 \%$ significantly higher on p-DBM than on c-DBM with $23.6 \%$.
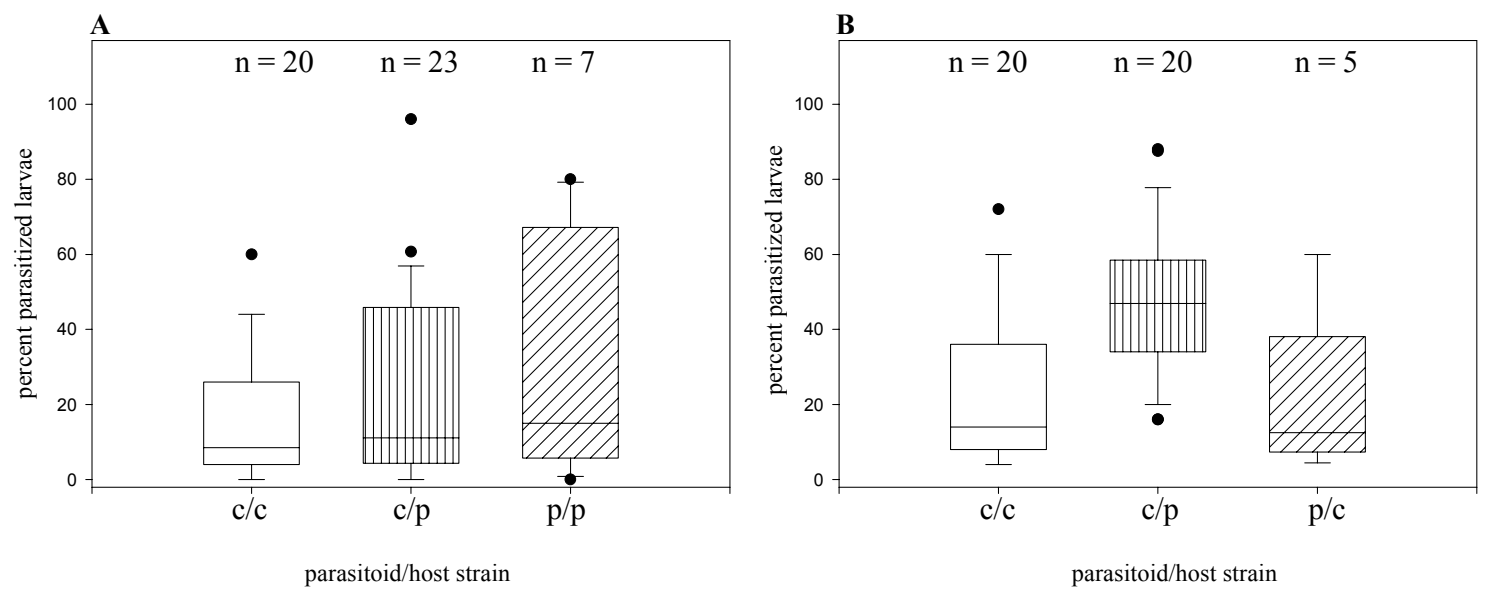

Fig. 2.1: Variability of parasitation of individual naive (A) and experienced (B) Diadegma mollipla: c/c (c-D. mollipla on c-DBM), c/p (c-D. mollipla on $\mathrm{p}-\mathrm{DBM}), \mathrm{p} / \mathrm{p}$ ( $\mathrm{p}-D$. mollipla on $\mathrm{p}-\mathrm{DBM}), \mathrm{p} / \mathrm{c}$ ( $\mathrm{p}-D$. mollipla on $\mathrm{c}-\mathrm{DBM})$. The on pea experienced pea strain showed with $23.4 \%$ a mean parasitation similar to the cabbage strain (Student Newman Keuls Test; $\mathrm{df}=2,42 ; \mathrm{F}=7.27 ; \mathrm{p}<0.05)$. Missing data and low number of females tested in $\mathrm{p}-D$. mollipla was due to the already mentioned collapse of this culture. 


\subsubsection{Effect of host plants on parasitation}

The host plant complex had a strong influence on the parasitation rate (Fig. 2.2). The parasitation rate of c- $D$. mollipla on cabbage plants alone was with $6.1 \%$ significantly lower than on DBM exposed on pea alone with $26.5 \%$. When both host plants were offered simultaneously parasitation was comparable to cabbage offered alone $(3.5 \%)$ (Tukey's Test; $\mathrm{df}=8,27 ; \mathrm{F}=4.54 ; \mathrm{p}<0.05$ ). In the graph significant differences in parasitation are indicated by different capital letters. In the mixed exposure however, a higher proportion of larvae was parasitized on peas $(2.6 \%)$ than on cabbage $(0.9 \%)$, showing again clearly the preference for the pea-DBM complex.

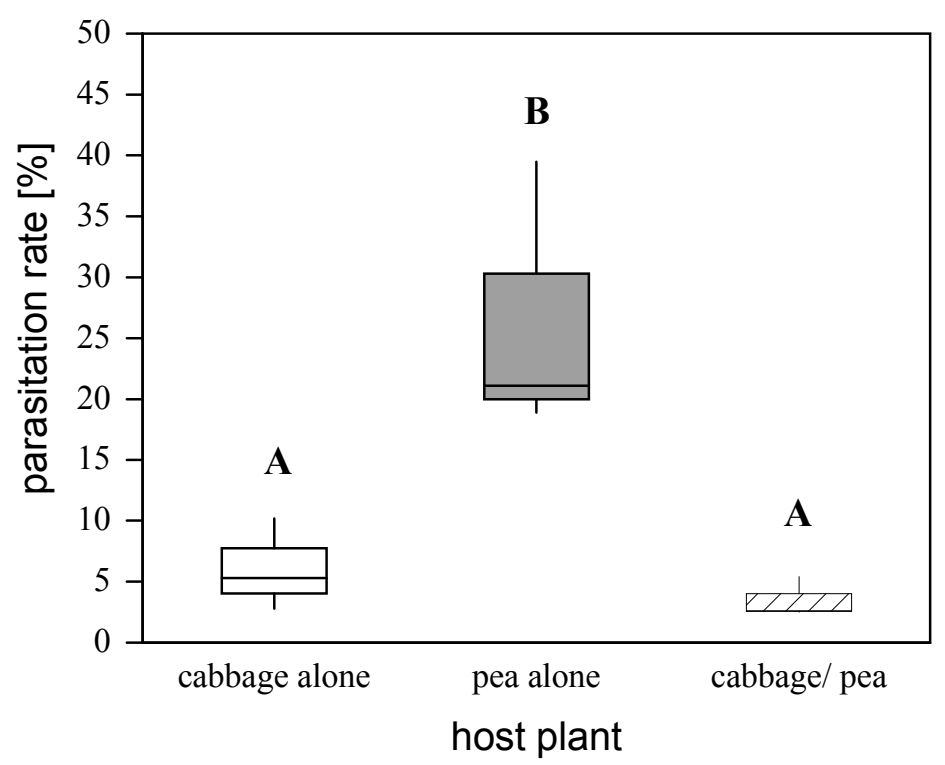

Fig. 2.2: Influence of host plants on parasitation of c-Diadegma mollipla. Means from 3 replicates.

\subsubsection{Development of P. xylostella and D. mollipla}

In Table 2.1 the development time of unparasitized DBM, both cabbage and pea strain, are given in the first 2 lines. In the following lines the development duration of the parasitoid/host combinations are shown. DBM larvae were exactly 4 days old when 
exposed to the parasitoids. In order to compare the development time of parasitized with unparasitized DBM development duration was counted from day 4 (exposure day). Spinning of the cocoon was considered as an indicator in order to distinguish larval and pupal duration. Thus the development of four day old p-DBM larvae was with 12.2 days significantly slower as compared to c-DBM (10.7 days) (T-test; $\mathrm{df}=160,137 ; \mathrm{F}=1.85$; $\mathrm{p}<0.001$ ), indicated with $* * *$ in Table 2.1. This was a result of the longer pupation period required by the p-DBM (6.5 days) as compared to 5.4 days for the c-DBM (Ttest; $\mathrm{df}=160,137 ; \mathrm{F}=5.47 ; \mathrm{p}<0.01)$. Larval duration was similar in both DBM strains (5.3 and 5.7 days) (T-test; $d f=160,137 ; F=1.25 ; p=0.175)$.

Table 2.1: Comparison of development time of Diadegma mollipla reared on Plutella xylostella on cabbage or peas. To distinguish between larval and pupal duration the spinning of the cocoon by the DBM larvae was considered. Larval duration and development time of unparasitized DBM larvae were counted from day of exposure to parasitoid (4 days after hatching).

\begin{tabular}{llcccc}
\hline $\begin{array}{c}\text { parasitoid } \\
\text { strain }\end{array}$ & host strain & $\begin{array}{c}\text { development } \\
\text { time (total) } \\
\text { [days] }\end{array}$ & $\begin{array}{c}\text { larval duration } \\
\text { [days] }\end{array}$ & $\begin{array}{c}\text { pupal duration } \\
\text { [days] }\end{array}$ & $\begin{array}{c}\text { SNK } \\
\text { group }\end{array}$ \\
\hline- & cabbage & $10.7 \pm 0.9^{* * *}$ & $5.3 \pm 0.7$ & $5.4 \pm 0.6^{* * *}$ & $\mathrm{~A}$ \\
- & pea & $12.2 \pm 1.3^{* * *}$ & $5.7 \pm 0.8$ & $6.5 \pm 1.4^{* * *}$ & $\mathrm{~A}$ \\
cabbage & cabbage & $15.8 \pm 0.7$ & $6.3 \pm 0.6$ & $9.5 \pm 0.7$ & $\mathrm{~B}$ \\
pea & cabbage & $15.5 \pm 0.5$ & $6.0 \pm 0.6$ & $9.5 \pm 0.5$ & $\mathrm{~B}$ \\
pea & pea & $15.7 \pm 0.9$ & $6.5 \pm 1.0$ & $9.2 \pm 1.1$ & $\mathrm{~B}$ \\
cabbage & pea & $16.9 \pm 1.5$ & $8.6 \pm 0.9$ & $8.3 \pm 1.2$ & $\mathrm{C}$ \\
\hline
\end{tabular}

The parasitoid developed successfully on both host strains and showed similar development time except the cabbage strain of the parasitoid on pea-DBM (Table 2.1). Development was considerably longer compared to unparasitized DBM because the parasitoid had a longer pupal period. Significant differences in means are indicated with capital letters in Table 2.1. Pupal duration was with 8.3 days shorter for c-D. mollipla on p-DBM than in the other combinations with 9.2 and 9.5 days, respectively (Student Newman Keuls Test; $\mathrm{df}=3,215 ; \mathrm{F}=18.4 ; \mathrm{p}<0.001)$. Compared to unparasitized DBM 
larvae larval duration of the parasitized larvae was extended in both host strains (Table 2.1). For three parasitoid/DBM combinations the larval period was similar and ranged between 6.0 days for $\mathrm{p}-D$. mollipla on c-DBM and 6.5 days for $\mathrm{p}-D$. mollipla on $\mathrm{p}$ DBM and c-D. mollipla on c-DBM ranging in between the two former combinations (6.3 days). The slowest larval duration was determined for c-D. mollipla on p-DBM (8.6 days) (Student Newman Keuls Test; $d f=3,269 ; F=18.5 ; p<0.001$ ).

Pupal weight of unparasitized p-DBM (4.5 mg) was significantly lower than for c-DBM (5.4 mg) (Table 2.2). Correspondingly, pupal weights of parasitoids reared from p-DBM were lower (4.4mg and $4.2 \mathrm{mg}$, cabbage and pea strain, respectively) than from c-DBM (5.3mg and $5.7 \mathrm{mg}$ cabbage and pea strain, respectively). There was no significant difference between the weight of parasitized and unparasitized pupae and similar weights were grouped according to their host plants in Table 2.2 (Student Newman Keuls Test; $\mathrm{df}=5,588 ; \mathrm{F}=62.74 ; \mathrm{p}<0.001)$. The sex ratio of emerging parasitoid adults was slightly male-biased for the c-parasitoid strain on both DBM strains and for p- $D$. mollipla raised on $\mathrm{p}-\mathrm{DBM}$. The ratio for $\mathrm{p}-D$. mollipla reared on c-DBM was femalebiased. This ratio was significantly biased for c-D. mollipla on $\mathrm{p}-\mathrm{DBM}$; in the other parasitoid/host combinations the sex ratio did not significantly deviate from expectation. 
Table 2.2: Comparison of pupal weight of Diadegma mollipla reared on Plutella xylostella on cabbage or on peas with unparasitized diamondback moth larvae. Exposure of 4 day old DBM larvae (L2).

\begin{tabular}{llccc}
\hline $\begin{array}{l}\text { parasitoid } \\
\text { strain }\end{array}$ & host strain & pupal weight $[\mathbf{m g}]$ & $\begin{array}{c}\text { SNK } \\
\text { group }\end{array}$ & $\begin{array}{c}\text { sex ratio } \\
\text { females }\end{array}$ \\
\hline- & cabbage & $5.4 \pm 0.7$ & $\mathrm{~A}$ & - \\
cabbage & cabbage & $5.3 \pm 0.8$ & $\mathrm{~A}$ & 0.75 \\
pea & cabbage & $5.7 \pm 0.8$ & $\mathrm{~B}$ & 1.60 \\
- & pea & $4.5 \pm 0.7$ & $\mathrm{C}$ & - \\
cabbage & pea & $4.4 \pm 0.5$ & $\mathrm{C}$ & $0.73 *$ \\
pea & pea & $4.2 \pm 0.7$ & $\mathrm{C}$ & 0.80 \\
\hline
\end{tabular}




\subsection{Discussion}

In our experiments overall parasitation was low in all combinations investigated and performance of individual females was very variable, independent of their hosts. Experienced females were more effective than naive ones and reached higher parasitation rates. Despite individual variability preference experiments revealed significantly higher parasitation on p-DBM as compared to c-DBM.

Two hypotheses may be discussed with regard to these observations: 1. D. mollipla has only developed a loose association with DBM and its host plant. 2. There must be a factor that renders DBM on crucifers less attractive than on peas. The first hypothesis is supported by Broodryk (1971) who lists D. mollipla as an important parasitoid of the potato tuber moth (PTM) on potato and tobacco in southern Africa and on potato in Yemen (Kroschel, 1993). However, as PTM is an introduced species to Africa and D. mollipla seems to be indigenous, PTM cannot be the original host of this species. It is therefore reasonable to assume that D. mollipla is a parasitoid with a considerable host plasticity. We assume that it might be found to parasitize more free-living or leaf mining species of microlepidoptera. A lack of intrinsic cues to find the host plant of DBM may also explain the generally low parasitation rates of DBM observed in the laboratory (Akol, 2003) and thus its irrelevance for the control of DBM field populations (Oduor et al. 1996, Löhr, unpublished survey data). As for the second hypothesis, concerning factors for the higher attractivity of peas, a few published papers report an influence of host plants on parasitation levels on DBM. Beck and Cameron (1990) related different levels of parasitation by D. semiclausum and Diadromus collaris on three vegetable brassicas to the accessibility of the host larvae for the parasitoids. Broccoli, showing highest parasitation, does not form heads like cabbage, thus the leaves do provide less shelter for the larvae as compared to leaves tightly attached to the head. Idris and Grafius (1996) reported a higher percentage of parasitation of Diadegma insulare on DBM on cultivated brassicas than on wild Brassicaceae. Their findings may be explained by plant quality, D. insulare parasitizing more DBM larvae on N-fertilized than on unfertilized plants (Fox et al., 1990, 1996). However, differences in accessibility of DBM do not explain the significant preference 
of D. mollipla females for DBM feeding on peas, because DBM larvae were freely exposed on leaves during the experiments. At this point we are not able to rule out the possibility of differences in DBM suitability as hosts mediated by plant quality. Although fertilizer treatments did not differ between the two host plant species, we do not have data on specific plant compounds rendering the plants more or less attractive to D. mollipla.

However, the preference of D. mollipla for DBM feeding on peas may be explained by cues used for host location. It is now well documented that hymenopterous parasitoids use infochemicals to locate their hosts (e.g. reviewed in Vet and Dicke, 1992). These volatiles may originate from the host plant, the herbivore itself or the interaction between plant and herbivore (Drost et al., 1986, Turlings et al., 1991, Agelopoulos and Keller, 1994, Zaki et al., 1998). Evidence that a DBM parasitoid is attracted by kairomones was found in D. semiclausum (Davis, 1987). Residence and searching time increased when parasitoid females were offered leaves with hosts and silk as compared to silk or clean leaves.

One-to-one transplantation of these results to the given plant-host-parasitoid system is however premature, because cues used can differ even within a genus. Shiojiri et al. (2000) demonstrated that Cotesia plutellae and C. glomerata, both parasitoids of Pieris rapae, showed different preferences in flight response experiments towards the plantherbivore complex. Oviposition attempts into feeding holes of both plants tested were observed, even when larvae were absent. However, although plant injury derived volatiles in combination with specific volatiles emitted by the host plant could be an important cue in the searching behaviour of $D$. mollipla this still does not explain the preference in c-DBM experienced females of the $\mathrm{c}-D$. mollipla strain for $\mathrm{p}$-DBM larvae. Parasitoids associated with crucifer specialist herbivores were shown to be attracted by volatile isothiocyanates (mustard oils) typically released by crucifers when injured (Pivnick, 1993, Murchie, et al., 1997). For D. mollipla crucifer volatiles are unlikely to be used for host location. In Chapter 3 it is demonstrated that D. mollipla females are attracted to odours emitted by peas infested with but not to odours emitted by cabbage plants infested with DBM larvae. This is because D. mollipla is either attracted to a 
non-specific blend of volatiles from a variety of host-infested plants, or it uses other shared cues present in several hosts or host-plant complexes. Therefore we hypothesize that DBM-crucifer complex is not the preferred host-plant association of D. mollipla, although it is widely accepted because of its high abundance and therefore easy accessibility in Kenya.

D. mollipla was able to develop in either DBM strains without visible problems. Only development time and pupal weight are affected, probably due to growth differences of DBM on the two host plants or of differing secondary compounds ingested by the larvae.

Koinobiont parasitoids rely on their host's nutrition and growth potential (Mackauer et al., 1997). When feeding on different food plants, physiology and size of the herbivore can change and this influences the development of the immature parasitoid indirectly (Godfray, 1994). For example Idris and Grafius (1996) found that developmental time of Diadegma insulare on DBM on wild crucifers was longer than on cultivated brassica crops. Larval duration of larvae parasitized by D. mollipla was longer on both plants as compared to unparasitized larvae. Nutritional demands of the parasitoid often reduce growth and behavior of their hosts (Strand et al., 1988, Harvey et al., 1999). In contrast Yang et al. (1994) found no difference in duration of larval instars between parasitized and healthy DBM larvae, but food consumption was reduced in larvae parasitized by Diadegma semiclausum.

Growth of either parasitized and unparasitized DBM was dependent on the food plant. Pupae of DBM were heavier on cabbage than on pea. Either a lower nutritional value or lacking feeding stimulants of peas could be the reason for ths incident. 


\section{CHAPTER 3}

\section{Generalism versus specialism: Responses of Diadegma mollipla (Holmgren) and Diadegma semiclausum (Hellen), to the host shift of the diamondback moth (Plutella xylostella $\mathrm{L}$.) to peas}

\subsection{Abstract}

A population of the diamondback moth Plutella xylostella (L.) (Lepidoptera: Plutellidae) (DBM), known to be an oligophagous pest on crucifers, was recently found to infest sugar snap- and snowpeas in the Rift Valley in Kenya, causing heavy damage. The influence of this host shift on host location preferences of two parasitoids was investigated: The indigenous Diadegma mollipla (Holmgren) regarded as a relative generalist, because of rather low parasitation $(<15 \%)$ on crucifers and Diadegma semiclausum (Hellen), regarded as highly specific to DBM. In this study, the attractiveness of different odour sources was compared for the two parasitoid species. The bioassay tests were conducted in a Y-tube olfactometer, testing cabbage and pea related odours (larvae, faeces and DBM infested plants) either against clean air or against each other. Females were released individually for five minutes into the stem of the olfactometer and their first choice and the time spent in each arm were recorded. $D$. mollipla was not significantly attracted to any cabbage related odours but showed a significant preference for the DBM infested pea plant when tested against clean air. $D$. semiclausum was highly attracted to the undamaged cabbage plant and odours related to cabbage. On the other hand, peas infested with DBM, showed no attractiveness to this parasitoid. The results showed that specialisation of D. semiclausum is mediated by host plant signals, associated with crucifers, which are not encountered in DBM feeding on peas. Whereas for D. mollipla, although a frequent parasitoid on DBM in crucifers, volatiles emitted by these plants might not be used as primary cues for host location. This species may respond largely to chemicals yet unknown and associated with a variety of plant-herbivore interactions.

Keywords: parasitoids, host location, infochemicals, $P$. xylostella, Pisum sativum, 


\subsection{Introduction}

Chemical cues play an important role for the foraging behaviour and decision-making of parasitoids (e.g. Vinson 1976, Dicke and van Loon, 2000). Specifically, plant volatiles often guide parasitoids to their host habitats, but volatiles used by natural enemies may also originate from herbivores, symbionts associated with herbivores, and interactions between plants and these other sources (Bottrell et al., 1998). For many parasitoids the plant is a key factor for host location (Dicke, 1994, Geervliet et al., 1994, Ngi-Song et al. 1996, 2000). Specialists are expected to show more restricted preferences for plant-host complexes through responses to specific cues resulting from the interaction between a host and its food plant. On the other hand, generalist parasitoids are assumed not to rely on specific cues because of the great diversity of volatiles emanating from different hosts and plants (Vet and Dicke, 1992). Therefore, the shift of a herbivore to a new food plant should have different effects on different members of its parasitoid community. It should result in a loss of specific, plant-related cues and, therefore, to a reduced searching efficiency, especially for the specialist. This is of importance as parasitoids often serve as biological control agents and their effectiveness in regulating an insect pest largely depends on their host location behaviour. For the generalist, plant-related cues might play a lesser role and are, therefore, not as important for searching efficiency. But this would depend on the type of cues the generalist uses. To test this hypothesis, the system Plutella xylostella L. and its parasitoids, Diadegma mollipla and D. semiclausum, was studied.

In the Rift Valley in Kenya, the diamondback moth (DBM) Plutella xylostella L. (Lepidoptera: Plutellidae) has broadened its diet by adding snowpeas (Pisum sativum L.) to their normal host range, usually restricted to crucifers (Löhr, 2001). The diamondback moth is a very destructive pest on crucifers and difficult to control, since it has acquired resistance to all major pesticides (Talekar and Shelton, 1993). Introduction of parasitoids constitutes an important biological control method for this species. In Kenya, Diadegma semiclausum (Hellen) (Hymenoptera: Ichneumonidae), has recently been introduced to reduce DBM populations in crucifers. As for many important parasitoids, there is a lack of information about its host finding behaviour and 
mediating cues (Verkerk and Wright 1996). Despite its importance for the biological control of DBM, its host location behaviours has not been studied intensely. The most recent work that has been done so far is by Ohara et al. (2003) who demonstrated that the host plant plays a role in the host finding behaviour of D. semiclausum. In this study, we consider D. semiclausum as a specialist parasitoid as suggested by Wang and Keller (2002). According to the literature, DBM is the only known host and therefore crucifers the main host plant (Abbas, 1988).

The second parasitoid we studied is Diadegma mollipla (Holmgren) (Hymenoptera: Ichneumonidae). The cues deployed for host finding by this species are unknown. It is reported to be indigenous to eastern and southern Africa (Azidah et al., 2000). It is frequently found on DBM in Kenya, but low parasitation rates suggest that it has a broader host range. Except for the potato tuber moth, Phthorimaea operculella (Zeller) (Lepidoptera: Gelechiidae), no other host is yet known (Broodryk, 1971, Gupta, 1974). In this study, we treat the species as a generalist.

The attractiveness of plant-related cues for D. mollipla and D. semiclausum was tested in order to determine the effect of the host shift on both species. Parasitoids were given choices involving odours related to cabbage, the original host plant of DBM, and odours related to snowpeas, the new host plant.

\subsection{Material and Methods}

\subsubsection{Parasitoids}

Diadegma mollipla was obtained from cabbage fields at Wundanyi in Taita Taveta District of eastern Kenya and Maragua in Muranga District in central Kenya. Cultures were started in 2001 and frequently newly collected individuals from the field were added to the colonies. A colony of $D$. semiclausum was started in 2001 from pupae from a laborartory culture imported from the Asian Vegetable Research and Development Center (AVRDC) in Taiwan. Experiments were started a year after colonies had been established in the laboratory. Both parasitoid species were reared on second and third 
instar DBM larvae on cabbage in perpex cages. Parasitized larvae were fed on cabbage leaves in plastic boxes covered with a mesh to allow ventilation until pupation. Parasitoid pupae were collected from the cabbage leaves and kept in smaller clean plastic containers. Emerging adults were then released into an empty perpex cage $(20 \times 20 \times 25 \mathrm{~cm})$. Honey was provided as a food source. To ensure mating adults remained for at least 2 days in the cage. They did not have contact with plants or host larvae before the tests. Tested females were between 2 and 6 days old.

\subsubsection{Host larvae}

The cabbage strain of the diamondback moth originated from cabbage fields at Wundanyi and was reared in the laboratory $\left(\mathrm{T}=23 \pm 2^{\circ} \mathrm{C}\right)$ on potted cabbage plants (Brassica oleracea L. var. capitata (Copenhagen Market)). Diamondback moth larvae reared on cabbage are referred to as c-DBM.

Larvae of the DBM pea strain were collected from a sugar snap pea field (Pisum sativum, var. Oregon sugar pod) near Naivasha in Nakuru District, Kenya. Since 1999, the colony has been maintained on potted snowpeas (Pisum sativum, var. Oregon sugar pod). Diamondback moth larvae from peas are referred to as p-DBM.

Potato tuber moth larvae (PTM) were obtained from potato fields in Limuru in Kiambu District, central Kenya. They were not kept in culture, but collected from the field when needed.

\subsubsection{Host plants}

For tests involving cabbage-related odours, B. oleracea L. var. capitata (Copenhagen Market) was used, and for the snowpea-related odours, $P$. sativum, var. Oregon sugar pod was used. Potatoes (undefined variety) used were obtained from the local market. 


\subsubsection{Behaviour assays}

The olfactory attractiveness of DBM larvae, faeces and host plant with feeding larvae to D. mollipla and D. semiclausum females was studied in a Y-tube olfactometer (armlength $15 \mathrm{~cm}$, diameter $3.5 \mathrm{~cm}$ ). Tests were conducted in a bioassay room at a constant temperature of $23 \pm 2{ }^{\circ} \mathrm{C}$. Two neon tubes $(18 \mathrm{~W})$ provided a steady illumination of the test area. Individual odour sources were placed, according to their size, in a glass tube or a glass jar with an airtight lid connected to an olfactometer arm by a Teflon tube $(0.5 \mathrm{~cm}$ inner diameter). Air from an inlet pump (Air Cadet Vaccum Pressure Station from Cole \& Parmer) was passed through an activated charcoal filter for purification, then through a flow meter into two separate odour source tubes/jars. A second flow meter was connected between the stem of the olfactometer and a second pump, which exhausted air out of the system. For odour sources (faeces and larvae) in the glass tube, airflow into the olfactometer was set at $100 \mathrm{ml} / \mathrm{min}$ and at the exit at 220 $\mathrm{ml} / \mathrm{min}$, and for whole plants offered in glass jars they were set at $130 \mathrm{ml} / \mathrm{min}$ and $250 \mathrm{ml} / \mathrm{min}$ respectively.

Two to six day old mated female parasitoids without oviposition experience were introduced individually in the stem of the Y-olfactometer. They were allowed to stay for $5 \mathrm{~min}$. Parasitoids that crossed the line, $0.5 \mathrm{~cm}$ beyond the intersection, and remained in one arm for at least $20 \mathrm{sec}$ were recorded as having made a first choice. Total time spent in either one or both arms was recorded during the observation period. Females that remained in the stem or spent less than $20 \mathrm{sec}$ in one of the arms were recorded as showing no response. If they switched between the two arms without initially spending more than $20 \mathrm{sec}$ in one of the arms they were recorded as not having made a definite choice but total time spent was still measured. To avoid bias the odour sources were switched between the left and the right arm of the Y-tube after every 10 parasitoids. At least 60 parasitoids were tested for each treatment. 
The following odour source choices were tested for both parasitoid species:

choice

parasitoid

method

\begin{tabular}{ll}
\hline 1. c-DBM larvae/clean air & both \\
2. p-DBM larvae/clean air & D. semiclausum
\end{tabular}

Twenty $2^{\text {nd }}$ to $3^{\text {rd }}$ instar DBM larvae that fed on cabbage or pea respectively before the experiment were placed in one of the glass tubes $(2.5 \mathrm{~cm}$ in diameter, length $10 \mathrm{~cm})$. The other tube of the same size remained empty.

\begin{tabular}{ll}
\hline 3. c-DBM faeces/clean air & both \\
4. p-DBM faeces/clean air & D. mollipla
\end{tabular}

Fresh faeces produced by 30 DBM larvae (L2-L3) during $24 \mathrm{~h}$ was placed in the glass tube. Every $30 \mathrm{~min}$ the faeces was changed to ensure a consistent odour.

\begin{tabular}{lll}
\hline $\begin{array}{l}\text { 5. c-DBM infested cabbage } \\
\text { plant/clean air }\end{array}$ & both & In a glass jar (2l capacity) an entire \\
& & potted plant (4 weeks old $)$ with 20 \\
& feeding larvae was placed. The other \\
& glass jar of the same size remained \\
6. p-DBM infested pea & & empty. DBM larvae were allowed to feed \\
plant/clean air & for 20 hours before the experiment.
\end{tabular}

7. PTM infested potato/clean $\quad$ D. mollipla air

Two potatoes were infested with five $2^{\text {nd }}$ to $3^{\text {rd }}$ instar PTM larvae each. Larvae fed for 2 days in the tuber. The tubers were placed into a glass jar (0.51 capacity) and tested against an empty jar.

\begin{tabular}{cll}
\hline $\begin{array}{l}\text { 8. c-DBM infested cabbage } \\
\text { plant/p-DBM infested pea } \\
\text { plant }\end{array}$ & both & $\begin{array}{l}\text { In glass jars (21 capacity) entire potted } \\
\text { plants (4 weeks old) with 20 feeding } \\
\text { larvae were placed. DBM larvae were } \\
\text { allowed to feed for 20 hours before the } \\
\text { experiment. }\end{array}$ \\
$\begin{aligned} \text { 9. uninfested cabbage plant/c- } \\
\text { DBM infested pea plant }\end{aligned}$ & D. semiclausum \\
\hline $\begin{aligned} \text { 10. PTM infested potato/c- } \\
\text { DBM } \\
\text { infested } \\
\text { cabbage }\end{aligned}$ & D. mollipla & $\begin{array}{l}\text { Two potatoes infested with 5 PTM larvae } \\
\text { each and placed in a glass jar. Potted } \\
\text { cabbage and pea plants were infested } \\
\text { with 20 DBM larvae (see above). }\end{array}$ \\
$\begin{aligned} \text { 11. PTM infested potato/p- } \\
\text { DBM } \\
\text { infested pea }\end{aligned}$ & D. mollipla & \\
\hline
\end{tabular}




\subsubsection{Statistical analysis}

The results of the choice between odour sources were analysed with the Yates-corrected Chi-Square test. For differences in the time spent in the two indiviual arms the Tukey test was used after an arcsine transformation (GLM proc SAS Institute, 1990) . Parasitoids that made no choice were excluded from the analyses. Parasitoids that made no definite first choice but spent time in both olfactometer arms were also included in the GLM analyses.

\subsection{Results}

\subsubsection{Choice tests with clean air}

D. mollipla did not show preference for either c-DBM larvae, their faeces or DBMinfested cabbage plant when tested against clean air (Fig. 3.1A). The parasitoids were equally distributed and the females spent nearly the same amount of time in the two arms of the Y-tube (Fig. 3.1B). In contrast, D. semiclausum responded clearly to cabbage-related odour sources. The preference was strongest for larvae-infested cabbage plant $(81.6 \%$ of the choices made and $74.1 \%$ of the time spent in the treated arm) (Fig. 3.2). Even c-DBM faeces as well as larvae alone were significantly preferred over clean air, although larvae alone elicited the weakest response, which was only significant with respect to the time spent (Fig. 3.2B).

On the other hand, p-DBM faeces was not attractive to D. mollipla, but the parasitoid showed significant preference for the larvae-infested pea plant, although not very pronounced (60\% of first choice made and time spent) (Fig. 3.3). D. semiclausum was indifferent to larvae-infested pea plant (Fig. 3.4). Both, arm selection and time spent in the two arms was around 50\%. The p-DBM larvae alone were even less attractive than clean air with $37.7 \%$ and $42 \%$ for first choice and time spent respectively (Fig. 3.4).

Potatoes infested with PTM, the second known host of D. mollipla, showed a similar attractiveness to DBM-infested pea plant. D. mollipla spent significantly more time in 
the arm with the potato related odour $(58.7 \%)$ and females made $59.6 \%$ of the choices in favor of the odour (Fig. 3.3).
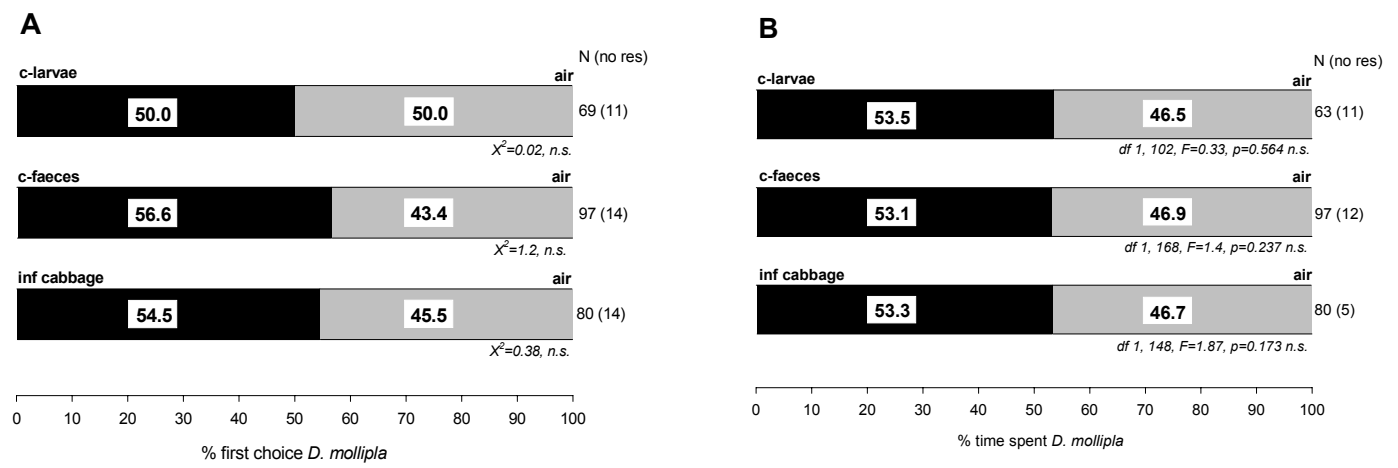

Fig. 3.1: Responses of naive Diadegma mollipla females to cabbage-related odours tested against clean air $(\mathrm{c}-\mathrm{DBM}$ larvae $=\mathrm{c}$-larvae, $\mathrm{c}-\mathrm{DBM}$ faeces $=\mathrm{c}$-faeces, c-DBMinfested cabbage plant $=$ inf cabbage). A) percentage of the number of first choices made. B) percentage time spent in the arms with respective odours within observation period of $5 \mathrm{~min}$. $\mathrm{N}=$ number of females. Number of parasitoids that did not respond are indicated in brackets. Asterisks indicate statistically significant preferences within tests (Yates corrected $\mathrm{Chi}^{2}-$ Test for first choice and Tukey Test for time spent) $\left({ }^{*} \mathrm{p}<0.05\right.$, $* * \mathrm{p}<0.01, \mathrm{n} . \mathrm{s} .=$ not significant).
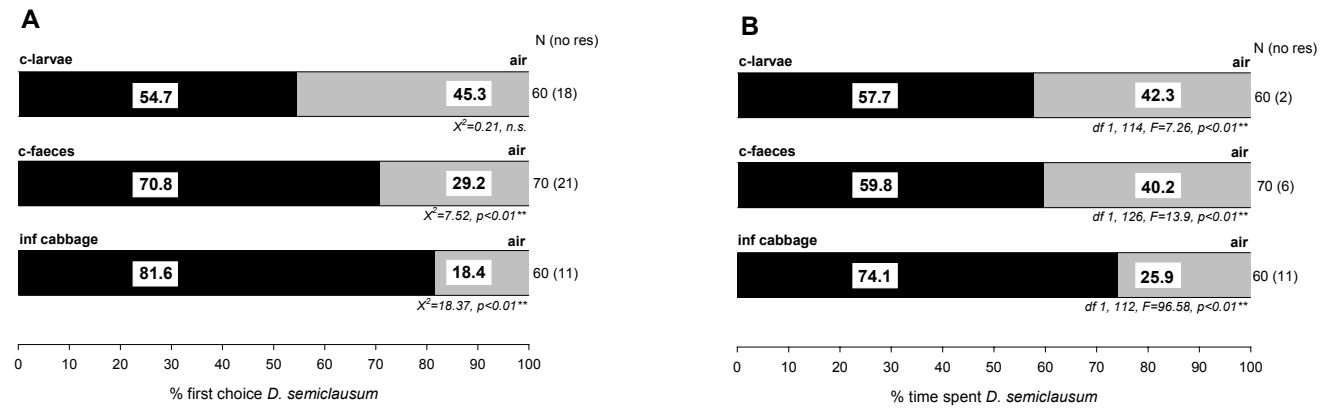

Fig. 3.2: Responses of naive Diadegma semiclausum females to cabbage-related odours tested against clean air $(\mathrm{c}-\mathrm{DBM}$ larvae $=\mathrm{c}$-larvae, $\mathrm{c}-\mathrm{DBM}$ faeces $=\mathrm{c}$-faeces, $\mathrm{c}$-DBMinfested cabbage plant $=$ inf cabbage). A) percentage of the number of first choices made. B) percentage time spent in the arms with respective odours within observation period of $5 \mathrm{~min} . \mathrm{N}=$ no. of females. No. of parasitoids that did not respond are indicated in brackets. Asterisks indicate statistically significant preferences within tests (Yates corr. Chi ${ }^{2}$-Test for first choice and Tukey Test for time spent) $\left({ }^{*} p<0.05,{ }^{*} p<0.01\right.$, n.s. $=$ not significant). 


\section{A}

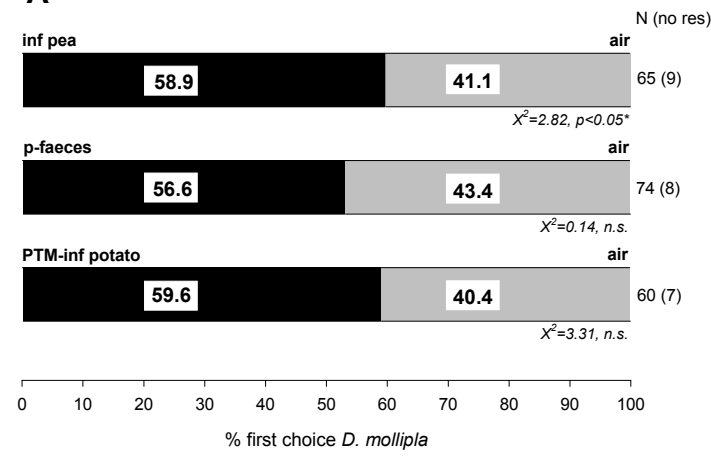

B
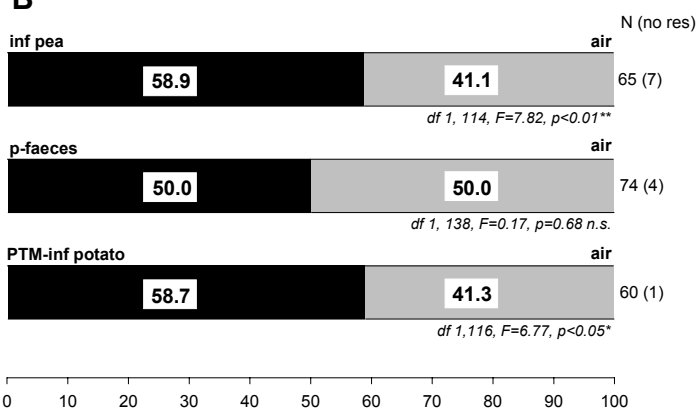

Fig. 3.3: Responses of naive Diadegma mollipla females to pea-related odours tested against clean air $(p-D B M$ faeces $=p$-faeces, $p$-DBM-infested pea plant $=$ inf pea). A) percentage of the number of first choices made. B) percentage time spent in the arms with respective odours within observation period of $5 \mathrm{~min}$. $\mathrm{N}=$ number of females. Number of parasitoids that did not respond are indicated in brackets. Asterisks indicate statistically significant preferences within tests (Yates corrected $\mathrm{Chi}^{2}$-Test for first choice and Tukey Test for time spent) $\left(* \mathrm{p}<0.05,{ }^{* *} \mathrm{p}<0.01, \mathrm{n} . \mathrm{s} .=\right.$ not significant $)$.

A

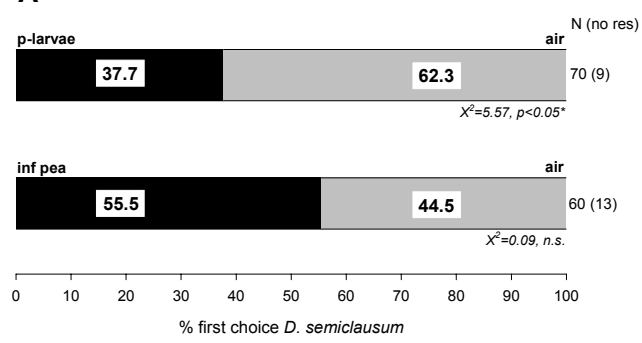

B
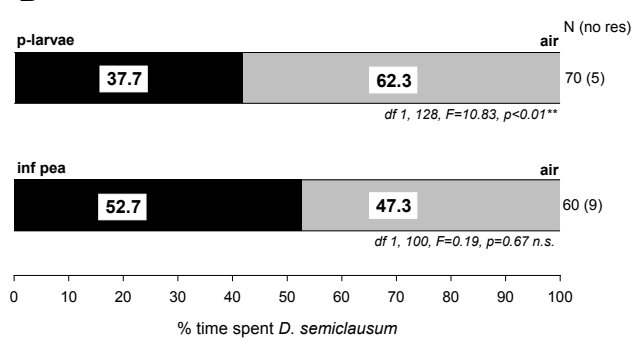

Fig. 3.4: Responses of naive Diadegma semiclausum females to pea-related odours tested against clean air ( $\mathrm{p}$-DBM larvae $=$-larvae, $\mathrm{p}$-DBM-infested pea plant $=$ inf pea). A) percentage of the number of first choices made. B) percentage time spent in the arms with respective odours within observation period of $5 \mathrm{~min}$. $\mathrm{N}=$ number of females. Number of parasitoids that did not respond are indicated in brackets. Asterisks indicate statistically significant preferences within tests (Yates corrected $\mathrm{Chi}^{2}$-Test for first choice and Tukey Test for time spent $)\left({ }^{*} \mathrm{p}<0.05,{ }^{*} \mathrm{p}<0.01, \mathrm{n} . \mathrm{s} .=\right.$ not significant $)$. 


\subsubsection{Choice tests with two odours}

Since the strongest responses of both parasitoids were elicited by larvae-infested plants (pea and potato for D. mollipla and cabbage for D. semiclausum), they were tested against each other.

D. mollipla females chose the larvae-infested pea plant slightly more often (55.5\%) than the infested cabbage plant, but did not spend more time in that arm (Fig. 3.5). However, these results are not significant and show that, given a choice between these two plants, there is no clear preference for one. When the DBM-infested cabbage was tested against PTM-infested potatos, D. mollipla was significantly more attracted to the infested potatos, with $61 \%$ of the females choosing the arm with the PTM-potato odour (Fig. 3.5). However, time spent in the two arms was not significantly different. A similar result, although not significant, was obtained in a test involving a choice of PTMinfested-potato and DBM-infested pea plant. More parasitoids (58.2\%) chose the arm with the potato related odour, whereas they spent with $49.5 \%$ the same amount of time as in the arm with the pea related odour.

D. semiclausum was clearly attracted to the cabbage plant. When an undamaged cabbage plant was tested against DBM-infested pea plant, $73.5 \%$ of the females took the cabbage as their first choice, and spent $75.7 \%$ of the time in this arm. The response to cabbage was even stronger when the cabbage plant was infested with DBM with $83.6 \%$ of the first choices made on the cabbage arm and $79 \%$ of the time spent (Fig. 3.6). 

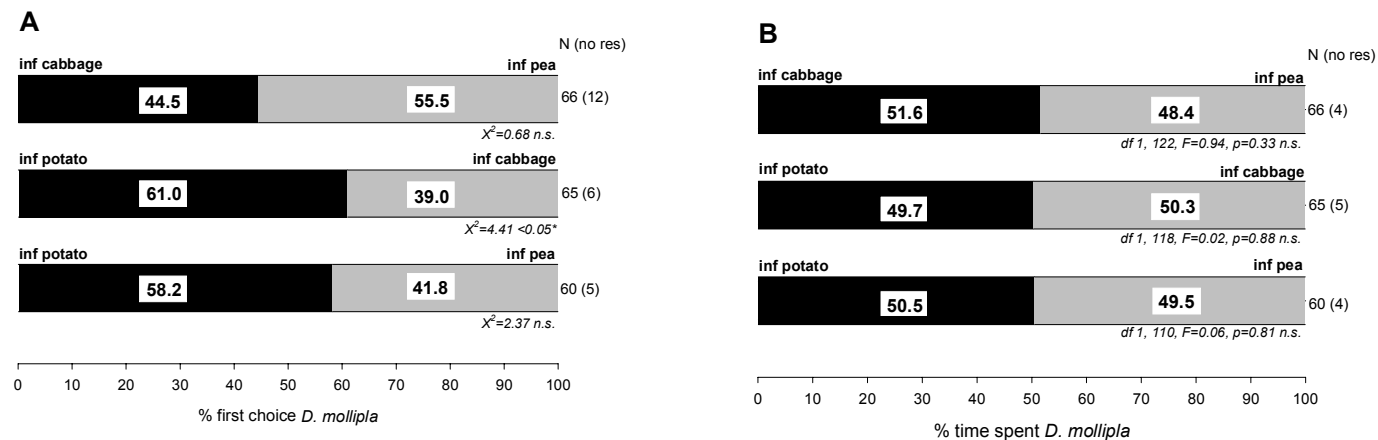

Fig. 3.5: Responses of naive Diadegma mollipla females to infested plants (c-DBMinfested cabbage plant $=$ inf cabbage, -DBM-infested pea plant $=$ inf pea, PTMinfested potatoes $=$ inf potato). A) percentage of the number of first choices made. B) percentage time spent in the arms with respective odours within observation period of 5 min. $\mathrm{N}=$ number of females. Number of parasitoids that did not respond are indicated in brackets. Asterisks indicate statistically significant preferences within tests (Yates corrected $\mathrm{Chi}^{2}$-Test for first choice and Tukey Test for time spent) $\left({ }^{*} \mathrm{p}<0.05,{ }^{* *} \mathrm{p}<0.01\right.$, n.s. $=$ not significant $)$.

A
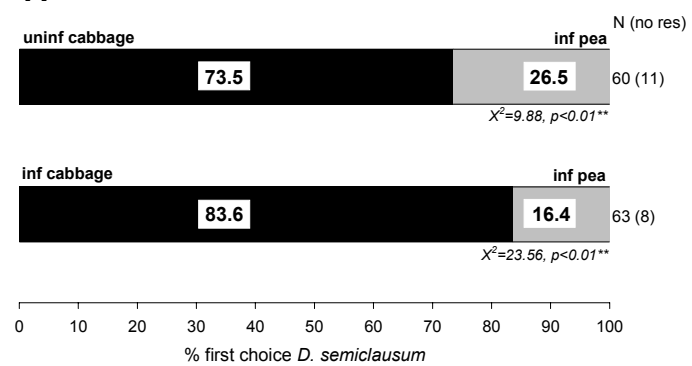

B

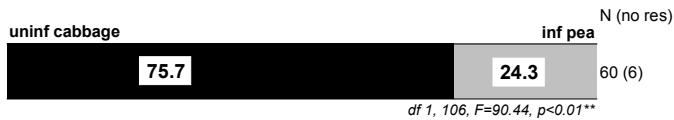

inf cabbage

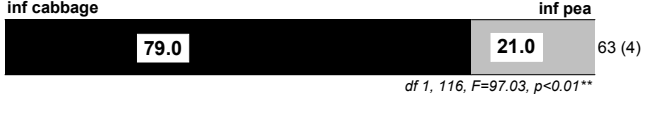

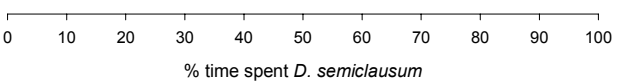

Fig. 3.6: Responses of naive $D$. semiclausum females to infested and uninfested plants (c-DBM-infested cabbage plant $=$ inf cabbage, $\mathrm{p}$-DBM-infested pea plant $=$ inf pea, uninfested cabbage plant $=$ uninf cabbage). A) percentage of the number of first choices made. B) percentage time spent in the arms with respective odours within observation period of $5 \mathrm{~min}$. $\mathrm{N}=$ number of females. Number of parasitoids that did not respond are indicated in brackets. Asterisks indicate statistically significant preferences within tests (Yates corr. Chi ${ }^{2}$-Test for first choice and Tukey Test for time spent) $\left({ }^{*} p<0.05\right.$, $* * p<0.01$, n.s. $=$ not significant $)$. 


\subsection{Discussion}

The host shift of DBM to snowpeas has different effects on the two parasitoids. Diadegma semiclausum relies on volatiles associated with cabbage, DBM's original host, but not with snowpeas. The parasitoid responded to all cabbage-associated odour sources, c-DBM larvae, their faeces and the plant itself (in increasing order). The relative indifference towards DBM-infested snowpea and the avoidance of p-DBM larvae implies lack of attractive volatiles and their precursors from this plant.

Ohara et al. (2003) had already demonstrated that the specialisation of D. semiclausum on DBM is facilitated by crucifer-typical volatiles (perhaps derivates of glucosinolate), which are used by the parasitoid as cues for locating its host. DBM has been known so far as crucifer-specific and the adults use these volatiles to find their host plant (Pivnick et al., 1994). Several other DBM specific parasitoids were also shown to respond to them. Cotesia plutellae Kurdjumov (Hymenoptera: Braconidae) and Trichogramma chilonsis Ishii (Trichogrammatoidea) were attracted to a blend of cabbage volatiles of either intact or/and damaged cabbage plants (Bogahawatte and van Emden, 1996, Potting et al., 1999, Reddy et al., 2002). Examples from other crucifer-herbivore specific parasitoids attracted by isothiocyanates (mustard oils) are Diaeretiella rapae, parasitoid of the aphid Brevicoryne brassicae (Bradburne and Mithen 2000) or Platygaster subuliformis, parasitoid of Dasineura brassicae (Murchie et al., 1997).

The increased attractiveness of the DBM-cabbage complex as compared to the undamaged plant is probably due to the release of additional quantities of isothiocyanates and other volatile components induced through feeding. In many studies, the host-plant complex has been demonstrated to have a stronger effect on host location than the intact plant (e.g. Shiojiri et al., 2000, Takabayashi et al., 1998, Xu et al., 2001). Davis (1987) showed that D. semiclausum preferred an artificially damaged or DBM-infested cabbage leaf over an undamaged leaf. Faeces of c-DBM and c-DBMlarvae were less preferred than infested cabbage. However, they still showed significant attraction relative to clean air. Frass from DBM feeding on cabbage has been shown to contain volatile isothiocyanates, among other components (di- and trisulfides) (Auger et 
al., 1989), which are also present in the cabbage plant itself. Thus, the parasitoid was only attracted by the DBM larvae that were feeding or had fed on cabbage. Host larvae have been shown to attract parasitoids (reviewed in Steidle and van Loon, 2003), but little is mentioned about the influence of the substrate they feed on. In one of the few studies on the influence of larval diet on parasitoids, Havill and Raffa (2000) showed that Glyptapanteles flavicoxis (Marsh), a braconid parasitoid of the gypsy moth Lymantria dispar (L.), was attracted to the larvae when reared on foliage of the host plant, but they were not attracted by larvae reared on an artificial diet. Sex pheromones were also shown to serve as cues (e.g. Reddy et al., 2002), but they do not seem to have an effect on D. semiclausum.

Our observations made during the olfactometer tests indicated that D. mollipla females walked randomly when they had a choice between cabbage-related odours and clean air. The DBM-infested cabbage plant was not particularly attractive, nor were the faeces of c-DBM or the larvae alone. On the other hand, DBM-infested snowpea, as well as PTM-infested potatoes were preferred over clean air, indicating that D. mollipla has a broader host range on a variety of plants. Indeed, between DBM-infested snowpea, PTM-infested potato and DBM-infested cabbage no definite choices for one of the odour sources were made. Our results are also consistent with a recent study by Akol et al. (2003) on the effect of different neem insecticide formulations on the attractiveness of $D$. mollipla. They found that the species did not show any significant preference for a water-sprayed cabbage plant infested with DBM over clean air. Interestingly, the watersprayed infested plant was preferred over uninfested plant. It appears that DBM is not the main host of $D$. mollipla and cabbage not a significant source of attractants for this parasitoid. That may explain why parasitation rates of $D$. mollipla on cabbage in the field are relatively low. Since PTM is a host of D. mollipla, the attractiveness of infested potatoes was not surprising. On the other hand, the reasons for the relative attractiveness of infested snowpeas is unexpected. The attractiveness of snowpeas was also confirmed by laboratory experiments where D. mollipla showed significantly higher parasitation rates on DBM on snowpeas as compared to DBM on cabbage (Löhr and Rossbach, 2004). 
Unlike D. semiclausum, it is unlikely that D. mollipla uses crucifer-typical odours to locate its host, as suggested by Akol et al. (2003). It is assumed that generalist natural enemies use general chemical cues that are present in all hosts or their respective food plants (Vet and Dicke, 1992, Godfray, 1994). Accordingly, D. mollipla is either attracted to a non-specific blend of volatiles from a variety of host-infested plants, or it uses other shared cues present in several hosts or host-plant complexes. Steidle et al. (2003) for example found that the generalist parasitoid Lariophagus distinguendus Förster (Pteromalidae) was attracted to chemicals released by mites associated with the host insects, unrelated to the host plant the hosts were feeding on.

In summary, since D. semiclausum depends on crucifer-specific volatiles to find its host, host shift to a different plant family leads to a loss of host location cues, and this seriously affects its searching efficiency. On the other hand, D. mollipla is less dependent on plant volatiles for its host location. Therefore host shift does not necessarily mean a loss of cues, although the nature of cues that mediate in its host location process remain unknown. 


\section{CHAPTER 4}

\section{Does a specialist parasitoid adapt to its host on a new host plant?}

\subsection{Abstract}

The specialist parasitoid Diadegma semiclausum is guided by crucifer-associated cues in order to locate its host, the diamondback moth, Plutella xylostella (L.) (DBM). It had been introduced to Kenya for biological control of DBM on cabbage. The shift of a DBM strain to snowpea (Pisum sativum L.) raised the question whether D. semiclausum could be conditioned to locate and parasitise its host on a new host plant. Diadegma semiclausum was reared on the snowpea-strain of DBM for 3 subsequent paraitoid generations and responsive behaviour, parasitation rates and fitness were investigated. Bioassay tests were conducted in a Y-tube olfactometer with DBM-infested snowpea tested either against clean air or DBM-infested cabbage. Response to infested snowpea increased in the pea-generations of D. semiclausum as compared to the parasitoid reared on the cabbage strain of DBM. Rearing of the parasitoid in host larvae on peas significantly increased the number of larvae parasitised on this host plant in the second generation but there was no further increase in generation 2 and 3. Larval mortality was similar for all parasitoid/DBM combinations on both host plants, but significantly higher mortality occurred in parasitoid pupae from peas. Development time of the parasitoid was slightly prolonged on the pea strain of DBM. The number of females produced by parasitoids reared on the pea strain of DBM was significantly reduced as compared to D. semiclausum reared on the cabbage strain on both host strains. Results show that $D$. semiclausum has the potential to locate its host on a new host plant. Within 3 generations, responsive behaviour towards snowpea could be increased. However, fitness trade-offs, especially an extreme shift in sex ratio to males reduced reproductive success.

Keywords: Diadegma semiclausum, Plutella xylostella, host shift, adaptation, Pisum sativum 


\subsection{Introduction}

Plants mediate the interaction of herbivores and natural enemies and therefore influence the effectiveness of parasitoids (Cortesero et al., 2000). Plant-derived volatiles are often used by parasitoids as cues to find a suitable host (Vet and Dicke, 1992). Searching efficiency as well as parasitation rates of parasitoids that are guided by plant semiochemicals vary with the attractiveness of volatile blends of the particular plant (Benrey et al., 1997, Billqvist and Ekbom, 2001, Bogahawatte and van Emden, 1996, Geervliet et al., 1996, Liu and Jiang, 2003). The use of semiochemicals as reliable host finding cues has developed in a long coevolutionary process and is to a large extent genetically fixed. However, the recognition of host finding cues underlies a certain plasticity in order to cope with a changing environment and ensure survival (Turlings et al, 1993, Monge and Cortesero, 1996). Olfactory learning is one way to enable parasitoids to modify their behavioural responsiveness to host and plant- derived odours (Vet and Groenewold, 1990, Turlings et al, 1993). Pre-adult learning of host-plant cues and preference for the host plant parasitoids were reared on has been demonstrated (Bjorksten and Hoffmann, 1995, Bogahawatte and van Emden, 1996, Barron, 2001, Gandolfi et al., 2003). However, a specialist parasitoid reacts to specific cues and this may be genetically fixed (Steidle et al., 2003). Learning of infochemical cues of the host plant the parasitoid was reared on might be of little adaptive value for a specialist and therefore, it would not be expected to adapt to a new host plant.

In this study we investigated whether a specialist parasitoid can be conditioned to maintain its searching efficiency by learning new host plant cues. This is of practical importance for biocontrol because parasitoids can lose specific cues provided by the plant when their herbivore host shifts to a different food plant. The parasitoid $D$. semiclausum Hellen (Hymenoptera: Ichneumonidae) and its host, the diamondback moth, Plutella xylostella L. (Lepidoptera: Plutellidae) were used. The diamondback moth is a very destructive oligophagous pest on crucifers (Talekar and Shelton, 1993). In 2000, a diamondback moth population was detected on sugar snap- and snowpeas (Pisum sativum L.) in Naivasha, in the Rift Valley of Kenya (Löhr, 2001). Pea is a plant completely outside the normal host range of the diamondback moth, usually restricted 
to the family of Brassicaceae. Diadegma semiclausum is a specialist parasitoid of DBM (Abbas, 1988) and has been succesfully used for controlling diamondback moth on crucifers in various countries and has been introduced to Kenya and other east African countries. It is highly attracted to cabbage, even in the absence of the host, which implies the use of crucifer-derived volatiles for host location (Ohara et al., 2003). Can the response of $D$. semiclausum to plant-derived semiochemicals and the parasitation efficiency be altered when it is reared on the diamondback moth on snowpea? And are there any trade-offs in fitness-related traits (survival, development time and adult size)? Host location, parasitation and fitness of D. semiclausum reared on the pea strain of diamondback moth was studied in order to evaluate its ability to cope with the host plant shift.

\subsection{Material and Methods}

\subsubsection{Host larvae}

The cabbage strain of diamondback moth (DBM) originated from cabbage fields at Wundanyi, Taita Taveta District, Kenya [Altitude: 1650m, 03²6’11S, 038²0’37E], and was reared in the laboratory $\left(\mathrm{T}=23 \pm 2^{\circ} \mathrm{C}\right)$ on potted cabbage plants (Brassica oleracea L. var. capitata (Copenhagen Market)). Monthly collections of diamondback moth larvae and pupae from Wundanyi were constantly added to the culture in order to maintain its fitness. Larvae of the pea strain were collected from a sugar snap pea field (Pisum sativum, var. Oregon sugar pod) near Naivasha in Nakuru District, Kenya [Altitude: $1500 \mathrm{~m}, 00^{\circ} 44^{\prime} 98 \mathrm{~S}, 036^{\circ} 26^{\prime} 27 \mathrm{E}$ ]. Since 2000, the colony has been maintained on potted snowpeas (Pisum sativum, var. Oregon sugar pod). For detailed methodology of rearing see Löhr and Gathu (2002). Diamondback moth larvae reared on cabbage are referred to as c-DBM and those reared on peas as $\mathrm{p}-\mathrm{DBM}$ throughout this text. 


\subsubsection{Parasitoid}

A colony of D. semiclausum was started in October 2001 with pupae imported from the Asian Vegetable Research and Development Center (AVRDC) in Taiwan. Parasitoids were reared on second and third instar DBM larvae on cabbage leaves in perspex cages serving as parasitation chambers $(20 \times 20 \times 25 \mathrm{~cm})$. Parasitized larvae were transfered to plastic boxes covered with nylon mesh for ventilation and fed with cabbage leaves until pupation. Pupae were then removed and kept in separate containers. Emerging parasitoids were released into parasitiation chambers. Honey was provided as a food source for adults. Age of females used for the experiments varied between 2 and 6 days. Throughout this paper D. semiclausum reared on c-DBM are referred to as c-Ds. For experiments parasitoids were reared for three continuous generations on p-DBM on pea leaves, following the same procedure as for rearing on cabbage. They are referred to as $1 \mathrm{p}-D s, 2 \mathrm{p}-D s, 3 \mathrm{p}-D s$.

\subsubsection{Host plants}

For rearing of DBM and all experiments the same plant varieties of cabbage, Brassica oleracea L. var. capitata (Copenhagen Market) and snowpea, Pisum sativum, var. Oregon sugar pod were used. In a plastic-covered greenhouse plants of both species were reared in pots $(2000 \mathrm{ml})$ in a mixture of garden compost, red soil and sand $(2: 1: 1)$ and were ready for use after 6 weeks.

\subsubsection{Parasitation}

Parasitation rates of $D$. semiclausum (c-Ds) reared on diamondback moth larvae on cabbage and three consecutive generations reared on pea $(1 \mathrm{p}-D s, 2 \mathrm{p}-D s, 3 \mathrm{p}-D s)$ were tested under laboratory conditions $\left(\mathrm{T}=23 \pm 2^{\circ} \mathrm{C}\right)$. Single, mated, two to three days old female D. semiclausum were used in the tests. A single cabbage leaf was infested with 40 second instar c-DBM larvae (4 days old). Leaf and larvae were placed in a small plastic container $(15 \times 15 \times 15 \mathrm{~cm})$ and larvae were allowed to settle for two hours. A 
female parasitoid was then released into the container and allowed to parasitise for $24 \mathrm{~h}$. In the pea treatment, a pea leaf of approximately the same size as the cabbage leaf was infested with 40 p-DBM larvae and treated as described above. Fully expanded leaves from four to six week old plants of both plant species were used. After removing the parasitoid the larvae were fed on their respective food plants until reaching pupation. The number of parasitoid pupae in each container was recorded. DBM larvae that died were dissected in order to search for the parasitoid egg or larvae to obtain the total number of parasitized DBM larvae. For each treatment and parasitoid generation the experiment was replicated 20 times.

\subsubsection{Parasitoid fitness}

Parasitoid fitness in the three subsequent generations on pea was measured in terms of duration of development, mortality, sex ratio and adult size of parasitoids. A potted pea or cabbage plant was infested with 100 second instar diamondback moth larvae. A perpex cage $(25 \times 20 \times 20 \mathrm{~cm})$ with a hole in the base was placed on top of the pot. The pot was covered with aluminium foil in order to prevent dropped larvae from disappearing. Three mated parasitoid females, aged 3-5 days, were released into the cage and left for 24 hours. After exposure, the larvae were collected and introduced individually into vials where they were fed on a leaf disc/leaflet of their respective food plant until pupation and kept until emergence. The vials were checked daily and larval and pupal development duration were recorded. Spinning of the cocoon was considered as an indicator of pupation. Mortality was recorded for parasitized and unparasitized larvae. The cause of larval death of parasitized DBM was determined by dissecting the larvae and searching for the parasitoid egg/larvae. Failure of emergence was recorded as pupal death. For each parasitoid generation the experiment was replicated four times. The sex of each emerging parasitoid was recorded and females were kept in $70 \%$ ethanol for determination of adult size. Length of the left hind tibia and the left forewing of 20 females in each pea generation were measured with a micrometer scale using a microscope (Leica MZ8). 


\subsubsection{Behaviour assays}

The olfactory attractiveness of diamondback moth-infested cabbage and snowpea to two different generations of $\mathrm{p}-D$. semiclausum females $(1 \mathrm{p}-D s$ and $3 \mathrm{p}-D s)$ was studied in a Y-tube olfactometer. Individual odour sources were placed in a glass jar with an airtight lid connected to an olfactometer arm by a Teflon tube $(0.5 \mathrm{~cm}$ inner diameter $)$. Air from an inlet pump was passed through an activated charcoal filter for purification, then through a flow meter into two separate odour source jars. A second flow meter was connected between the stem of the olfactometer and a second pump, which exhausted air out of the system. The airflow in the olfactometer was set at $130 \mathrm{ml} / \mathrm{min}$ (inlet) and $250 \mathrm{ml} / \mathrm{min}$ (outlet) respectively.

The following odour source choices were tested. 1) An entire potted snowpea plant (4 weeks old) with 20 feeding larvae was placed in a glass jar of $2000 \mathrm{ml}$ capacity. The larvae were allowed to feed for 20 hours before the experiment. On the other branch of the olfactometer, a glass jar of the same size remained empty. 2) Entire potted plants (4 weeks old) of cabbage and snowpea were infested with 20 larvae. They were allowed to feed for 20 hours before use. The plants were placed in the jars on either side of the olfactometer. The choice tests were conducted with "normal" c-D. semiclausum and the first and third generation of the parasitoid reared on p-DBM.

Two to six day old, naive female parasitoids were introduced individually in the stem of the Y-olfactometer. They were allowed to stay for five minutes. Parasitoids that crossed a line $0.5 \mathrm{~cm}$ beyond the intersection and remained in one arm for at least 20 seconds were recorded as having made a first choice. Total time spent in either one or both arms was recorded during the observation period. Females that remained in the stem or spent less than 20 seconds in one of the arms were recorded as showing no response. Parasitoids that switched between the arms without initially spending more than 20 seconds in one of the arms were recorded as not having made a definite choice but total time spent was still measured. To avoid bias, the odour sources were switched between the left and the right arm of the Y-tube after every ten parasitoids tested. The time spent 
was recorded with the Observer 3.0 for Windows from Noldus IT.

\subsubsection{Statistical analysis}

In order to assess the effect of host plant and parasitoid generation on development and mortality of parasitoids a two-way-ANOVA, with host strain and parasitoid generation as factors was used. Parasitation rates and adult size of the subsequent generations of the parasitoid on pea were compared with the Student Newman Keuls Test. The 0.05 level was set for all comparisons made. The Chi-square adaptation test was used in order to determine deviations of the sex ratio from an expected 1:1 ratio. For comparison of differences in sex ratios between parasitoid/DBM combinations the Chisquare $2 \times 2$ was applied.

The results of the choice between odour sources were analysed with the Yates-corrected Chi-Square test. For differences in time spent in the two indiviual arms the Tukey test was used after an arcsine transformation of data (GLM proc SAS Institute, 1990). Parasitoids that made no choice were excluded from the analyses. Parasitoids that made no definite first choice but spent time in both olfactometer arms were also included in the GLM analyses. For differences between preferences of parasitoid generations a multiple Chi-Square Test was conducted (Freq proc SAS Institute, 1990). 


\subsection{Results}

\subsubsection{Parasitation}

Single females reared on c-DBM parasitised significantly more larvae on cabbage than on pea (df $1, \mathrm{~F}=14.2, \mathrm{p}<0.01$ ) (Table 4.1). Rearing of the parasitoid in host larvae on peas significantly increased the number of larvae parasitised on this host plant in the first generation, but there was no further increase in generation 2 and 3 (df $3, F=15.3$, $\mathrm{p}<0.01$ ) (Table 4.1). When parasitoids reared on pea hosts were offered host larvae reared on cabbage, their parasitation was similar to those reared on cabbage and this did not change with the number of generations on the alternative host plant (pea) (df 3 , $\mathrm{F}=1.33, \mathrm{p}=0.27)$ (Table 4.1).

Table 4.1: Percent parasitism according to wasp generation and host strain (c- $D s: D$. semiclausum emerged from c-DBM, $1 \mathrm{p}-D s, 2 \mathrm{p}-D s, 3 \mathrm{p}-D s: 1^{\text {st, }} 2^{\text {nd }}$ and $3^{\text {rd }}$ generation emerged from p-DBM). Mean of 20 replicates. Data was analyzed by SNK-Test. Different letters indicate significant differences among means of one host strain $(p<0.05)$. P-values show differences between host strains.

\begin{tabular}{lccl}
\hline parasitoid & $\begin{array}{c}\text { Mean c-DBM [\%] } \\
\text { parasitized }\end{array}$ & $\begin{array}{c}\text { Mean p-DBM [\%] } \\
\text { parasitized }\end{array}$ & p \\
\hline $\mathrm{c}-D s$ & $62.8 \pm 18.3 \mathrm{a}$ & $40.0 \pm 21.0 \mathrm{a}$ & $<0.001 * *$ \\
$1 \mathrm{p}-D s$ & $73.6 \pm 15.8 \mathrm{a}$ & $74.5 \pm 16.8 \mathrm{~b}$ & $=0.85$ n.s. \\
$2 \mathrm{p}-D s$ & $65.3 \pm 17.5 \mathrm{a}$ & $70.1 \pm 15.8 \mathrm{~b}$ & $=0.34$ n.s. \\
$3 \mathrm{p}-D s$ & $68.4 \pm 21.5 \mathrm{a}$ & $68.8 \pm 21.1 \mathrm{~b}$ & $=0.94$ n.s.
\end{tabular}




\subsubsection{Parasitoid mortality}

In Table 4.2 mortality of parasitized and unparasitized DBM larvae is shown. Two-way ANOVA showed a significant difference between pupal mortality on the two host plants (df $1, F=14.1, p<0.01$ ). Pupal mortality was consistently higher on peas than on cabbage, irrespective of the parasitoid generation. Larval mortality of parasitized larvae was not significantly different between parasitoids or host strain ( $d f 1, F=0.38, p=0.38$ ). In exposed yet not parasitised larvae and pupae no significant differences were found for all plant host/parasitoid combinations ( $\mathrm{df} 1, \mathrm{~F}=0.47, \mathrm{p}=0.49$ and $\mathrm{df} 1, \mathrm{~F}=0.29$, $p=0.59)$. Higher mortality was entirely due to higher pupal deaths in the larvae raised on peas. The non-exposed hosts in the control group (both plant species) were similar to the exposed group on cabbage. Similar parasitation rates for each parasitoid/host combination (61.9 to $77.1 \%$ ) indicate that the larvae were equally disturbed by parasitoids and differences in mortality were not caused by differential stress-related deaths.

Table 4.2: Mortality of parasitized and unparasitized DBM in relation to host strain (cDBM, p-DBM) and wasp generation (c-Ds: D. semiclausum emerged from c-DBM, 1p$D s, 2 \mathrm{p}-D s: 1^{\text {st, }}$ and $2^{\text {nd }}$ generation emerged from $\mathrm{p}$-DBM). Mean of at least four replicates. Data was analyzed by Two-Way ANOVA. Different letters indicate significant differences among means $(\mathrm{p}<0.05)$.

\begin{tabular}{|c|c|c|c|c|c|}
\hline \multirow[t]{2}{*}{ parasitoid } & \multirow[t]{2}{*}{ DBM } & \multicolumn{2}{|c|}{ Mortality parasit. DBM } & \multicolumn{2}{|c|}{ Mortality unparasit. DBM } \\
\hline & & larval & pupal & larval & pupal \\
\hline c-Ds & $\mathrm{c}$ & $2.8 \pm 3.1 \mathrm{a}$ & $9.0 \pm 7.7 \quad \mathrm{a}$ & $4.0 \pm 2.1 \mathrm{a}$ & $1.8 \pm 1.5 \mathrm{a}$ \\
\hline $1 \mathrm{p}-D s$ & $\mathrm{c}$ & $14.0 \pm 4.0 \mathrm{a}$ & $11.5 \pm 3.7 \quad \mathrm{a}$ & $9.3 \pm 9.2 \mathrm{a}$ & $0.5 \pm 0.6$ \\
\hline $2 \mathrm{p}-D s$ & $\mathrm{c}$ & $5.3 \pm 3.7 \mathrm{a}$ & $9.0 \pm 1.8 \mathrm{a}$ & $2.8 \pm 0.5 \mathrm{a}$ & $2.0 \pm 1.8$ \\
\hline $\mathrm{c}-D s$ & $\mathrm{p}$ & $6.8 \pm 2.5 \mathrm{a}$ & $18.7 \pm 11.0 \mathrm{~b}$ & $9.8 \pm 4.7 \mathrm{a}$ & $3.0 \pm 3.3$ \\
\hline $1 \mathrm{p}-D s$ & $\mathrm{p}$ & $11.3 \pm 7.2 \mathrm{a}$ & $24.3 \pm 5.6 \quad b$ & $3.3 \pm 2.5 \mathrm{a}$ & $2.0 \pm 1.1$ \\
\hline $2 \mathrm{p}-D s$ & $\mathrm{p}$ & $4.4 \pm 3.8 \mathrm{a}$ & $23.8 \pm 12.5 b$ & $3.2 \pm 4.5 \mathrm{a}$ & $4.2 \pm 4.1$ \\
\hline- & $\mathrm{c}$ & - & & $2.0 \pm 1.0 \mathrm{a}$ & $2.0 \pm 1.0$ \\
\hline- & $\mathrm{p}$ & - & & $9.5 \pm 0.7 \mathrm{a}$ & $5.0 \pm 4.2$ \\
\hline
\end{tabular}




\subsubsection{Parasitoid fitness}

In Table 4.3 development time of parasitoids and of healthy DBM is presented. DBM larvae were exactly 5 days old when exposed to the parasitoids. In order to compare larval development time of parasitized with unparasitized DBM development duration was counted from day 5 (exposure day). Spinning of the cocoon was considered as the beginning of the pupation period. Development time of parasitized larvae depended on the host strain. On p-DBM larval and pupal development were significantly longer than on $\mathrm{c}-\mathrm{DBM}$ ( $\mathrm{df} 1, \mathrm{~F}=9.84, \mathrm{p}<0.01$ and $\mathrm{df} 1, \mathrm{~F}=13.31, \mathrm{p}<0.01$ ). There was no significant difference between parasitoid generations on the same host. Unparasitized c-DBM completed their larval stage significantly faster than unparasitized p-DBM (df 1, $\mathrm{F}=17.42, \mathrm{p}<0.01)$. Pupal development was similar for all parasitoid/host strain combinations df $1, \mathrm{~F}=1.4, \mathrm{p}=0.26$ ). Undisturbed DBM larvae (control) showed similar larval and pupal development times as to the parasitoids exposed, but unparasitized larvae. Compared to unparasitized DBM larval duration of the parasitized larvae was extended significantly in both host strains ( $\mathrm{df} 1, \mathrm{~F}=57.62, \mathrm{p}<0.01)$.

The sex ratio of progeny was male-biased for all parasitoid/host combinations (Table 4.3). However, parasitoids reared on cabbage hosts produced significantly more female offspring than parasitoids raised on pea irrespective of the host plant the offered larvae were reared on. Female ratio on pea was consistently at $10 \%$ or below irrespective of host plant of the offered larvae and the number of generations the parasitoid had passed through pea host larvae.

Diamondback moth host strain significantly affected the size of emerging adult parasitoids (Table 4.4). On average, the left forewing of females reared on cabbage larvae was $0.5 \mathrm{~mm}$ and the left hind leg was $0.15 \mathrm{~mm}$ longer than that of those reared on the pea strain. 
Table 4.3: Development duration and sex ratio of parasitized and unparasitized DBM in relation to host strain (c-DBM, p-DBM) and wasp generation (c-Ds: D. semiclausum emerged from c-DBM, $1 \mathrm{p}-D s, 2 \mathrm{p}-D s: 1^{\text {st. }}$ and $2^{\text {nd }}$ gen. emerged from $\mathrm{p}-\mathrm{DBM}$ ). Mean of at least 4 replicates. Data for development was analyzed by Two-Way ANOVA. Different letters indicate significant differences among means $(\mathrm{p}<0.05)$. Differences in sex ratio between parasitoid strains was analyzed by Chi-Square Test and same symbols indicate similar ratios $\left(\dagger,{ }^{\circ}\right)$.

\begin{tabular}{|c|c|c|c|c|c|c|}
\hline \multirow[t]{2}{*}{ parasitoid } & \multirow[t]{2}{*}{ host } & \multicolumn{2}{|c|}{ Development time [days] } & \multicolumn{2}{|c|}{$\begin{array}{c}\text { Development time [days] } \\
\text { unparasit. DBM }\end{array}$} & \multirow{2}{*}{$\begin{array}{c}\text { Sex } \\
\text { ratio } \\
\\
q\end{array}$} \\
\hline & & larval & pupal & larval & pupal & \\
\hline $\mathrm{c}-D s$ & $\mathrm{c}$ & $4.6 \pm 0.7 \mathrm{a}$ & $10.6 \pm 0.4 \mathrm{a}$ & $4.0 \pm 0.7 \mathrm{a}$ & $5.1 \pm 0.2 \mathrm{a}$ & $0.32 \dagger$ \\
\hline $1 \mathrm{p}-D s$ & $\mathrm{c}$ & $4.8 \pm 0.4 \mathrm{a}$ & $10.8 \pm 0.4 \mathrm{a}$ & $4.0 \pm 0.3 \mathrm{a}$ & $4.6 \pm 0.4 \mathrm{a}$ & $0.10^{\circ}$ \\
\hline $2 \mathrm{p}-D s$ & $\mathrm{c}$ & $4.4 \pm 0.2 \mathrm{a}$ & $10.1 \pm 0.4 \mathrm{a}$ & $3.7 \pm 0.1 \mathrm{a}$ & $4.8 \pm 0.7 \mathrm{a}$ & $0.08^{\circ}$ \\
\hline $\mathrm{c}-D s$ & $\mathrm{p}$ & $5.4 \pm 0.4 \mathrm{~b}$ & $10.0 \pm 0.2 \mathrm{~b}$ & $4.5 \pm 0.3 b$ & $4.9 \pm 0.4 \mathrm{a}$ & $0.46 \dagger$ \\
\hline $1 \mathrm{p}-D s$ & $\mathrm{p}$ & $5.7 \pm 0.3 b$ & $9.9 \pm 0.5 b$ & $4.5 \pm 0.1 b$ & $5.3 \pm 0.5 \mathrm{a}$ & $0.07^{\circ}$ \\
\hline $2 \mathrm{p}-D s$ & $\mathrm{p}$ & $5.2 \pm 0.3 \mathrm{~b}$ & $9.5 \pm 0.4 b$ & $4.4 \pm 0.3 b$ & $4.8 \pm 0.5 \mathrm{a}$ & $0.07^{\circ}$ \\
\hline - & $\mathrm{c}$ & - & - & $4.3 \pm 0.1 \mathrm{a}$ & $5.4 \pm 0.1 \mathrm{a}$ & - \\
\hline - & $\mathrm{p}$ & - & - & $4.5 \pm 0.2 b$ & $5.2 \pm 0.6 \mathrm{a}$ & - \\
\hline
\end{tabular}

Table 4.4: Body size of parasitoid females in relation to wasp generation and host strain (c-Ds: D. semiclausum emerged from c-DBM, $1 \mathrm{p}-D s, 2 \mathrm{p}-D s, 3 \mathrm{p}-D s: 1^{\text {st, }} 2^{\text {nd }}$ and $3^{\text {rd }}$ generation emerged from p-DBM). Mean of 20 replicates. Data was analyzed by SNKTest. Different letters indicate significant differences among means $(\mathrm{p}<0.05)$.

\begin{tabular}{lcc}
\hline \multicolumn{1}{c}{ parasitoid } & Mean wing length $[\mathbf{m m}]$ & Mean tibia length $[\mathbf{m m}]$ \\
\hline $\mathrm{c}-D s$ & $3.51 \pm 0.17 \mathrm{a}$ & $1.14 \pm 0.06 \mathrm{a}$ \\
$1 \mathrm{p}-D s$ & $3.02 \pm 0.15 \mathrm{~b}$ & $0.99 \pm 0.05 \mathrm{~b}$ \\
$2 \mathrm{p}-D s$ & $3.09 \pm 0.16 \mathrm{~b}$ & $1.03 \pm 0.09 \mathrm{~b}$ \\
$3 \mathrm{p}-D s$ & $3.08 \pm 0.19 \mathrm{~b}$ & $1.03 \pm 0.08 \mathrm{~b}$ \\
\hline
\end{tabular}




\subsubsection{Host location}

"Normal"on cabbage reared $D$. semiclausum females did not differ significantly from those raised for one and three generations on $\mathrm{p}$-DBM larvae $(1 \mathrm{p}-D s, 3 \mathrm{p}-D s)$ in their first choice between pea and clean air ( $\left.\mathrm{df} 2, \mathrm{X}^{2}=2.67, \mathrm{p}=0.26\right)$ (Fig. 4.1A). Females of $1 \mathrm{p}-D s$ and $3 p-D s$ were equally distributed between the two olfactometer arms. In the third generation of the parasitoid on p-DBM a slight preference for infested pea was observed but it remained below significance level. Time spent in the different arms of the olfactometer was similar between normal parasitoids and after one generation on peas when infested pea was tested against clean air (Fig. 4.1B). Although females of the third generation, had spent a significantly longer time span on the side of the pea odour than with clean air, no significant difference was found for time spent between the three tested generations (df 2, $\left.\mathrm{X}^{2}=1.72, \mathrm{p}=0.42\right)$ (Fig. 4.1B).

When the parasitoids were given the alternative odours of infested cabbage and infested pea, first choice shifted significantly from infested cabbage in c- $D s$ to infested pea in $3 \mathrm{p}-D s\left(\mathrm{df} 2, \mathrm{X}^{2}=44.9, \mathrm{p}<0.01\right)$ (Fig. 4.2A). Parasitoids reared on $\mathrm{c}-\mathrm{DBM}$ showed a strong and significant preference for the cabbage odour. After one generation on pea host larvae, females equally went to cabbage odour and pea odour. After 3 generations on pea more females chose infested pea over infested cabbage. Probably because of the lower number of test females, the choice preference remained below significance level in the third generation (Fig. 4.2A). Fewer animals were tested, because it was difficult to produce a sizable amount of females over three generations. All test females spent significantly more time on the cabbage side than pea, irrespective of their upbringing and number of generation on the alternative host larvae (Fig. 4.2B). However, the time spent in the arm with infested pea increased significantly from c- $D s$ to the generations on pea $\left(\mathrm{df} 2, \mathrm{X}^{2}=7.95, \mathrm{p}<0.05\right)$. 
A

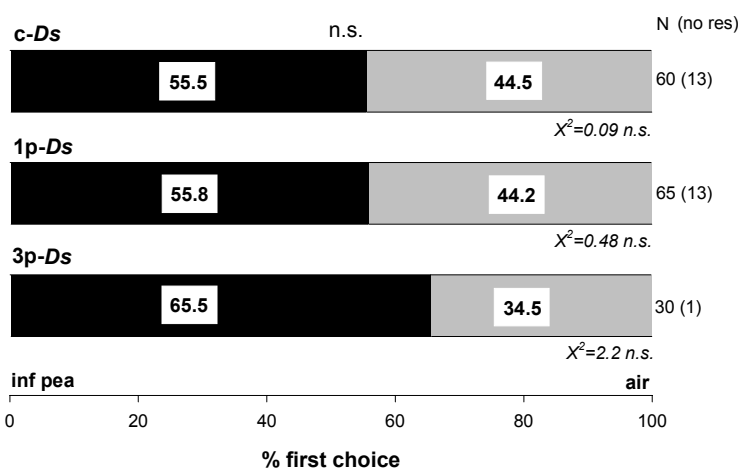

B

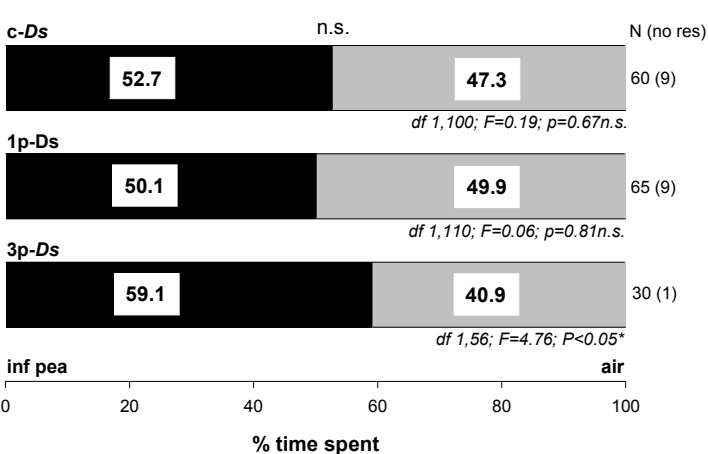

Fig. 4.1: Responses of female parasitoid generations (c-Ds, $1 \mathrm{p}-D s$ and $3 \mathrm{p}-D s$ ) to DBM infested snowpea tested against clean air. A) percentage of the number of first choices made. B) percentage time spent in the arms with respective odour within observation period of $5 \mathrm{~min}$. $\mathrm{N}=$ number of females. Number of parasitoids that did not respond are indicated in brackets. Asterisks indicate statistically significant preferences within tests (below bars) and between test generations (above bars) $(* p<0.05, * * p<0.01$, n.s. $=$ not significant).

A
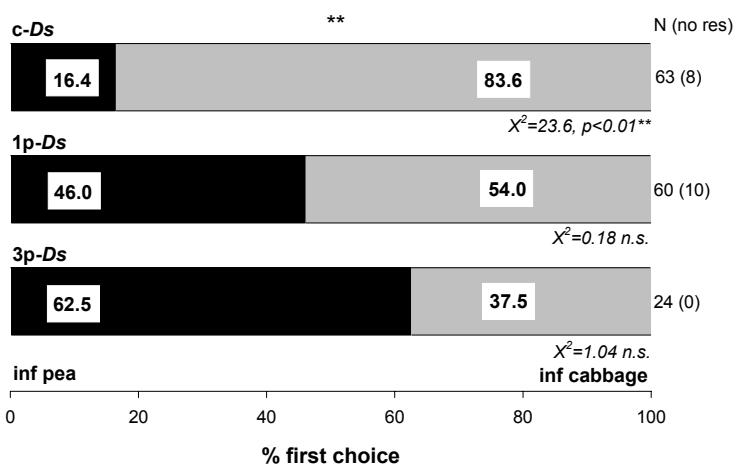

B

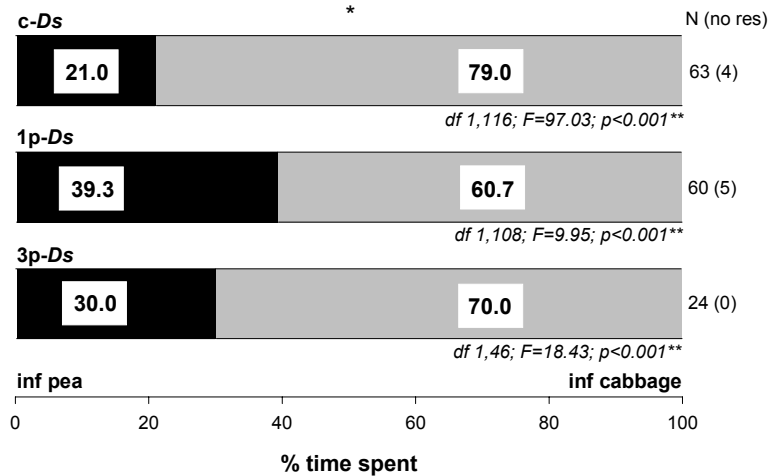

Fig. 4.2: Responses of female parasitoid generations (c-Ds, $1 \mathrm{p}-D s$ and $3 p-D s$ ) to $\mathrm{p}$ DBM infested snowpea tested against c-DBM infested cabbage. A) percentage of the number of first choices made. B) percentage time spent in the arms with respective odour within observation period of $5 \mathrm{~min}$. $\mathrm{N}=$ number of females. Number of parasitoids that did not respond are indicated in brackets. Asterisks indicate statistically significant preferences within tests (below bars) and between test generations (above bars) $(* \mathrm{p}<0.05, * * \mathrm{p}<0.01$, n.s. $=$ not significant $)$. 


\subsection{Discussion}

Diadegma semiclausum is a specialist parasitoid, which normally uses crucifer-typical volatiles as host finding cues (Ohara et al., 2003). We showed that the parasitoid can alter its response to plant volatiles when reared on an unusual host plant. The level of attraction to infested snowpea increased in the pea generations of the parasitoid as compared to the females reared on cabbage. First choice shifted from infested cabbage (for $\mathrm{c}-D s$ ) to infested snowpea (for $3 \mathrm{p}-D s$ ) when the two plant odours were tested against each other. However, regarding the time spent in these odours, preference for the normal host plant remained. Still, females reared on the pea host spent relatively more time in the arm with the snowpea odour than females reared on the cabbage strain. When tested against clean air, the relative increase in attraction to infested snowpea was not as clear, but third generation pea parasitoids still tended to be attracted more to the novel host plant than the females reared on the cabbage host strain. Recognition of chemical cues for host location can be genetically fixed (Vet and Dicke 1992), but parasitoids often learn host- or host plant derived stimuli to help them to find their host (Turlings et al., 1993). The degree of learning of infochemical cues is expected to depend on the dietary specialisation of the parasitoid and its host (Steidle et al., 2003). Some parasitoids prefer odours from the host plant they were reared on. This was demonstrated for Cotesia plutellae (Kurdjumov), which was more attracted to the brassica type on which they had developed (Bogahawatte and van Emden, 1996). Learning of olfactory cues takes place during particular stages of the development of a parasitoid. Some species require an initial stimulus during adult emergence that is provided by their host plant. For example, parasitoid adults of bruchids developing in Leguminosae seeds need to have contact with seeds in order to respond to volatiles of these seeds (Monge and Cortesero, 1996). Similarly, Hérard et al. (1988) found that contact upon emergence with cocoons containing frass of the plant was an important source of stimulation for Microplitis demolitor Wilkinson. The ability of parasitoids to learn olfactory cues during the preimaginal stage has only recently been conclusively proven by Gandolfi et al. (2003). They demonstrated that Hyssopus pallidus (Askew), an ectoparasitoid of the codling moth, a pest on apple fruits, was able to learn even cues of a host- and fruit-independent odour (menthol) during its larval development. 
Parasitoids exposed to menthol odour at larval stages no longer showed negative responses as adults. Learning of chemical cues seemed to play a role for the behavioural response to plant odours of $D$. semiclausum. Exposing the parasitoid to novel plant attributes during its larval development or during emergence could have induced the shift in attraction towards chemical stimuli of snowpea. Even though pupae were removed from their host plants and kept in a plastic container until emergence, frass and tiny plant residues still remained on the the silky surface of the cocoons. Contact upon emergence with this plant material might have triggered the first "impulse" of $D$. semiclausum reared on snowpea to choose the odour of infested pea over infested cabbage. However, parasitoids reared on pea still stayed significantly longer on the side with the cabbage odour. This indicates that innate cues probably predominate in $D$. semiclausum and the response to plant volatiles can only be changed within a limited range. Innate cues might be too strong to be overcome by a few generations on an unusual host plant. Although the change in attractiveness of infested snowpea led to higher parasitation of the pea strain of DBM by parasitoids reared on pea, these "primed" parasitoids parasitized equally well on the cabbage strain of DBM.

Rearing of D. semiclausum on the pea strain of DBM had consequences regarding parasitoid fitness. Development and growth potential of parasitoids are indirectly influenced by the host plant through physiology and size of the herbivore (Godfray, 1994). In our experiments, development and growth of parasitized larvae were clearly determined by the growth potential of the DBM host strain. Wasp progeny emerging from larvae reared on peas were smaller and developed slightly slower than wasps emerging from the cabbage strain. We already demonstrated that development of the parasitoid D. mollipla was prolonged on snowpeas as compared to cabbage and larvae of the cabbage strain of DBM were slightly bigger and pupae heavier than those of the pea strain (see Chapter 2). The origin of the parent wasp (c-Ds or $\mathrm{p}-D s$ ) had no influence on development time and adult size. But interestingly, the origin of the parent female determined the sex ratio of progeny, regardless of the progeny's host strain. Offspring of D. semiclausum reared on pea was extremely male-biased on both DBM strains, whereas the parasitoid reared on cabbage produced significantly more females again on both host strains. Biased sex ratio is usually attributed to factors like host 
quality (Godfray, 1994), differential mortality of male and female offspring during immature development (Charnov et al., 1981) or plant quality, e.g. nutrient content, secondary compounds (Godfray, 1994, Fox et al., 1996, Campan and Benrey, 2004). Host quality as a factor for a direct choice by the female whether to oviposit a fertilized or an unfertilized egg would have resulted in host strain dependent sex ratio. Nor was differential mortality the cause for the bias in sex-ratio. Mortality of $\mathrm{p}-D s$ on the pea strain of DBM was not higher than of $\mathrm{c}-D s$ on the cabbage DBM strain. Unfavourable physiological properties of snowpea seemed to have reduced reproductive fitness of $D$. semiclausum reared on pea. Legumes are rich in allelochemicals, eg. proteinase inhibitors, saponins, non-protein amino acids. Allelochemicals might have affected sperms negatively. Howeer, we found no evidence for this possibility. Another reason could be incomplete or insufficient mating and therefore a low proportion of fertilized eggs.

Differences in mortality of parasitized DBM larvae resulted from a significantly higher proportion of parasitoids that failed to emerge from cocoons of the pea strain, regardless of the host strain of the parent wasp. Larval survival was not affected by the host strain. Parasitoid pupal mortality on the pea strain could have been caused by host quality and size as well as a shorter pupal period that did not allow parasitoids to develop fully. Often secondary plant metabolites (allelochemicals) have detrimental effects on parasitoid development and survival (Barbosa et al., 1986, 1991, Campos et al., 1990, Roth et al., 1997) and could have affected D. semiclausum. Snowpea could also lack nutritional compounds required for a successful development of the parasitoid.

Diadegma semiclausum has the potential to locate its host on a new host plant. Within three generations, responsive behaviour towards snowpea could be increased substantially. However, the effects were short lived and unlikely to alter the female's behaviour in an adaptive way in just a few generations of rearing on a new host plant. Still, D. semiclausum could adapt over time to the new host-plant complex, maybe as a step in a speciation process (see Cronin and Abrahamson, 2001). However, fitness trade-offs would have to be overcome. Especially the reduction in reproductive success due to the extreme shift in sex ratio to males hampers an adaptation of $D$. semiclausum 
to the new host plant of the diamondback moth. 


\section{CHAPTER 5}

\section{Interspecific competition between Diadegma semiclausum Hellen and Diadegma mollipla (Holmgren) on Plutella xylostella (L.) feeding on an unusual host plant.}

\subsection{Abstract}

Interspecific competition of an introduced and a local parasitoid of the diamondback moth (DBM), Plutella xylostella on an unusual host plant was investigated. The specialist Diadegma semiclausum was introduced to Kenya for biocontrol of the diamondback moth on cabbage. When the normally crucifer-typical diamondback moth included snowpeas in its diet in Kenya laboratory experiments with the most important local parasitoid Diadegma mollipla resulted in higher parasitation levels of its host on snowpeas as compared to cabbage. Diadegma semiclausum was more efficient on cabbage and clearly out-competed its congenus on the original host plant. We hypothesized a shift in competitiveness between the two parasitoids on the new host plant. In the laboratory simultaneous and delayed competition was tested on snowpeas and additonally the influence of host plants under different host and parasitoid densities was investigated in the greenhouse. Performance of the two parasitoid species in the laboratory differed largely from cage experiments in the greenhouse. Confined to small containers with a high density of hosts, D. mollipla was more competitive and parasitized equally as well or even better than D. semiclausum. When given more space D. mollipla parasitized very few larvae. Parasitation levels of D. semiclausum were significantly higher than those of D. mollipla, regardless of host plant, host and parasitoid densities, but progeny production of D. mollipla on snowpea was still slightly higher than on cabbage. The cages supposingly represented field conditions more closely and might give a better picture of the parasitoids' behaviour in their natural environment.

Keywords: competition, host shift, D. semiclausum, D. mollipla, P. xylostella, peas 


\subsection{Introduction}

Interspecific competition among insect parasitoids can influence the size and structure as well as the stability of insect communities. Introduced parasitoids are often stronger competitors and can even displace other parasitoids (reviewed in Reitz and Trumble, 2002). Likely candidates for introduction as biocontrol agents have been the subject of investigation on interspecific competition. In many studies differential larval competition and the ability of host discrimination were found to effect direct competitivness between two parasitoid species (McBrien and Mackauer, 1990, Pijls et al., 1995, Bokonon-Ganta et al., 1996, Infante et al., 2001, Agboka et al., 2002, Wang and Messing, 2003). Individual searching efficiency can also change when two parasitoid species compete for the same host (Chua et al., 1990). Only few studies about the effect of host plants on interspecific competition between parasitoids exist (Iwao et al., 2001). Host plants indirectly influence the impact of natural enemies on their herbivore host (Cortesero et al., 2000). Levels of attack rates can differ on different host plants because plants often mediate host location efficiency and therefore influence the effectiveness of parasitoids (Benrey at al., 1997, Billquist and Ekbom, 2001, Liu and Jiang, 2003). Due to differential host location abilities competitiveness of two parasitoids on different host plants might be different. Therefore the influence of a new host plant of a herbivore host on an introduced and a local competing parasitoid was investigated.

In 2002 the specialist parasitoid Diadegma semiclausum Hellen (Hymenoptera: Ichneumonidae) was introduced to Kenya in order to suppress diamondback moth, Plutella xylostella L. (Lepidoptera: Plutellidae) populations in cabbage production areas in the Kenyan highlands. The most important local parasitoid was Diadegma mollipla (Holmgren) (Hymenopera: Ichneumonidae), a generalist parasitoid that together with other local parasitoids never exceeded parasitation levels above 15\% (Odour et al., 1996). Both species parasitize the same larval stages, have similar temperature requirements, but differ in their host specifity. With the potato tuber moth, Phthorimaea operculella (Zeller) (Lepidoptera: Gelechiidae) one other host is known for the indigenous D. mollipla, but additional hosts are suspected. Diadegma semiclausum is 
specific for the diamondback moth (DBM) (Abbas, 1988) and has been the most widely used and successful parasitoid for biocontrol of DBM in a number of countries (Talekar and Shelton, 1993). In the laboratory Sithole (2004) demonstrated a clear advantage in competition of D. semiclausum over D. mollipla on DBM on cabbage. Observations in the field confirmed that the exotic species out-competed and even displaced most of the generalist indigenous species on DBM on cabbage including D. mollipla (unpublished data). When the normally crucifer-specific DBM extended its host range to snowpeas in 2000 in a horticulture production area in Naivasha in the Rift Valley of Kenya, efficiency in parasitation of D. mollipla on DBM on the new host plant was investigated and the species proved to parasitize better on DBM on snowpeas than on cabbage (Löhr and Rossbach, 2004). Host location studies revealed that D. mollipla could find its host equally as well on cabbage and on peas, whereas $D$. semiclausum relied on crucifertypical volatiles as host-finding cues and prefered cabbage over DBM infested snowpea (see Chapter 3). Therefore we hypothized that on the new host plant D. mollipla might have a competitive advantage over its introduced congenus and might be able to find a niche on peas after its displacement on cabbage. Progeny production under simultaneous and delayed interspecific competition was determined in the laboratory. In the greenhouse the influence of host plants on competition and the effect of different host and parasitoid densities was investigated.

\subsection{Material and methods}

\subsubsection{Host plants and larvae}

The cabbage strain of diamondback moth (DBM) originated from cabbage fields at Wundanyi, Taita Taveta District, Kenya [Altitude: 1650m, 0326’11S, 038²0’37E], and was reared in the insectory $\left(\mathrm{T}=23 \pm 2^{\circ} \mathrm{C}\right)$ on potted cabbage plants (Brassica oleracea L. var. capitata (Copenhagen Market)). The pea strain of diamondback moth was collected from a sugar snap pea field (Pisum sativum, var. Oregon sugar pod) near 
Naivasha in Nakuru District, Kenya [Altitude: 1500m, 0044’98S, 036²6’27E]. Since 2000, the colony has been maintained on potted snowpeas (Pisum sativum, var. Oregon sugar pod) in the laboratory. For detailed description of rearing of both DBM strains see Löhr and Gathu (2002).

\subsubsection{Parasitoids}

A culture of D. mollipla was started from collected DBM larvae in Naro Moru, Nyeri District, Kenya. Parasitoids were multiplied for three generations in the laboratory before they were used for experiments. A colony of D. semiclausum was started in October 2001 with pupae imported from the Asian Vegetable Research and Development Center (AVRDC) in Taiwan. The culture had been maintained in the laboratory for three years when experiments were conducted. Both species were reared separately on second and third instar DBM larvae on cabbage leaves in perspex cages serving as parasitation chambers $(20 \times 20 \times 25 \mathrm{~cm})$. Parasitized larvae were transfered to plastic boxes covered with nylon mesh for ventilation and fed with cabbage leaves until pupation. Pupae were then removed and kept in separate containers. Emerging parasitoids were released into parasitation chambers. Honey was provided as a food source for adults. Age of females used for the experiments varied between 2 and 5 days and both species had no oviposition experience before the experiments.

\subsubsection{Simultaneous intra- and interspecific competition}

Three plastic oviposition chambers $(15 \times 15 \times 15 \mathrm{~cm})$ were prepared and to each were added 50 DBM larvae on a pea leaf. They were given 2 hours to settle on the leaf. To two of the chambers two mated females, both belonging to either D. mollipla or $D$. semiclausum, were released and to the third chamber one female from each of the two species was added. After 24 hours the parasitoids were removed and the larvae were reared to adults. The number of parasitoid pupae and progeny for each species emerging at the end of the experiment was recorded. Dead larvae were dissected in order to check 
for parasitoid eggs/larvae. The experiment was conducted at $23 \pm 2{ }^{\circ} \mathrm{C}$ and replicated fifteen times.

\subsubsection{Delayed simultaneous interspecific competition}

In the same oviposition chambers used in the experiment above fifty $2^{\text {nd }}$ instar larvae on a pea leaf were exposed to a single female of a D. semiclausum female. At two different times (after $4 \mathrm{~h}$ and $8 \mathrm{~h}$ ) D. mollipla was introduced without removing the first female. Total oviposition time was $24 \mathrm{~h}$. The experiment was repeated in reverse order of parasitoids and was replicated ten times for each time sequence and species order.

\subsubsection{Delayed interspecific competition}

Fifty $2^{\text {nd }}$ instar larvae on a pea leaf were exposed for 24 hours to oviposition by a single D. semiclausum female in an oviposition chamber. Larvae were transferred to a fresh pea leaf and then exposed to subsequent 24 hours parasitization by D. mollipla at different times. That was immediately, 24 hours and 48 hours after exposure to $D$. semiclausum. Each time sequence was replicated 10 times. The number of parasitoid pupae and progeny for each species emerging at the end of the experiment was recorded. Dead larvae were dissected in order to check for parasitoid eggs/larvae. In the second part of the experiment the larvae were first exposed to D. mollipla and then to $D$. semiclausum.

\subsubsection{Effect of host plants on interspecific competition}

This experiment was conducted in cages in a plastic sheet-covered greenhouse. The cages had plastic frames $(110 \times 90 \times 75 \mathrm{~cm})$ covered with a fine mesh on all sides and the top. On the ground of the cages a wooden board $(80 \mathrm{x} 100 \mathrm{~cm})$ with four openings $(\varnothing 15 \mathrm{~cm})$ was placed. The board was covered with a layer of soil $(1 \mathrm{~cm})$ and was 
elevated from the ground in order to insert potted plants into the openings. Distance between plants was $60 \mathrm{~cm}$ and $40 \mathrm{~cm}$. Diagonal distance was $75 \mathrm{~cm}$. This set-up was chosen in order to simulate field conditions as closely as possible.

DBM on one host plant and two host plants were offered in three different sets of experiments. First four potted cabbage plants were introduced into the cage. A day before parasitoid exposure each plant was infested with 20 second instar DBM larvae reared on cabbage. Then two females of each species were released into the cage for 8 hours (exposure time $8.30 \mathrm{am}$ to $4.30 \mathrm{pm}$ ). Larvae were collected immediately after the end of exposure time and reared until emergence. Number of parasitoid pupae and species and sex of emerged adults was recorded. The experiment was repeated with DBM reared on peas on four pea plants and finally two infested pea plants and two infested cabbage plants were offered at the same time. Every host plant exposure was replicated six times ( 3 cages at a time).

\subsubsection{Effect of larval density on competition}

This experiment was conducted with two host plants (two pea and two cabbage plants) in above described cages. In the first part the cabbage plants were infested with 20 larvae and the pea plants with 10 larvae. Afterwards the density of larvae on the two host plant species was reversed. Otherwise the procedure was exactely as described above.

\subsubsection{Effect of parasitoid density on competition}

Two snowpea and two cabbage plants infested with 20 DBM larvae each were introduced into the cage (procedure as described above). The experiment was conducted with three different $D$. mollipla densities. The number of $D$. mollipla females was two, four and in the last set-up six. The number of $D$. semiclausum females was kept constant with two. 


\subsubsection{Statistical analyses}

All data was analyzed using the SAS loglinear modeling GENMOD procedure (SAS Institute 1999-2000). The model is particularly suitable for count data under the assumption of a Poisson distribution. In cases the criteria for Goodness of Fit deviated too far from 0 (value $>1.5$ ) the Poisson regression was replaced by a negative binomial regression with the same SAS procedure. For the presentation of parasitation levels at different host densities the data was arcsin transformed before analysis.

\subsection{Results}

\subsubsection{Simultaneous competition}

Fig. 5.1A shows the of number parasitized larvae, total progeny and females from three different species combinations when larvae were exposed to two competing females at the same time. Intraspecific competition between two females of D. mollipla and interspecific competition between the two species had a similar number of parasitized larvae and produced a higher number of progeny than two competing $D$. semiclausum (df $\left.2, X^{2}=7.14, p<0.05\right)$. The number of female progeny was very variable within all parasitoid combinations and were therefore not significantly different from each other (df $2, \mathrm{X}^{2}=4.13, \mathrm{p}=0.12$ ).

Fig. 5.1B shows the contribution of each species to production of progeny in interspecific combination of D. mollipla and D. semiclausum. The mean number of emerged adults of $D$. mollipla was double the amount of progeny produced by $D$. semiclausum. However, the difference was not significant, because of the high standard deviation ( $\mathrm{df} 1, \mathrm{X}^{2}=3.04, \mathrm{p}=0.08$ ). The number of females produced by D. mollipla was significantly higher than by $D$. semiclausum $\left(\mathrm{df} 1, \mathrm{X}^{2}=11.1, \mathrm{p}<0.01\right)$. 

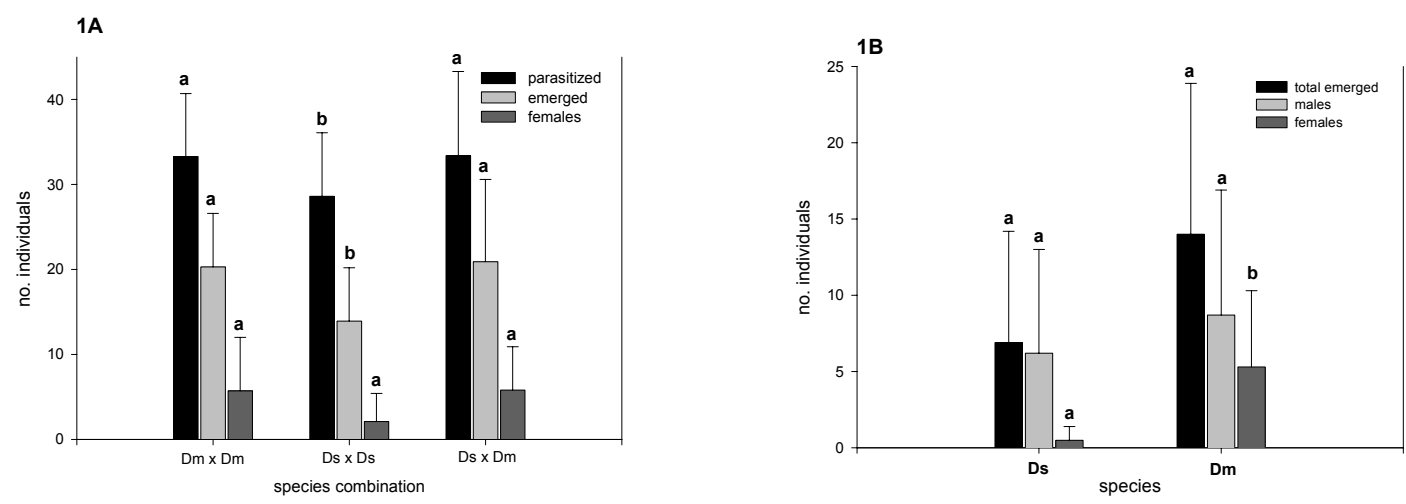

Fig. 5.1: Simultaneous intra- and interspecific competition of Diadegma mollipla (Dm) and Diadegma semiclausum (Ds) on Plutella xylostella on snowpea. A) No. of parasitized larvae, total progeny production and emerged females B) Progeny production of both species in interspecific competition.

\subsubsection{Delayed simultaneous competition}

Parasitation and progeny production of D. mollipla and D. semiclausum within $24 \mathrm{~h}$ with a head start of $4 \mathrm{~h}$ and $8 \mathrm{~h}$ for one or the other species are presented in Table 5.1. Combined number of parasitized larvae was similar for every exposure sequence. When D. semiclausum was given a head start, the total number of progeny was significantly lower as compared to D. mollipla starting to attack earlier ( $\mathrm{df} 3, \mathrm{X}^{2}=6.74, \mathrm{p}<0.01$ ). Individual ovipositing females performed very variably. When D. mollipla attacked earlier the number of progeny of each species was similar after $24 \mathrm{~h}$ of oviposition. When this species was introduced later, it produced significantly less progeny than $D$. semiclausum ( $\mathrm{df} 1, \mathrm{X}^{2}=5.35, \mathrm{p}<0.05$ after $4 \mathrm{~h}$ and $\mathrm{df} 1, \mathrm{X}^{2}=9.33, \mathrm{p}<0.01$ after $8 \mathrm{~h}$ ). The number of female progeny was very low for both species. 
Table 5.1: Delayed simultaneous interspecific competition of Diadegma mollipla (Dm) and Diadegma semiclausum (Ds) on Plutella xylostella on snowpea. The $2^{\text {nd }}$ parasitoid was introduced after $4 \mathrm{~h}$ and $8 \mathrm{~h}$ respectively. Total parasitation duration was $24 \mathrm{~h}$. Different letters indicate significant difference between exposure sequence and introduction time of $2^{\text {nd }}$ parasitoid and asterix between the species $(p<0.05)$.

\begin{tabular}{|c|c|c|c|c|c|c|}
\hline \multirow{2}{*}{$\begin{array}{c}\text { Exposure } \\
\text { sequence } \\
{[\mathrm{h}]}\end{array}$} & \multirow{2}{*}{$\begin{array}{c}\text { No. parasit. } \\
\text { DBM }\end{array}$} & \multirow{2}{*}{$\begin{array}{l}\text { No. emerged } \\
\text { parasitoids }\end{array}$} & \multicolumn{2}{|c|}{ D. mollipla } & \multicolumn{2}{|c|}{ D. semiclausum } \\
\hline & & & total & females & total & females \\
\hline $\begin{array}{l}\text { Dm - Ds } \\
{[4]}\end{array}$ & $32.4 \pm 8.2 \mathrm{a}$ & $24.8 \pm 9.7 \mathrm{a}$ & $10.5 \pm 10.1 \mathrm{a}$ & $4.2 \pm 7.4 \mathrm{a}$ & $14.3 \pm 12.1 \mathrm{a}$ & $1.6 \pm 3.7 \mathrm{a}$ \\
\hline $\begin{array}{l}\text { Dm - Ds } \\
{[8]}\end{array}$ & $26.6 \pm 6.7 \mathrm{a}$ & $21.4 \pm 5.1 \mathrm{a}$ & $7.5 \pm 9.8$ & $1.2 \pm 3.1 \mathrm{a}$ & $13.9 \pm 9.1 \mathrm{a}$ & $2.7 \pm 2.1 \mathrm{a}$ \\
\hline $\begin{array}{l}\text { Ds - Dm } \\
{[4]}\end{array}$ & $28.9 \pm 4.7 \mathrm{a}$ & $10.8 \pm 7.9 \mathrm{~b}$ & $2.8 \pm 2.7$ & $0.6 \pm 0.8 \mathrm{a}$ & $8.0 \pm 6.9 \quad \mathrm{a} *$ & $1.0 \pm 2.1 \mathrm{a}$ \\
\hline $\begin{array}{l}\text { Ds - Dm } \\
{[8]}\end{array}$ & $29.0 \pm 13.4 \mathrm{a}$ & $18.9 \pm 14.9 \mathrm{ab}$ & $1.0 \pm 1.9$ & $0.1 \pm 0.3 \mathrm{a} *$ & $17.9 \pm 14.3 \mathrm{a} *$ & $\begin{array}{c}5.5 \pm 6.6 \mathrm{a} \\
*\end{array}$ \\
\hline
\end{tabular}

\subsubsection{Delayed competition}

The number of parasitized larvae was similar for all exposure sequences (Table 5.2). The time interval of exposure affected total and individual progeny production. Significantly less adults emerged at time intervals of $48 \mathrm{~h}$ and $72 \mathrm{~h}$, regardless which parasitoid species attacked larvae first $\left(\mathrm{df} 5, \mathrm{X}^{2}=41.5, \mathrm{p}<0.01\right)$. The number of progeny of each species was very variable and the time interval had no significant effect on the total number of progeny of both species, except for D. mollipla at $72 \mathrm{~h}$ (df $5, \mathrm{X}^{2}=36.64$, $\mathrm{p}<0.01)$. However, at time intervals of $48 \mathrm{~h}$ and $72 \mathrm{~h}$, the species which attacked first produced more progeny, but that was only significant at $72 \mathrm{~h}$ ( $\mathrm{df} 1, \mathrm{X}^{2}=4.39, \mathrm{p}<0.05$ for Dm-Ds and df $1, X^{2}=36.28, p<0.01$ for Ds-Dm). The number of females produced was very low and variable between individual ovipositing females and exposure sequence nor time interval had an influence on female progeny. 
Table 5.2: Delayed interspecific competition of Diadegma mollipla (Dm) and Diadegma semiclausum (Ds) on Plutella xylostella on snowpea. Time intervals of exposure were $24 \mathrm{~h}, 48 \mathrm{~h}$ and $72 \mathrm{~h}$ between parasitoids. Different letters indicate significant difference between exposure sequence and time interval of exposure and asterix indicate significant differences between the species $(\mathrm{p}<0.05)$.

\begin{tabular}{|c|c|c|c|c|c|c|}
\hline \multirow{2}{*}{$\begin{array}{c}\text { Expo- } \\
\text { sequence } \\
{[\mathrm{h}]}\end{array}$} & \multirow{2}{*}{$\begin{array}{c}\text { No. parasit. } \\
\text { DBM }\end{array}$} & \multirow{2}{*}{$\begin{array}{l}\text { No. emerged } \\
\text { parasitoids }\end{array}$} & \multicolumn{2}{|c|}{ D. mollipla } & \multicolumn{2}{|c|}{ D. semiclausum } \\
\hline & & & total & females & total & females \\
\hline $\begin{array}{l}\text { Dm - Ds } \\
{[24]}\end{array}$ & $37.3 \pm 5.8 \mathrm{a}$ & $25.1 \pm 3.7 \mathrm{a}$ & $12.1 \pm 9.6 \mathrm{a}$ & $2.0 \pm 4.0 \mathrm{a}$ & $13.0 \pm 9.7 \quad \mathrm{a}$ & $3.3 \pm 5.8 \mathrm{a}$ \\
\hline $\begin{array}{l}\text { Dm - Ds } \\
{[48]}\end{array}$ & $36.7 \pm 6.1 \mathrm{a}$ & $19.4 \pm 4.7 \mathrm{~b}$ & $12.6 \pm 7.4 \mathrm{a}$ & $3.3 \pm 3.8 \mathrm{a}$ & $6.8 \pm 5.8 \quad \mathrm{a}$ & $0.9 \pm 2.5 \mathrm{a}$ \\
\hline $\begin{array}{l}\text { Dm - Ds } \\
{[72]}\end{array}$ & $33.8 \pm 9.6 \mathrm{a}$ & $17.1 \pm 7.3 \mathrm{~b}$ & $11.4 \pm 6.9 \mathrm{a} *$ & $1.1 \pm 2.3 \mathrm{a}$ & $5.7 \pm 3.6 \quad \mathrm{a} *$ & $1.4 \pm 2.0 \mathrm{a}$ \\
\hline $\begin{array}{l}\text { Ds - Dm } \\
{[24]}\end{array}$ & $33.8 \pm 7.4 \mathrm{a}$ & $26.4 \pm 8.2 \mathrm{a}$ & $12.2 \pm 8.2 \mathrm{a}$ & $6.5 \pm 7.1 \mathrm{a} *$ & $14.2 \pm 13.4 \mathrm{a}$ & $1.0 \pm 1.7 \mathrm{a} *$ \\
\hline $\begin{array}{l}\text { Ds - Dm } \\
{[48]}\end{array}$ & $28.4 \pm 14.5 b$ & $17.4 \pm 9.5 \mathrm{~b}$ & $5.9 \pm 6.6 \mathrm{a}$ & $2.1 \pm 3.0 \mathrm{a}$ & $11.5 \pm 10.4 \mathrm{a}$ & $0.9 \pm 2.2 \mathrm{a}$ \\
\hline $\begin{array}{l}\text { Ds - Dm } \\
\text { [72] }\end{array}$ & $30.3 \pm 5.6 \mathrm{a}$ & $16.6 \pm 7.9 b$ & $0.3 \pm 0.5 b^{*}$ & $0.0 \pm 0.0 \mathrm{a} *$ & $16.3 \pm 7.9 \mathrm{a} *$ & $2.7 \pm 3.5 \mathrm{a} *$ \\
\hline
\end{tabular}

\subsubsection{Effect of host plants on interspecific competition}

In all cage experiments conducted in the greenhouse D. mollipla was far less competitive than in the laboratory tests. When DBM infested pea plants were offered alone or mixed with infested cabbage plants D. mollipla parasitized less than 1 larvae per plant as compared to an average offspring of 7 of D. semiclausum (Table 5.3). When cabbage was offered alone all progeny was produced by $D$. semiclausum. Contribution of parasitoid species to the total number of progeny in the exposure of both host plants together is not presented separately because it did not differ from the the figures shown in Table 5.3. The number of parasitized larvae was equally distributed between plants. On both plant species seven adults emerged from an average of 8 parasitized larvae. Progeny production of D. mollipla on cabbage was 0.3 and on 
pea 0.6 per plant. Diadegma semiclausum produced significantly more individuals $(6.6$ on pea and 6.9 on cabbage).

Table 5.3: Effect of host plants on interspecific competition of Diadegma mollipla (Dm) and Diadegma semiclausum (Ds) on Plutella xylostella. Different letters indicate significant differences between host plant combination and asterix indicate significant differences between the species $(\mathrm{p}<0.05)$.

\begin{tabular}{|c|c|c|c|c|c|c|c|}
\hline \multirow{3}{*}{$\begin{array}{l}\text { Host plant } \\
\text { cabbage }\end{array}$} & \multirow{3}{*}{$\begin{array}{c}\begin{array}{c}\text { No. parasit. } \\
\text { DBM }\end{array} \\
9.6 \pm 3.0 \text { a }\end{array}$} & \multirow{3}{*}{$\begin{array}{c}\begin{array}{c}\text { No. emerged } \\
\text { parasitoids }\end{array} \\
8.1 \pm 2.6 \text { a }\end{array}$} & \multicolumn{3}{|c|}{ D. mollipla } & \multicolumn{2}{|c|}{ D. semiclausum } \\
\hline & & & \multicolumn{2}{|c|}{ total } & \multirow{2}{*}{$\begin{array}{c}\text { females } \\
0\end{array}$} & \multirow{2}{*}{$\begin{array}{c}\text { total } \\
8.1 \pm 2.6 \mathrm{a}^{*}\end{array}$} & \multirow{2}{*}{$\begin{array}{c}\text { females } \\
0.9 \pm 1.3 \mathrm{a}\end{array}$} \\
\hline & & & 0 & $a^{*}$ & & & \\
\hline pea & $10.5 \pm 3.4 \mathrm{a}$ & $8.0 \pm 3.6 \mathrm{a}$ & $0.8 \pm 1.3$ & $b *$ & 0 & $7.3 \pm 3.4 \mathrm{a} *$ & $0.5 \pm 0.8$ \\
\hline cabbage/pea & $8.3 \pm 4.4 \mathrm{a}$ & $7.2 \pm 3.9 \mathrm{a}$ & $0.5 \pm 0.7$ & $b *$ & 0 & $6.8 \pm 3.7 \mathrm{a} *$ & $0.3 \pm 0.6$ \\
\hline
\end{tabular}

\subsubsection{Effect of host density on interspecific competition}

Level of parasitation and progeny production were not influenced by a different number of larvae on host plants. On the same plant percentage parasitized larvae, emerged adults and progeny contribution of parasitoid species were similar for both host densities (10 and 20 larvae/plant). In Table 5.4 results are therefore summarized in relation to the host plant. The contribution of D. mollipla to the total progeny production was much lower on both plants, but it was significantly higher on pea than on cabbage. 
Table 5.4: Effect of host density and host plants on interspecific competition of Diadegma mollipla (Dm) and Diadegma semiclausum (Ds) on Plutella xylostella. Host densities: 10 and 20 larvae on both plants (c-10 and c-20 on cabbage, p-10 and p-20 on pea). Different letters indicate significant differences between host densitites plant combination and asterix indicate significant differences between the species (arcsin transformed, $\mathrm{p}<0.05)$.

\begin{tabular}{|c|c|c|c|c|c|c|}
\hline \multirow{2}{*}{$\begin{array}{l}\text { host } \\
\text { plant }\end{array}$} & \multirow{2}{*}{$\begin{array}{c}\text { \%. parasit. } \\
\text { DBM }\end{array}$} & \multirow{2}{*}{$\begin{array}{l}\% \text { emerged } \\
\text { parasitoids }\end{array}$} & \multicolumn{2}{|c|}{ \% D. mollipla } & \multicolumn{2}{|c|}{ \% D. semiclausum } \\
\hline & & & total & females & total & females \\
\hline cabbage & $61.7 \mathrm{a}$ & $48.1 \mathrm{a}$ & $0.5 \mathrm{a}$ & 0 & 99.5 a & $39.5 \mathrm{a}$ \\
\hline pea & $47.4 \mathrm{a}$ & $38.4 \mathrm{a}$ & $10.8 \mathrm{~b}$ & 0 & $89.2 \mathrm{~b}$ & $35.1 \mathrm{a}$ \\
\hline
\end{tabular}

\subsubsection{Effect of parasitoid density on interspecific competition}

An increase in the number of females of D. mollipla did not result in an increase of progeny of this species, but in a decrease of progeny of D. semiclausum on the pea plants (Fig. 5.2). The number of D. mollipla offspring was consistently low and remained below 1 larva/per plant on both host plants. On cabbage plants total number of parasitized larvae, production of progeny and contribution of parasitoid species to total progeny was similar for all parasitoid densities (Fig. 5.2A). The number of progeny produced by $D$. semiclausum was significantly higher and did not change with an increasing number of ovipositing $D$. mollipla females. On pea plants higher number of D. mollipla females resulted in a significant reduction of number of parasitized larvae and production of progeny, due to a reduction of D. semiclausum progeny from 6.6 (equal number of ovipositing females) to 1.2 (six D. mollipla females) (Fig. 5.2B). 


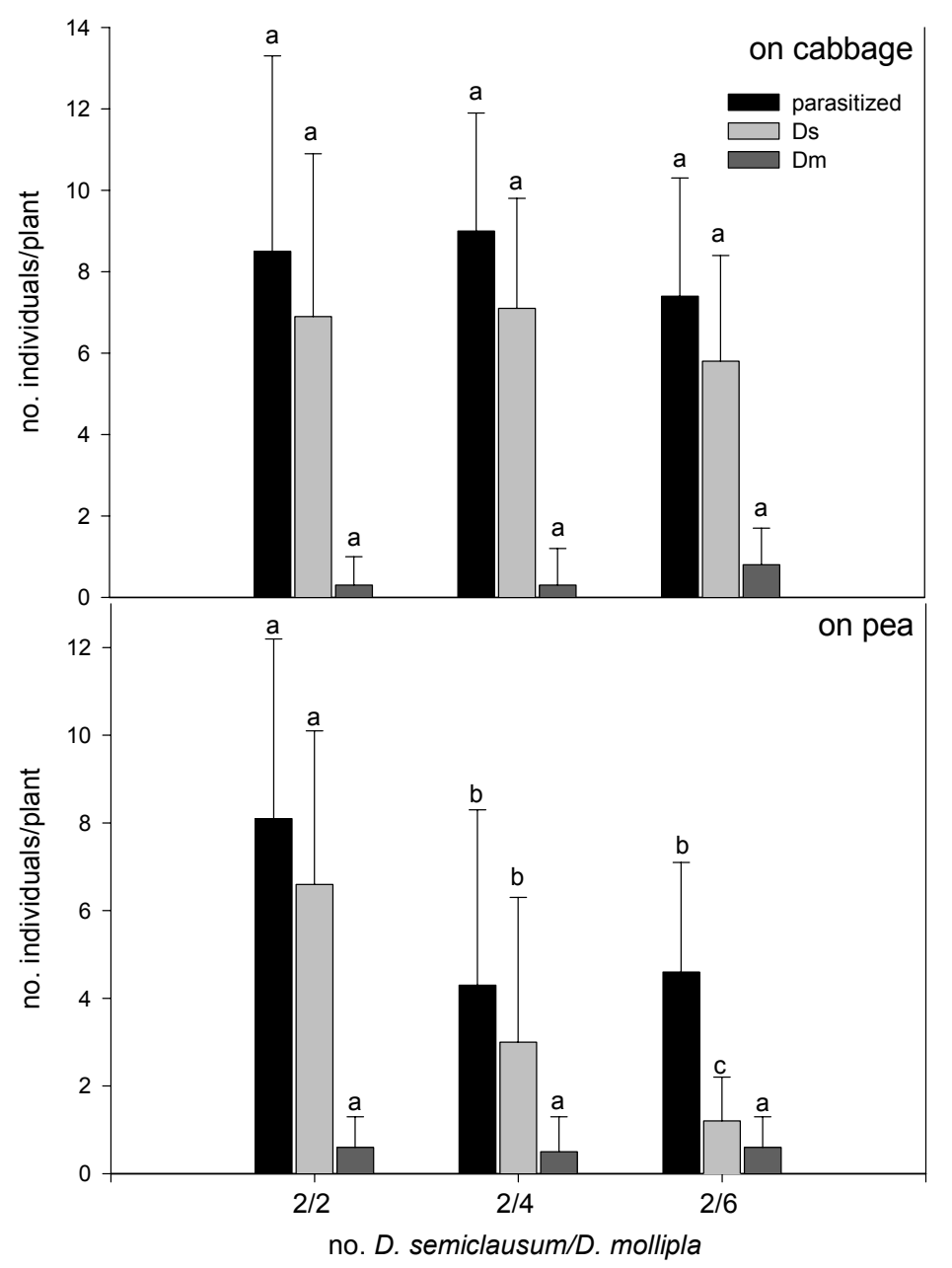

Fig. 5.2: Effect of Diadegma mollipla density on DBM offered on cabbage and pea as host plants on interspecific competition with $D$. semiclausum. Number of females: $2 / 2$ (two D. mollipla females, $2 / 4$ (four D. mollipla), 2/6 (six D. mollipla) and two D. semiclausum. 


\subsection{Discussion}

In simultaneous intra- and interspecific competition on snowpea D. mollipla was superior to D. semiclausum. It produced significantly more total progeny than $D$. semiclausum and slightly more females although that was only significant under interspecific parasitation. In an identical experimental set-up on cabbage as host plant D. semiclausum clearly out-competed D. mollipla, producing $73 \%$ of total progeny (Sithole 2004). Both species are able to discriminate between parasitized and unparasitized larvae (Lloyd, 1940, Ullyet, 1943, 1947, Venkatraman, 1964, Yang et al., 1994). We assume that almost all progeny emerged from singely parasitized larvae and competition took place between adults depending on which species parasitized more hosts within the given time. Mortality of parasitized hosts was mainly attributed to failure of emergence. Only very few hosts died in their larval stage and they contained a single parasitoid embryo. Higher mortality of pupae from snowpeas as compared to cabbage had been found for both species (Rossbach et al., submitted, and unpublished data). Higher parasitation levels of Diadegma mollipla on DBM on snowpea as compared to DBM on cabbage has been demonstrated in an earlier study (Löhr and Rossbach, 2004), whereas for D. semiclausum the reverse was true (Rossbach et al., submitted). The specialist $D$. semiclausum uses crucifer-typical volatiles to locate its host (Ohara et al., 2003) and has therefore a higher host location ability on cabbage than on pea. Host location cues of D. mollipla are unknown, but the species seem to use a blend of volatiles existing in a variety of plants (see Chapter 3). But D. mollipla lost its advantage in performance it showed during simultaneous competition on snowpea when it started to attack a few hours later than its competitor. When this species attacked first it produced equally as many progeny after $24 \mathrm{~h}$ as $D$. semiclausum but it failed to parasitize as many larvae when $D$. semiclausum started to attack first. Diadegma semiclausum produced similar numbers of progeny, independent of which species was given a head start. Freshly parasitized larvae by D. semiclausum seemed to have disturbed D. mollipla in parasitizing. Some parasitoids deposit an external signal after oviposition. The parasitoid Aphidius smithi for example marks its hosts externally with pheromones. Mackauer (1990) suggested that these marking pheromones on already parasitized hosts could be detected by antennation and facilitate discrimination. The 
ectoparasitoid Eupelmus vuilleti (Crw) probably leaves a chemical on the surface of the seeds containing parasitized hosts (Gauthier et al., 1999). A different mechanism of chemical interference is used by Cephalonomia stephanoderis and Prorops nasuta (Hymenoptera: Bethylidae). Adults emit a characteristic smell, presumably to keep away other individals (Infante et al., 2001). Chemical interference might play a role in the interaction of $D$. mollipla and $D$. semiclausum, although we have no evidence of the existance of chemical markers. Results from delayed competition with increasing time interval between exposures of parasitoids support this theory. On cabbage, whichever of the two species had first access to the host, irrespective of exposure interval, predominated with 85 to $97 \%$ of the emerging progeny (Sithole, 2004). On snowpea the first attacker was only the clear winner when the time interval was greater than $48 \mathrm{~h}$. Hosts which had been parasitized by either species longer ago seemed to have negatively affected the ability of the other to locate unparasitized larvae for parasitation. Time interval of attacks also played a role in performance of parasitoids Aphidius ervi and A. smithi. Rejection of parasitized pea aphids by the other species increased with time interval of attacks (McBrian and Mackauer, 1990). A change in host physiology of older parasitoid embryos was suggested, but the nature of this change was not further discussed. In our study the number of parasitized DBM larvae was constant for all exposure sequences and time intervals (except for Ds-Dm [48h]), but total progeny was significantly reduced at time intervals of $48 \mathrm{~h}$ and $72 \mathrm{~h}$, irrespective which species parasitized first. After these time intervals host larvae were already in their third to fourth instar. Most likely higher pupal parasitoid mortality was caused by a shorter development duration that remained for the second attacker rather than multiparasitism of hosts.

The ability of a species to produce proportionally more females than its competitor is crucial for its performance (Reitz and Trumble, 2002). However, we did not place great emphasis on female progeny in order to measure performance, because of the differential time both species were kept in culture and the concomitant decline in females in laboratory cultures. Furthermore the number of females was extremely variable for both species in all experiments and did not allow an interpretation of 
results.

Results from the laboratory implied an advantage for D. mollipla over D. semiclausum on snowpea as compared to cabbage and we assumed that this species would find its niche on snowpea whereas D. semiclausum remained predominant on cabbage. But under more natural conditions in the cages $D$. mollipla performed much poorer than confined to small containers. As expected, all progeny on cabbage, when offered alone, came from $D$. semiclausum. But on pea alone progeny production of $D$. mollipla did not increase to higher numbers than one individual per plant, whereas D. semiclausum produced a constant number of offspring on both plants, whether offered alone or together.

At different host densities on mixed host plants $D$. mollipla still produced significantly less progeny than its competitor, but it parasitized better on their hosts on snowpea, regardless of the number of larvae available. An increasing number of ovipositing $D$. mollipla females did not lead to a higher production of total offspring. But on snowpeas D. semiclausum parasitized significantly less larvae when its competitor was present in higher numbers. The presence of "too many" females let D. semiclausum shun away from the unusual host plant.

On the whole D. mollipla performed slighly better on snowpea than on cabbage, but remained less competitive than $D$. semiclausum. However, it still might be able to parasitize on snowpeas even in the presence of the overall predominant $D$. semiclausum, especially when it occurs in higher numbers. It will have to be tested in the field,whether D. mollipla can establish on DBM in snowpeas after its displacement on cabbage or it will simply withdraw to its other hosts. 


\section{CHAPTER 6}

\section{Parasitoid diversity of Plutella xylostella L. feeding on an unusual host plant}

\subsection{Abstract}

A population of the diamondback moth Plutella xylostella (L.) (Lepidoptera: Plutellidae) (DBM), known to be an oligophagous pest on crucifers, was recently found to infest sugar snap- and snowpeas in the Rift Valley in Kenya, causing heavy damage. The effect local parasitoids on DBM in snowpeas was studied in the field, including the newly introduced parasitoid Diadegma semiclausum. Snowpeas provided an enemy-free space for DBM. Local parasitoids attacked DBM only sporadically and in very low numbers (0-3.5 individuals/20 plants) on DBM in the pea fields as compared to DBM on kales (up to 48 individuals/20 plants) . In both crops the most abundant parasitoid was Oomyzus sokolowskii.

After the release of D. semiclausum the number of DBM in kales reduced drastically to less than 2 individuals/20 plants, but the parasitoid had little effect on DBM on snowpeas. Percentage parasitism on snowpeas went from 2.3 to $4 \%$, whereas on kales it increased from 25.6 to $75.7 \%$.

Keywords: Plutella xylostella, parasitoids, host shift, Pisum sativum, enemy-free space 


\subsection{Introduction}

Utilization of novel host plants by herbivores can provide a refuge from natural enemies (Jeffries and Lawton, 1984) and therefore host plant shifts and increases in diet breadth have been suggested to be facilitated by the interaction with natural enemies (Bernays and Graham, 1988). Reduction of natural enemy attack through aquisition of a new host plant has been demonstrated by various authors (Feder et al., 1995, Gratton and Welter, 1999, Gross et al., 2004, Mulatu et al., 2004). Enemy-free space on a new host plant can be the consequence of plant-associated physiological and behavioural adaptations of parasitoids. Chemical and morphological plant attributes can influence the foraging success of parasitoids as well as the role of plants as host finding cues (Cortesero et al., 2000). The shift to a novel host plant can result in a loss of plant-related cues and to a reduced searching efficiency, especially of specialist parasitoids (Brown et al., 1995). On the other hand, generalist parasitoids which do not rely as strongly on plantmediated host location may still be able to find their host on a different plant (see Chapter 3).

In 1999, in the Rift Valley in Kenya, a population of the diamondback moth (DBM) Plutella xylostella L. (Lepidoptera: Plutellidae) extended its host range, usually restricted to crucifers, to sugar snap- and snowpeas (Pisum sativum L.) (Löhr, 2001). Due to the degree of specialization and behavioural differences in host location of its major indigenenous parasitoids this host shift was expected to have different effects on members of the associated parasitoid guild. Laboratory studies on parasitation and searching behaviour of one of the most important local parasitoids, Diadegma mollipla (Holmgren) (Hymenoptera: Ichneumonidae), and studies on the newly introduced exotic parasitoid Diadegma semiclausum Hellen, showed widely different results on the original and the novel host plant (Chapter 2 and 3). While the generalist D. mollipla parasitized more DBM larvae on snowpeas as compared to cabbage, parasitation levels of the specialist $D$. semiclausum were significantly higher on cabbage. Differential use of host location cues of these parasitoids were assumed to be the main reason for the differences in parasitation (Chapter 3). But how would the situation be in the field? The number of parasitoids attacking P. xylostella in Kenya is small and most species are 
considered relative generalists. We predicted, that generalist parasitoid species like $D$. mollipla would be able to find their host on snowpeas whereas crucifer-specific parasitoids would not. Over a period of 9 months between February 2003 and November 2003 weekly collections of DBM in cabbage and snowpeas were conducted in order to identify local parasitoid species and their abundance in both crops. Diadegma semiclausum was introduced to Kenya as part of a biocontrol programme of DBM in crucifers. It was released in snowpeas in November 2003 and its impact on DBM was recorded until July 2004.

\subsection{Material and Methods}

\subsubsection{Experimental fields}

The experimental site was located in an area of $800 \mathrm{~m}^{2}$ on a horticultural farm in Naivasha, Nakuru District in the Rift Valley in Kenya [Altitude: 1900m, 0044'98S, $\left.036^{\circ} 26^{\prime} 27 \mathrm{E}\right]$. Eight plots of $10 \mathrm{~m} \times 5 \mathrm{~m}$ size were established and planted simultaneously with kale (Brassica oleracea acephala L. var. Thousandheaded) on one half of the plots and snowpea (Pisum sativum var. Oregon sugar pod) on the other. Towards north and south the study area was bordered by different horticultural crops. During the study period tomatoes, french beans and Tagetes flowers were grown successively on the neighboring fields. On the other two sides pepper trees (Schinus molle L.) marked the border of the farm. The study plots were only sprayed once with a herbicide (Gamoxone, 150ml/151 water) at the beginning of the study. In Naivasha the soil is heavily infested with nematodes. Therefore an organophosphate (Nemacure) was incorporated at a rate of $5 \mathrm{~g} /$ plant into the holes for kale seedlings and snowpea seeds, respectively. Kale was transplanted after 3 weeks in the nursery (160 plants/plot). Snowpea was sown directly (10 rows/plot). DAP starter fertilizer was used ( $2 \mathrm{~g} / \mathrm{plant})$ for both crops. In the second half of the study it was replaced by organic manure. Further use of pesticides was avoided. An infection of snowpeas with the fungus 
Ascochyta end of May 2003 was treated once with an organic fungicide (Equation Pro $10 \mathrm{~g} / 151)$, but could not be controlled. The disease appeared in every new crop, but spraying was abandoned because of possible harmful effects on the DBM population and its natural enemies. Especially during the rainy seasons many pea plants were lost due to the disease. The plots were watered once a week using overhead irrigation, whenever necessary.

The study took place between $25^{\text {th }}$ of September 2002 and $6^{\text {th }}$ of July 2004 . Within this period both crops were constantly in the field (six subsequent crops for kale and eight for snowpea). New transplants and seeds were brought into the field before the old crop was harvested in order to ensure a quick infestation of the new crop with DBM. Plots were rotated within the area and half of the field was always left uncultivated for one growing period (approx. 3 months). New crops were transplanted or sown alternately on the plots, kales on former pea plots and vice versa.

The study farm was $20 \mathrm{~km}$ away from the farm where DBM was first observed on peas (see Löhr, 2001). During the first crop between 25.09.02 and 7.01.03 natural infestation of snowpeas with DBM was extremely low. Only 5 larvae were found in the entire period. In order to investigate the impact on natural enemies, a higher infestation level was required and eggs of the pea strain of DBM were brought from a laboratory culture into the field. On average, peas were infested with 500 eggs/plot every week, starting in Feb. 2003. Eggs had been laid on aluminium foils in the laboratory culture and strips of foil containing 50 to 100 eggs were randomly wrapped around pea plants. The artificial infestation was continued until the end of the study.

\subsubsection{Sampling}

20 randomly selected plants per plot were sampled weekly or biweekly. Larvae and pupae of DBM were recorded, collected and reared in the laboratory until adult stage. We did not look for egg parasitoids. Total number of emerging parasitoids was determined and species were identified. Unknown species were labeled and sent for identification to Georg Goergen at IITA (International Institute of Tropical Agriculture) 
in Benin. Other lepidopteran larvae were also collected and reared in the laboratory in order to check whether some emerging parasitoid species were identical with DBM parasitoids.

\subsubsection{Introduction of Diadegma semiclausum}

After a sampling period of 9 months (Nov. 2003) five pairs of D. semiclausum were released in each snowpea plot. Sampling continued as described above. The species was introduced from AVRDC (Asian Vegetable Research and Development Center) in Taiwan in 2002 and subsequently maintained in the laboratory at ICIPE in Nairobi. 


\subsection{Results}

Seven parasitoids species were collected from DBM on snowpeas (Table 6.1). Four species are parasitoids commonly found on DBM in crucifers in Kenya. The two braconids Cotesia sp. Cameron 1891 and Distatrix sp. Mason, 1981 (Microgasterinae), collected from DBM on snowpeas were not recovered from DBM on kales. Oomyzus sokolowskii Kurdjumov (Hym., Eulophidae) was the species most frequently found on the new host plant. The Apanteles sp. Foerster, 1862 (Hym., Braconidae: Microgasterinae) comprised at least two different species, but could not be further identified. Mean parasitism rate on snowpeas before the release of $D$. semiclausum was $2.3 \%$. After the release it rose to $4.0 \%$, due to a slight increase of numbers of $O$. sokolowskii and Cotesia sp. (Table 6.2). The exotic parasitoid only occurred sporadically on snowpeas. Diamondback moth on kales yielded six parasitoid species. Itoplectis sp. Foerster, 1869 (Hym., Ichneumonidae) was the only parasitoid that only occurred on kales. After its release, D. semiclausum established quickly on DBM on kales (Table 6.2). Numbers of DBM decreased from a total of 7571 collected over 9 months before the release to 1136 collected over 7 months after the release and the parasitism rate increased from an average of 25.6 to $75.7 \%$. Records of $D$. semiclausum on DBM during the pre-release period resulted from a release on a horticultural farm approx. $3 \mathrm{~km}$ away from the study plots in June 2003 (Table 6.1). As part of the biocontrol programme of DBM in Kenya mass releasing of this species took place along the Rift Valley and interfered marginally with our study on local parasitoids. However, it did not establish in large numbers. 
Table 6.1: Parasitoid guild of the diamondback moth, on kale and snowpea Feb. 2003Nov. 2003 in Naivasha, Rift Valley, Kenya (before release of D. semiclausum). For gregarious Oomyzus sokolowskii the number of parasitized DBM larvae is given and total number of emerged parasitoids is shown in brackets. Diadegma coccoons where the parasitoid failed to emerge are listed as Diadegma sp..

\begin{tabular}{|c|c|c|c|c|c|}
\hline kale & $\begin{array}{c}\text { Total } \\
\text { number }\end{array}$ & $\begin{array}{l}\text { \% total } \\
\text { para- } \\
\text { sitism }\end{array}$ & snowpea & $\begin{array}{l}\text { Total } \\
\text { numbe } \\
\mathbf{r}\end{array}$ & $\begin{array}{l}\text { \% total } \\
\text { para- } \\
\text { sitism }\end{array}$ \\
\hline Oomyzus sokolowskii & $\begin{array}{c}1332 \\
(9128)\end{array}$ & 68.6 & Oomyzus sokolowskii & $\begin{array}{c}17 \\
(147)\end{array}$ & 51.5 \\
\hline Diadegma mollipla & 400 & 20.6 & Apanteles sp. & 11 & 24.2 \\
\hline Apanteles sp. & 77 & 4.0 & Cotesia sp. & 3 & 9.1 \\
\hline Diadegma sp. & 60 & 3.1 & Brachymeria sp. & 2 & 6.1 \\
\hline Diadegma semiclausum & 52 & 2.7 & Diadegma mollipla & 1 & 3.0 \\
\hline Itoplectis $\mathrm{sp}$. & 19 & 1.0 & Diadegma semiclausum & 1 & 3.0 \\
\hline Brachymeria sp. & 2 & 0.1 & Distatrix sp. & 1 & 3.0 \\
\hline No. parasitized DBM & 1942 & & & 33 & \\
\hline Total no. DBM & 7571 & & & 1425 & \\
\hline Total \% parasitism & 25.6 & & & 2.3 & \\
\hline
\end{tabular}


Table 6.2: Parasitoid guild of Plutella xylostella in kale and snowpea Nov. 2003 - July 2004 in Naivasha, Rift Valley, Kenya (after release of D. semiclausum). For gregarious Oomyzus sokolowskii the number of parasitized DBM larvae is given and total number of emerged parasitoids is shown in brackets. Not emerged parasitoids from Diadegma coccoons were listed under Diadegma sp..

\begin{tabular}{lccllc}
\hline kale & $\begin{array}{c}\text { Total } \\
\text { numbe } \\
\text { r }\end{array}$ & $\begin{array}{c}\text { \% total } \\
\text { para- } \\
\text { sitism }\end{array}$ & snowpea & $\begin{array}{c}\text { Total } \\
\text { number }\end{array}$ & $\begin{array}{c}\text { \% total } \\
\text { para- } \\
\text { sitism }\end{array}$ \\
\hline Oomyzus sokolowskii & $\begin{array}{c}472 \\
(3117)\end{array}$ & 54.9 & Oomyzus sokolowskii & $29(179)$ & 46.8 \\
Diadegma semiclausum & 197 & 22.9 & Cotesia sp. & 13 & 21.0 \\
Diadegma sp. & 135 & 15.7 & Apanteles sp. & 10 & 16.1 \\
Diadegma mollipla & 31 & 3.6 & Diadegma semiclausum & 6 & 9.7 \\
Itoplectis sp. & 27 & 3.1 & Diadegma mollipla & 4 & 6.5 \\
Apanteles sp. & 6 & 0.7 & & & \\
Brachymeria sp. & 1 & 0.1 & & 62 & \\
\hline No. parasitized DBM & 860 & & & 1560 & \\
Total no. DBM & 1136 & & & 4.0 & \\
Total \% parasitism & 75.7 & & & & \\
\hline
\end{tabular}

Seasonal abundance of DBM and the number of parasitized larvae on snowpea are presented in Fig. 6.1. Peak numbers of DBM on snowpeas occurred before the release of D. semiclausum at the beginning of June until mid of July 2003 (21 to 58 larvae/20 plants)(Fig. 6.1A) and after release from early January 2004 until Februray 2004 (14 to 50 larvae/20 plants) (Fig. 6.1B). The number of parasitized DBM was very low throughout the study period (0 to 3.5 larvae), irrespective of the number of DBM in the field. With a constant infestation of snowpeas with DBM eggs the DBM population on snowpeas was kept artificially higher than it would have been under natural conditions. This was necessary in order to obtain a sizable population for parasitoid attack. Main 
causes of the fluctuations of the DBM population were heavy rains on the one hand, washing away foils with eggs or newly hatched larvae, and Ascochyta disease on the other. The disease started with brown spots on the lower leaves and spots and lesions spread rapidly to the stem and upper leaves. The infection caused the plant to dry up rapidly and prematurely. DBM larvae were not able to develop on dying plants. On kale, DBM population fluctuated mainly due to the age of the plants in the field. Very low numbers of 1 to 10 larvae/20 plants were only detected on freshly transplanted and young plants. The population peaked between end of June and end of August 2003, with up to 235 larvae/20 plants (Fig. 6.2A). The number of parasitized larvae fluctuated between 2 and 42/20 plants. After the release of D. semiclausum the DBM population on kale decreased continuously from 43 larvae/20 plants end of November 2003 to below 5 larvae/20 plants from mid Februray to the end of the study period in May 2004 (Fig. 6.2B). The number of parasitized larvae decreased simultaneously.
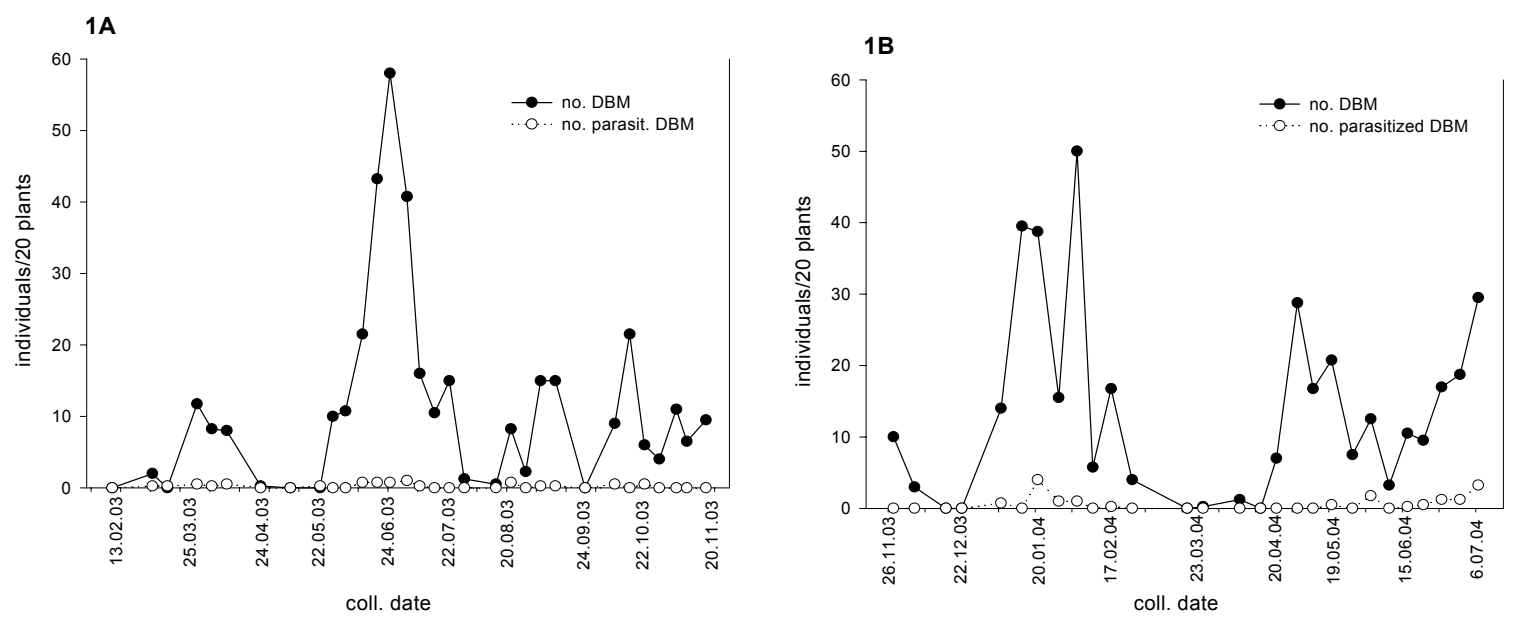

Fig. 6.1: Seasonal distribution of total and parasitized DBM larvae on snowpea A) before release of $D$. semiclausum, B) after release of $D$. semiclausum. 

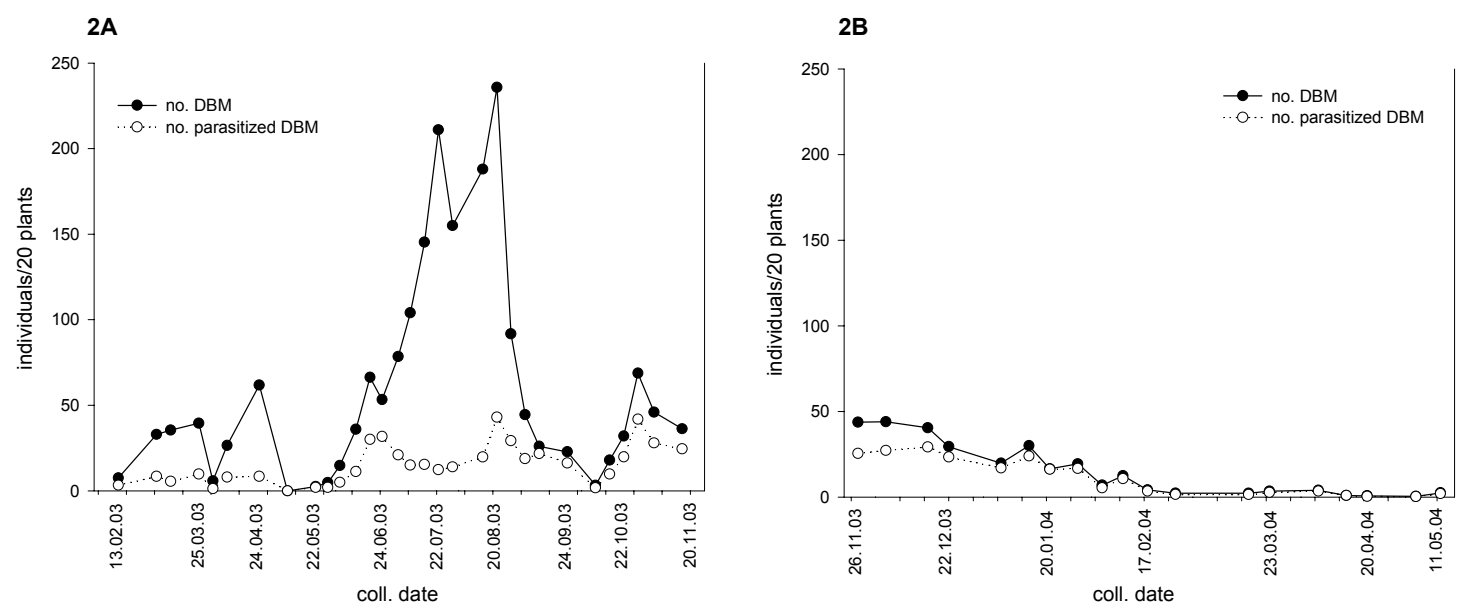

Fig. 6.2: Seasonal distribution of total and parasitized DBM larvae on kale A) before release of $D$. semiclausum, B) after release of $D$. semiclausum.

Occurrence of main parasitoids in snowpeas was sporadic (Fig. 6.3A). On average less than than 1 parasitized larvae/20 plants was collected and most frequently this larva was parasitized by $O$. sokolowskii. The braconids Apanteles sp. and Cotesia sp. were combined for the presentation in Fig. 6.3A. Their appearance was very scattered up to November 2003 and were found again in June 2004. In kale the main parasitoids occurred constantly throughout the duration of the study (Fig. 6.3B). Peaks of the most dominant $O$. sokolowskii and D. mollipla occurred simultaneously. Diadegma semiclausum remained in low numbers of less than 1 individual/20 plants after it had been released on a farm $3 \mathrm{~km}$ away from the study area. After its release in snowpeas plots it moved directly to the neighboring kale plots and parasitism of $O$. sokolowskii and D. mollipla decreased within 2 months to less than 1 individual/20 plants. Low numbers of $D$. semiclausum towards the end of the study period resulted from the decrease in DBM population after the release of the exotic parasitoid (Table 6.2). 

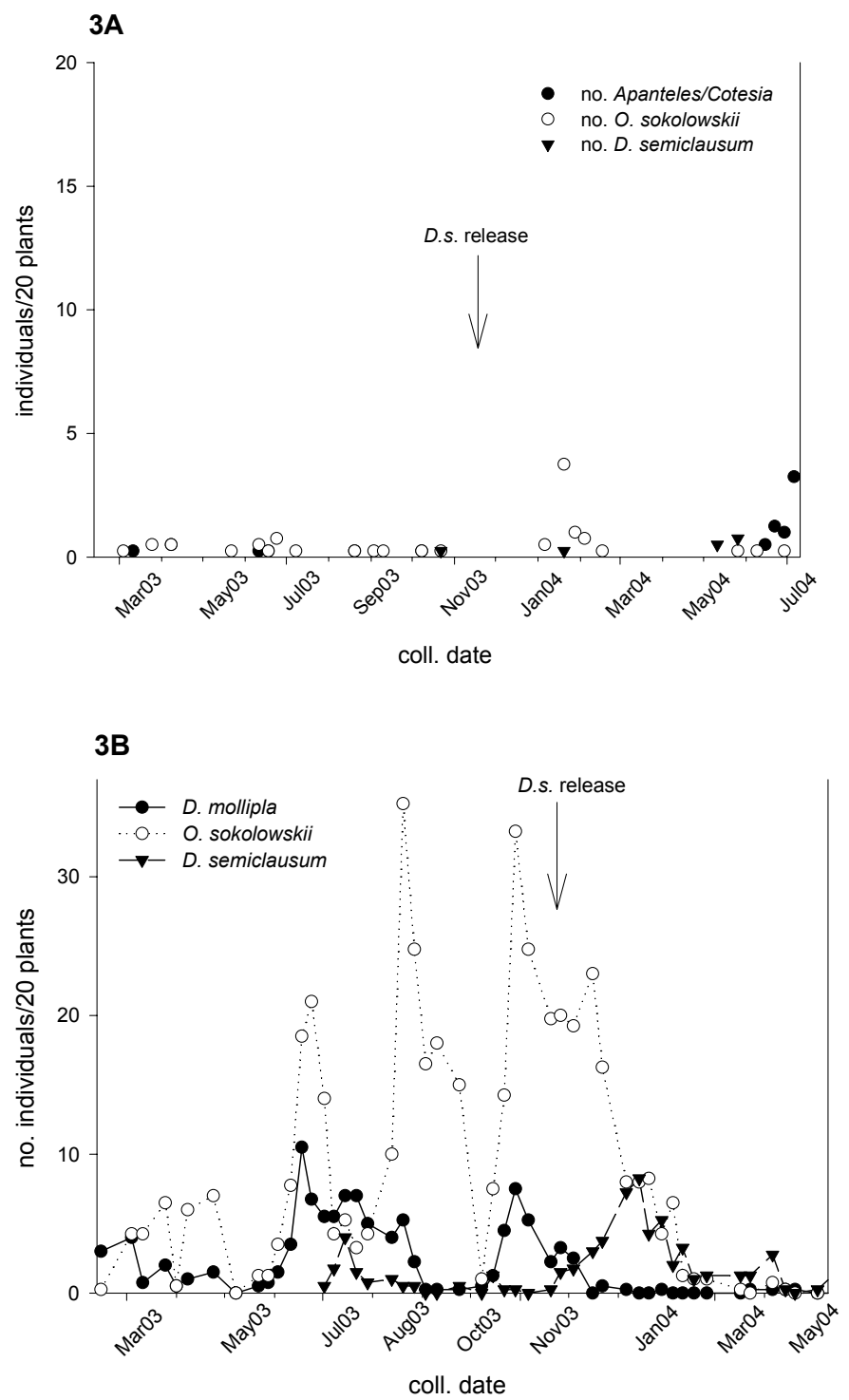

Fig 6.3: Seasonal distribution of main parasitoids.

For gregarious Oomyzus sokolowskii the number of parasitized DBM larvae is presented. A) on snowpea, B) on kale. 


\subsection{Discussion}

The inclusion of snowpea into its host plant range provided enemy-free space for the diamondback moth. On the newly acquired host plant, parasitoid occurrence was very sporadic and parasitism levels were extremely low. Parasitation in snowpeas seemed to be more a result of random rather than targeted movement of parasitoids. However, parasitoids were probably not the driving force for pioneering the new host plant but a mere consequence. DBM had shifted to non-cruciferous hosts before, especially in outbreak situations when populations on the original host plant were extremely high (Anonymous, 1971, Löhr and Gathu, 2002). Bigger and Fox (1997) showed that high density populations of DBM have broader host plant diets than low density populations, due to a wider distribution of eggs onto alternative plants. This might have facilitated the incorporation of peas into the diet of DBM. However, the introduction of the exotic D. semiclausum raised enemy pressure on DBM in crucifers substantially. The aquisition of novel host plants could be of future advantage for DBM in order to avoid parasitoid attack. On the other hand, the high impact of D. semiclausum will lead to low densities of DBM and could therfore prevent such host range expansions.

DBM parasitoids did not seem to recognize the novel host plant as a potential site for the occurrence of their host. Some DBM specific parasitoids have been shown to use crucifer-typical volatiles to locate their herbivore hosts (Shiojiri et al., 2000, Reddy et al., 2002, Ohara et al., 2003). The failure of D. semiclausum to establish on DBM on snowpeas was probably caused by its crucifer-related host searching behaviour (Ohara et al., 2003, Chapter 3). The most frequently found parasitoid in snowpeas, Oomyzus sokolowskii, is a cosmopolitan species which has been recorded as one of the major parasitoids of the diamondback moth worldwide (Waterhouse and Norris, 1987, Alam, 1992, Kfir, 1997, Liu et al., 2000). Although it has been introduced to various countries for biocontrol of DBM, only little is known about its biology (Ooi, 1988). To our knowledge host finding behaviour of $O$. sokolowskii has not been investigated yet and therefore the role of plant cues for host location is not known. Its relative abundance in snowpeas might result from a higher random movement and its overall dominance in the growing system. The indigenous D. mollipla was expected to occurr more 
frequently on DBM on snowpeas. Its host searching behaviour is not crucifer-related and parasitism on snowpeas was higher in laboratory experiments than on cabbage (see Chapter 3, Löhr and Rossbach, 2004). However, in the field it was hardly found on the novel host plant. Diadegma mollipla was gradually displaced from kales by $D$. semiclausum, but seemed to move to other hosts rather than to DBM on snowpeas. The species is considered a relative generalist and at least one other host is known, the potato tuber moth, Phthorimaea operculella (Zeller) (Lepidoptera: Gelechiidae) (Broodryk, 1971). The species-richness within the genus Apanteles sp. did not allow a differentiation between the specimen collected in the study site. We assume that there are at least two different species. The most frequent Apanteles sp. is more abundant in the hot and semi-arid areas of Kenya (Gichini pers. comm.). It is an undescribed species (Delvare, pers. comm.) and its biology is unknown. The Cotesia sp. that parasitized DBM only on snowpeas was first assumed to be Cotesia plutellae. The species was not recorded for Kenya. Its absence in kales was suspicious and our original assumption was not confirmed.

Other factors than host searching behaviour could have contributed to the low enemy impact on snowpeas. Lower population densities of DBM on snowpeas and the necessity to keep this population artificially in the field as well as the unhealthy state of plants might have led to low parasitation levels. After its outbreak in 1999 and 2000, serious damage levels of DBM on peas have not occurred up to date. But the adaptability of DBM to snowpeas was demonstrated by Löhr and Gathu (2002). Within four generations on snowpea survival of a cabbage strain of DBM was equally as good on both host plants. The relatively easy host plant adaption combined with an avoidance of enemy attack, especially by D. semiclausum, are factors that could facilitate host plant shifts of DBM in the future. 


\section{CHAPTER 7}

\section{General Discussion}

The use of a new host plant of the diamondback moth affected performance, development and searching behaviour of the two investigated parasitoids. Diadegma mollipla, which is regarded as a relative generalist, parasitized significantly more DBM larvae on snowpeas than on cabbage. The DBM-crucifer complex did not seem to be the preferred host-plant association. Host location studies in the Y-tube olfactometer showed, that D. mollipla females walked randomly when they had a choice between cabbage-related odours and clean air, whereas DBM-infested snowpea, as well as PTM-infested potatoes were preferred over clean air, indicating that D. mollipla has a broader host range on a variety of plants. It is assumed that generalist natural enemies use general chemical cues that are present in all hosts or their respective food plants (Vet and Dicke, 1992, Godfray, 1994). Accordingly, D. mollipla is either attracted to a non-specific blend of volatiles from a variety of hostinfested plants, or it uses other shared cues present in several hosts or host-plant complexes. Since D. mollipla is less dependent on plant volatiles for its host location, DBM feeding on a different plant does not necessarily mean a loss of cues, although the nature of cues that mediate in its host location process remain unknown. On the other hand, D. semiclausum relies on volatiles associated with cabbage, DBM's original host, but not with snowpeas. The relative indifference towards DBM-infested snowpea and the avoidance of the larvae of the pea strain implies lack of attractive volatiles and their precursors from this plant. Ohara et al. (2003) had already demonstrated that the specialisation of D. semiclausum on DBM is facilitated by crucifer-typical volatiles (perhaps derivates of glucosinolate), which are used by the parasitoid as cues for locating its host. Therefore a host shift to a different plant family leads to a loss of host location cues for D. semiclausum, and this seriously affects its searching efficiency.

Consequently, on a new host plant, efficiency of $D$. semiclausum as a biocontrol agent is greatly reduced. Parasitation levels on snowpeas were significantly lower than on cabbage. We therefore attempted to condition this parasitoid to locate and parasitize its host on the new plant. The recognition of host finding cues underlies a 
certain plasticity in order to cope with a changing environment and ensure survival (Turlings et al, 1993, Monge and Cortesero, 1996). And olfactory learning is one way to enable parasitoids to modify their behavioural responsiveness to host and plant- derived odours (Vet and Groenewold, 1990, Turlings et al, 1993). Parasitation and the level of attraction to snowpeas could be increased within three generations of rearing D. semiclausum on larvae on this host plant. However, the change in responsive behaviour came with some costs. Pupal mortality of pea-reared parasitoids increased, and an extreme shift in sex ratio to males reduced reproductive success. The biased sex ratio could be attributed to the plant quality, e.g. nutrient content, secondary compounds as demonstrated for other species by Godfray, 1994, Fox et al., 1996 and Campan and Benrey, 2004.

Only few studies about the effect of host plants on interspecific competition between parasitoids exist (Iwao et al., 2001). Due to differential host location abilities competitiveness of the two parasitoids was assumed to change on the new host plant. On cabbage, D. semiclausum clearly out-competed D. mollipla, producing $73 \%$ of total progeny (Sithole, 2004). Results from the laboratory implied an advantage for D. mollipla over D. semiclausum on snowpea as compared to cabbage. We assumed that this species would find its niche on snowpea whereas D. semiclausum remained predominant on cabbage. But under more natural conditions in the greenhouse cages D. mollipla performed much poorer than confined to small containers. Irrespective of the number of parasitoids or available larvae, it produced significantly less progeny on snowpeas than its competitor. However, D. mollipla still performed slighly better on snowpea than on cabbage and might be able to parasitize on snowpeas even in the presence of the overall predominant $D$. semiclausum, especially when it occurs in higher numbers. Maybe the overall dominance of $D$. semiclausum could be explained with chemical interference, although we have no evidence for it. Chemical interference and host discrimination due to external markers, left on the surface of newly parasitized larvae (Gauthier et al., 1999), pheromones (Mackauer, 1990) or a characteristic smell of adults (Infante, 2001) could be demonstrated for other parasitoid species.

As a conclusion from the studies on the two Diadegma species, their differential degree of specialization and behavioural differences in host location, the host plant 
shift of DBM was expected to have different effects on members of its entire parasitoid guild. The number of parasitoids attacking DBM in Kenya is small and most species are considered relative generalists. However, most local parasitoids did not seem to recognize the novel host plant as a potential site for the occurrence of their host. They attacked DBM on snowpeas only sporadically and in very low numbers. The most frequent species was Oomyzus sokolowskii Kurdjumov, a cosmopolitan DBM parasitoid (Kfir, 1997, Liu et al., 2000). The inclusion of snowpea into its host plant range provided enemy-free space for the diamondback moth. Reduction of natural enemy attack through aquisition of a new host plant has been demonstrated by various authors (Feder et al., 1995, Gratton and Welter, 1999, Gross et al., 2004, Mulatu et al., 2004). Diadegma mollipla was expected to occur more frequently in snowpeas, but it was hardly found on the novel host plant. It was gradually displaced from kales by $D$. semiclausum, but seemed to move to other hosts rather than to DBM on snowpeas. The field studies were conducted within a very short time after DBM was first found on snowpeas. After the outbreak in 1999 DBM has not occurred in such high numbers on snowpeas anymore. That might have given parasitoids little chance to adapt to a new host plant. 


\section{References}

Abbas, M.S.T, 1988. Biological and ecological studies on Diadegma semiclausum Hellen (Hym., Ichneumonidae), a larval parasite of the diamondback moth, Plutella xylostella (L.) (Lep., Plutellidae) in Egypt. Anz. Schaedlingskde, Pflanzenschutz, Umweltschutz 61: 1-2.

Agboka, K., Schulthess, F., Chabi-Olaye, A., Labo, I., Gounou, S. and Smith, H. 2002. Self-, intra-, and interspecific host discrimination in Telenomus busseolae Gahan and T. isis Polaszek (Hymenoptera: Scelionidae), sympatric egg parasitoids of the African cereal stemborer Sesamia clasmistis Hampson (Lepidoptera: Noctuidae). J. Ins. Behav. 15: 1-12.

Agelopoulos, N.G. and M.A. Keller, 1994. Plant-natural enemy association in the tritrophic system, Cotesia rubecula-Pieris rapae-Brassicaceae (Cruciferae): I. Sources of infochemicals. J. Chem. Ecol. 20: 1725-1734.

Akol, A.M., 2001. Effects of two neem insecticide formulations of the trophic interactions between the diamondback moth, Plutella xylostella (L.)(Lepidoptera:Plutellidae) and its parasitoids. PhD Dissertation. Kenyatta University, Department of Agricultural Entomology Nairobi, Kenya, 127 pp.

Akol, A.M., P.G.N. Njagi, S. Sithanantham and J.M. Mueke 2003. Effects of two neem insecticide formulations on the attractiveness, acceptability and suitability of diamondback moth larvae to the parasitoid, Diadegma mollipla (Holmgren) (Hym., Ichneumonidae). J. Appl. Ent. 127: 325-331.

Alam, M.M. 1992. Diamondback moth and its natural enemies in Jamaica and some other Caribbean islands.- In: Talekar, N.S. (Ed.): Diamondback moth and other crucifer pests. Proc. 2nd Inter. workshop 10-14 Dec, Tainan, Taiwan, AVRDC: 233-243.

Anonymous 1971. Outbreaks and new records. FAO Plant Prot. Bull. 19: 89-92.

Auger, J., Lecomte, C. Paris, J. and Thibout, E. 1989. Identification of leek moth and DBM frass volatiles that stimulate the parasitoid Diadromus pulchellus. J. Chem. Ecol. 15: 1391-1398.

Azidah, A.A., M.G. Fitton and Quicke, D.L.J. 2000. Identification of the Diadegma species (Hymenoptera: Ichneumonidae, Campopleginae) attacking the diamondback moth, Plutella xylostella (Lepidoptera: Plutellidae). Bull. Entomol. Res. 90: 375-389.

Barbosa, P., Saunders, J.A., Kemper, J., Trumbule, R., Olechno, J. and Martinat, P. 1986. Plant allelochemicals and insect parasitoids. Effects of nicotine on Cotesia congregata (Say) (Hymenoptera: Braconidae) and Hyposoter annulipes (Cresson) (Hymenoptera: Ichneumonidae). J. Chem. Ecol. 12: 13191328.

Barron, A.B. 2001. The life and death of Hopkins' host-selection principle. J. Ins. Behav. 14: 725-737. 
Beck, N.J. and P.J Cameron,. 1990. Comparison of lepidopteran pest populations and their parasitoids in three vegetable brassicas. Proc. 43rd NZ Weed and Pest Control Conf., Palmerston North, NZ: 21-25.

Benrey,B., Denno, R.F., Kaiser, L. 1997. The influence of plant species on attraction and host acceptance in Cotesia glomerata (Hymenoptera: Braconidae). J. Ins. Behav. 10 (5): 619-630.

Bernays, E. and Graham, M. 1988. On the evolution of host specifity in phytophagous arthropods. Ecology 69 (4): 886-892.

Bernays, E.A. and R.F. Chapman, 1994. Host-plant selection by phytophagous insects. Chapman \& Hall, New York

Bigger, D.S and Fox, L.R. 1997. High density populations of diamondback moth have broader host-plant diets. Oecologia 112: 179-186.

Billquist, A. and Ekbom, B. 2001 Effects of host plant species on the interaction between the parasitic wasp Diospilus capito and pollen beetles (Meligethes spp.) Agricult. And Forest Entomol. 3: 147-152

Bjorksten, T.A. and Hoffmann, A.A. 1995. Effects of pre-adult and adult experience on host acceptance in choice and non-choice tests in two strains of Trichogramma. Ent. exp. appl. 76: 49-58.

Bogahawatte, C.N.L. and Van Emden, H.F. 1996. The influence of the host plant of diamondback moth (Plutella xylostella) on the plant preferences of its parasitoid Cotesia plutellae in Sri Lanka. Physiol. Entomol. 21: 93-96.

Bottrell, D.G., Barbosa, P. and Gould, F. 1998. Manipulating natural enemies by plant variety selection and modification: A realistic strategy?. Annu. Rev. Entomol. 43: 347-367.

Bradburne, R.P. and Mithen, R. 2000. Glucosinolate genetics and the attraction of the aphid Diaeretiella rapae to Brassica. Proc. R. Soc. Lond. B Biol. Sci. 267: 89-95.

Broodryk, S.W., 1971. The biology of Diadegma stellenboschense (Cameron) (Hymenoptera: Ichneumonidae), a parasitoid of potato tuber moth. J. ent. Soc. sth. Afr. 34: 413-423.

Brown, J.M., Abrahamson, W.G., Packer, R.A. and Way, P.A. 1995. The role of natural enemy escape in a gallmaker host plant shift. Oecologia 104: 52-60.

Bush, G.L. 1994. Sympatric speciation in animals: new wine in old bottles. Trends in Ecology and Evolution 9 (8): 285-288.

Campan, E. and Benrey, B. 2004. Behavior and performance of a specialist and a generalist parasitoid of bruchids on wild and cultivated beans. Biol. Contr. 30: 220-228.

Campos, F., Domskov, N., Arnason, J.T., Philogène, B., Atkinson, J., Morand, P. and Werstiuk, N.H. 1990. Biological effects and toxicokinetics of DIMBOA in Diadegma terebrans (Hymenoptera: Ichneumonidae), an endoparasitoid of Ostrinia nubilalis (Lepidoptera: Pyralidae). J. Econom. Ent. 83: 356-360.

Charnov, E.L., Los-den Hartogh, R.L., Jones, W.T. and van den Assen, J. 1981. Sex ratio evolution in a variable environment. Nature 289: 27-33. 
Chua, T.H., Gonzales, D. and Bellows, T. 1990. Searching efficiency and multiparasitism in Aphidius smithi and Aphidius ervi (Hymenoptera, Aphidiidae), parasites of pea aphid, Acyrthosiphon pisum (Homoptera, Aphididae). J. Appl. Ent. 110: 101-106.

Cortesero, A.M., Stapel, J.O. and Lewis, W.J. 2000 Understanding and manipulating plant attributes to enhance biological control. Biol. Contol 17: 35-49

Cronin, J.T. and Abrahamson, W.G. 2001. Do parasitoids diversify in response to host-plant shifts by herbivorous insects? Ecol. Entomol. 26: 347-355.

Davis, A.J., 1987. Effects of experiences and kairomones on searching behaviour of Diadegma eucerophaga (Ichneumonidae) for Plutella xylostella (Plutellidae) on Brassica plants. J. Med. Fac. Landbouww. Rijksuniv. Gent 52 (2a): 403411.

Dicke, M. 1994. Local and systemic production of volatile herbivore induced terpenoids: their role in plant-carnivore mutualism. J. Plant Physiol. 143: 465472.

Dicke, M. and van Loon, J.J.A. 2000. Multitrophic effects of herbivore-induced plant volatiles in an evolutionary context. Entomol. exp. appl. 97: 237-249.

Drost, Y.C., W.J. Lewis, P.O. Zanen, and M.A. Keller, 1986. Beneficial arthropod behavior mediated by airborne semiochemicals I.. J. Chem. Ecol. 12: 12471262.

Ehrlich, P.R. and P.H. Raven, 1964. Butterflies and plants: a study in coevolution. Evolution 18: 586-608.

Feder, J.L. 1995. The effects of parasitoids on sympatric host races of Rhagoletis pomonella (Diptera: Tephritidae). Ecology 76 (3): 801-813.

Fox, L.R., D.K. Letourneau, J. Eisenbach and S. Nouhuys Van, 1990. Parasitism rates and sex ratios of a parasitoid wasp: effects of herbivore and plant quality. Oecologia 83: 414-419.

Fox, L.R., K.M. Kester and J. Eisenbach, 1996. Direct and indirect responses of parasitoids to plants: sex ratio, plant quality and herbivore diet breadth. Entomol. Exp. Appl. 80: 289-292.

Gandolfi, M., Mattiacci, L. and Dorn, S. 2003. Preimaginal learning determines adult response to chemical stimuli in a parasitic wasp. Proc. R. Soc. Lond. B, Biol. Sci. 270: 2623-2629.

Gauthier, N., Sanon, A., Monge, J.P. and Huignard, J. 1999. Interspecific relations between two sympatric species of Hymenoptera, Dinarmus basalis (Rond) and Eupelmus vuilleti (Crw), ectoparasitoids of the bruchid Callosobruchus maculatus (F). J. Ins. Behav. 12: 399-413.

Geervliet, J.B.F., Vet, L.E.M. and Dicke, M. 1994. Volatiles from damaged plants as major cues in long-range host searching by the specialist parasitoid Cotesia rubecula. Entomol exp. appl. 73: 289-297.

Geervliet, J.B.F., Vet, L.E.M. and Dicke, M. 1996. Innate responses of the parasitoids Cotesia glomerata and C. rubecula (Hymenoptera: Braconidae) to volatiles from different plant-herbivore complexes. J. Ins. Behav. 9: 525-538. 
Godfray, H.C.J. 1994. Parasitoids. Behavioral and evolutionary ecology. Monographs in Behavior and Ecology. Princton Univ. Press: 1-473.

Gratton, C. and Welter, S.C. 1999. Does "enemy-free space" exist? Experimental host shifts of an herbivorous fly. Ecology 80 (3): 773-785.

Gross, J., Fatouros, N.E.; Neuvonen, S. and Hilker, M. 2004. The importance of specialist natural enemies for Chrysomela lapponica in pioneering a new host plant. Ecol. Entomol. 29: 584-593.

Gupta, V.K. 1974. Studies on certain porizontine ichneumonids reared from economic hosts (parasitic hymenoptera). Oriental Insects 3: 99-116.

Kfir, R. 1998. Origin of diamondback moth (Lepidoptera: Plutellidae). Annals of the Entomological Society of America 91: 164-167.

Harcourt, D. G. 1956. Biology of the diamondback moth, Plutella maculipennis (Curt.) (Lepidoptera:Plutellidae), in Eastern Ontario. I. Distribution, economic history, synonomy, and general description. 37th Rep. Quebec Soc. Prot. Plants, 1955, pp. 155-160.

Harvey, J.A., M.A. Jervis, G.J.Z. Gols, N. Jiang, and L.E.M. Vet, 1999. Development of the parasitoid, Cotesia rubecula (Hymenoptera: Braconidae) in Pieris rapae and P. brassicae (Lepidoptera: Pyralidae): evidence for host regulation. J. Insect Physiol. 45 173-182.

Havill, N.P. and Raffa, K.F. 2000. Compound effects of induced plant responses on insect herbivores and parasitoids: implications for tritrophic interactions. Ecol. Entomol. 25: 171-179.

Hérard, F., Keller, M.A., Lewis, W.J. and Tumlinson, J.H. 1988. Beneficial arthropod behavior mediated by airborne semiochemicals IV.- Influence of host diet on host-oriented flight chamber responses of Microplitis demolitor Wilkinson. J. Chem. Ecol. 14 (7): 1597-1606.

Idris, A.B. and E. Grafius, 1996. Effects of wild and cultivated host plants on oviposition, suvival, and development of diamondback moth (Lepidoptera: Plutellidae) and its parasitoid Diadegma insulare (Hymenoptera: Ichneumonidae). Environ. Entomol. 25: 825-833.

Infante, F., Mumford, P., Barrera, J. and Fowler, S. 2001 Interspecific competition between Cephalonomia stephanoderis and Prorops nasuta (Hym., Bethylidae), parasitoids of the coffee berry borer, Hypothenemus hampei (Col., Scolytidae).- J. Appl. Ent. 125: 63-70.

Iwao, K., Nakamura, S. and Ohsaki, N. 2001. Plant-based host preference and larval competition of the tachinid parasitoid Epicampocera succincta. Popul. Ecol. 43: 149-155.

Jeffries, M.J. and Lawton, J.H. 1984. Enemy free space and the structure of ecological communities. Biol. J. Linnean Soc. 23: 269-286.

Kfir, R. 1997. Parasitoids of Plutella xylostella (Lep.: Plutellidae) in South Africa: an annotated list. Entomophaga 42 (4): 517-523.

Kroschel, J. 1993. Integrierter Pflanzenschutz im Kartoffelanbau der Republik Yemen unter besonderer Berücksichtigung der integrierten biologischen 
Bekämpfung der Kartoffelmotte (Phthorimaea operculella (Zeller)). PLITS 11 (1), Josef Margraf, Weikersheim. p. 241.

Liu, S. and Jiang, L. 2003. Differential parasitism of Plutella xylostella (Lepidoptera: Plutellidae) larvae by the parasitoid Cotesia plutellae (Hymenoptera: Braconidae) on two host plants. Bull. Entomol. Res. 93: 65-72.

Liu, S-S., Wang, X.G., Guo, S-J., He, J-H. and Shi, Z-H. 2000. Seasonal abundance of the parasitoid complex associated with the diamondback moth, Plutella xylostella (Lepidoptera: Plutellidae) in Hangzhou, China. Bull. Entomol. Res. 90: 221-231.

Lloyd, D.C. 1940. Host selection by hymenopterous parasites of the moth Plutella maculipennis Curtis. Proceedings of the Royal Society of London Series B 128: $451-484$.

Löhr, B. and Gathu, R. 2002. Evidence of adaptation of diamondback moth, Plutella xylostella (L.), to pea, Pisum sativum L.. Insect Sci. Applic. 22 (3): 161-173.

Löhr, B. and Rossbach, A. 2004. Diamondback moth, Plutella xylostella (L.) on peas in Kenya: impact of the host shift on the pest and its parasitoid. 4th Int. Workshop on the Management of DBM and other crucifer pests, Melbourne 2001, pp 133-139.

Löhr, B., 2001. Diamondback moth on peas, really. Biocontrol News and Information 19: 38N-39N.

Mackauer, M, Sequeira, R. and Otto, M. 1997. Growth and development in parasitoid wasps: Adaptation to variable host resources. Ecol. Studies 130: 191-203.

Mackauer, M. 1990. Host discrimination and larval competition in solitary endoparasitoids. In: M. Mackauer, L.E. Ehler and J. Roland (eds), Critical issues in Biological Control. Intercept, Andover, Hants, pp. 41-62.

McBrien, H. and Mackauer, M. 1990. Heterospecific larval competition and host discrimination in two species of aphid parasitoids: Aphidius ervi and Aphidius smithi. Ent. exp. appl. 56: 145-153.

Monge, J.P. and Cortesero, A.M. 1996. Tritrophic interactions among larval parasitoids, bruchids, and Leguminosae seeds; influence of pre- and postemergence learning on parastoids' response to host and host-plant cues. Ent. exp. appl. 80: 293-296.

Mulatu, B., Applebaum, S.W. and Coll, M. 2004. A recently acquired host plant provides an oligophagous insect herbivore with enemy-free space. Oikos 107: 231-238.

Murchie, A.K., L.E. Smart and I.H. Williams, 1997. Responses of Dasineura brassicae and its parasitoids Platygaster subuliformis and Omphale clypealis to field traps baited with organic isothiocyanates. J. Chem. Ecol. 23: 917-926.

Ngi-Song, A.J., Njagi, P.G.N., Torto, B. and Overholt, W.A. 2000. Identification of behaviourally active components from maize volatiles for the stemborer parasitoid Cotesia flavipes Cameron (Hymenoptera: Braconidae). Insect Sci. Appl.: 20 181-189. 
Ngi-Song, A.J., Overholt, W.A., Njagi, P.G.N., Dicke, M., Ayertey, J.N. and Lwande, W. 1996. Volatile infochemicals used in host and host habitat location by Cotesia flavipes Cameron and Cotesia sesamiae (Cameron) (Hym.; Braconidae), larval parasitoids of stemborers on Graminae. J. Chem. Ecol. 22: 307-323.

Odour, G.I., Loehr, B. and Seif, A.A., 1996. Seasonality of the major cabbage pests and incidence of their natural enemies in central Kenya. In: Sivapragasam, W.H., Kole, A.K. Hassan and G.S. Lim (eds), The management of diamondback moth and other crucifer pests. Proc. 3rd Int. workshop on DBM, Kuala Lumpur, Malaysia 29 Oct-1 Nov 1996. pp. 37-42.

Ohara, Y., Akio, T. and Takabayashi, J. 2003 Response to host-infested plants in females of Diadegma semiclausum Hellen (Hymenoptera: Ichneumonidae). Appl. Entomol. Zool. 38 (1): 157-162

Ooi, P.A.C. 1988. Laboratory studies of Tetrastichus sokolowskii. Entomophaga 33: 145-152.

Pijls, J.W.A.M., Hofker, K.D., Van Staalduinen, M.J. and Van Alphen, J.J.M. 1995. Interspecific host discrimination and competition in Apoanagyrus (Epidinocarsis) lopezi and $A$. (E.) diversicornis, parasitoids of the cassava mealybug Phenacoccus manihoti. Ecol. Entomol. 20: 326-332.

Pivnick, K.A., 1993. Response of Meteorus leviventris (Hymenoptera: Braconidae) to mustard oils in field trapping experiments. J. Chem. Ecol. 19: 2075-2079.

Pivnick, K.A., Blair, J.J. and Slater, G.P. 1994. Identification of olfactory cues used in host-plant finding by diamondback moth, Plutella xylostella (Lepidoptera: Plutellidae). J. Chem. Ecol. 20. 1407-1427.

Potting, R.P.J., Poppy, G.M. and Schuler, T.H. 1999. The role of volatiles from cruciferous plants and pre-flight experience in the foraging behaviour of the specialist parasitoid Cotesia plutellae. Entomol. exp. appl. 93: 87-95.

Price, P.W., C.E. Bouton, P. Gross, B.A. McPheron, J.N. Thompson and A.E. Weis, 1980. Interactions among three trophic levels: Influence of plants on interactions between insect herbivores and natural enemies. Annu. Rev. Ecol. Syst. 11: 41-65.

Reddy, G.V.P., Holopainen, J.K. and Guerrero, A. 2002. Olfactory responses of Plutella xylostella natural enemies to host pheromone, larval frass and green leaf cabbage volatiles. J. Chem. Ecol. 28: 131-143.

Reitz, S.R. and Trumble, J.T. 2002. Competitive displacement among insects and arachnids. Annu. Rev. Entomol. 47: 435-465.

Roth, S., Knorr, C. and Lindroth, R.L. 1997. Dietary phenolics affects performance of the gypsy moth (Lepidoptera: Lymantriidae) and its parasitoid Cotesia melanoscela (Hymenoptera: Braconidae). Environ. Ent. 26: 668-671.

Shiojiri, K., Takabayashi, J., Yano S. and Takafuji, A. 2000. Flight response of parasitoids toward plant-herbivore complexes: a comparative study of two parasitoid-herbivore systems on cabbage plants. Appl. Entomol. Zool. 35 (1): 87-92. 
Sithole, R. 2004. Life history parameters of Diadegma mollipla (Holmgren), competition with Diadegma semiclausum Hellen (Hymenoptera: Ichneumonidae) and spatial and temporal distribution of the host, Plutella xylostella (L.) and its indigenous parasitoids in Zimbabwe.

Steidle, J.L.M. and van Loon, J.J.A. 2003. Dietary specialization and infochemical use in carnivorous arthropods: testing a concept. Entomol. exp. appl. 108 (3) 133-148.

Steidle, J.L.M., Steppuhn, A. and Ruther, J. 2003. Specific foraging kairomones used by a generalist parasitoid. J. Chem. Ecol. 29 (1): 131-143.

Strand, M.R., J.A. Johnson and J.R. Culin, 1988. Developmental interactions between the parasitoid Microplitis demolitor (Hymenoptera: Braconidae) and its host Heliothis virescens (Lepidoptera: Noctuidae). Ann. Entomol. Soc. Amer. 81: 822-830.

Strong, D.R. 1979. Biogeographic dymanics of insect-host plant communities. Annu. Rev. Entomol. 24: 89-119.

Sznajder, B. and Harvey, J.A. 2003 Second and third trophic level effects of differences in plant species reflect dietary specialisation of herbivores and their endo parasitoids.- Ent. exp. appl. 109 73-82

Takabayashi, J., Sato, Y., Horikoshi, M., Yamaoka, R., Yano, S., Ohsaki, N. and Dicke, M. 1998. Plant effects on parasitoid foraging: differences between two tritrophic systems. Biol. Contol 11 (2): 97-103.

Talekar, N.S. and A.M. Shelton, 1993. Biology, ecology, and management of the diamondback moth. Annu. Rev. Entomol. 38: 275-301.

Talekar, N.S. and J.C. Yang, 1993. Influence of crucifer cropping system on the parasitism of Plutella xylostella (Lep., Yponomeutidae) by Cotesia plutellae (Hym., Braconidae) and Diadegma semiclausum (Hym., Ichneumonidae). Entomophaga 38: 541-550.

Talekar, N.S., Yang, H.C., Lee, S.T., Chen, B.S. and Sun, L.Y. 1985. Annotated bibliography of diamondback moth. AVRDC Publ. 85-229. Shanhua, Taiwan, $469 \mathrm{pp}$.

Turlings, T.C.J., J.H. Tumlinson, F.J. Eller and W.J. Lewis, 1991. Larval-damaged plants: source of volatile synomones that guide the parasitoid Cotesia marginiventris to the microhabitat of its hosts. Entomol. Exp. Appl. 58: 75-82.

Turlings, T.C.J., Wäckers, F.L., Vet, L.E.M., Lewis, W.J. and Tumlinson, J.H. 1993. Learning of host-finding cues by hymenopterous parasitoids. In D.R. Papaj \& A.C. Lewis (eds), Insect learning. Chapman \& Hall, NY, p. 51-78.

Ullyett, G.C. 1943. Some aspects of parasitism in field populations of Plutella maculipennis Curt. J. Entomol. Soc. South Afr. 6: 65-80.

Venkatraman, T.V. 1964. Experimental studies on superparasitism and multiparasitism in Horogenes cerophaga (Grav.) and Hymenobosmina rapi (Cam.), the larval parasites of Plutella maculipennis (Curt.). Indian Journal of Entomology 26: 1-32. 
Verkerk, R.H.J. and Wright, D.J. 1996. Multitrophic interactions and management of the diamondback moth: a review. Bull. Entomol. Res. 86: 205-216.

Vet, L.E.M. and Dicke, M. 1992. Ecology of infochemical use by natural enemies in a tritrophic context. Annu. Rev. Entomol. 37: 141-172.

Vet, L.E.M. and Groenewold, A.W. 1990. Semiochemicals and learning in parasitoids. J. Chem. Ecol. 16 (11): 3119-3135.

Vinson, B.S. 1976. Host selection by insect parasitoids. Ann. Rev. Entomol. 21: 109133.

Wang, X.-G. and Keller, M.A. 2002. A comparison of the host-searching efficiency of two larval parasitoids of Plutella xylostella. Ecol. Entomol. 27: 105-114.

Wang, X.-G. and Messing, R.H. 2003. Intra-and interspecific competition by Fopius arisanus and Diachasmimorpha tryoni (Hymenoptera: Braconidae), parasitoids of tephritid fruit flies. Biol. Contol 27: 251-259.

Waterhouse, D.F. and Norris, K.R. 1987. Biological Control - Pacific Prospects. Inkata Press, Mebourne

Xu, J, Shelton, A.M. and Cheng, X. 2001. Comparison of Diadegma insulare (Hymenoptera: Ichneumonidae) and Microplitis plutellae (Hymenoptera: Braconidae) as biological control agents of Plutella xylostella (Lepidoptera: Plutellidae): Field parasitism, insecticide susceptibility, and host searching. J. Econ. Ent. 94: 14-20.

Yang, J.C., Chu, Y.I. and Talekar, N.S. 1994. Studies on the characteristics of parasitism of Plutella xylostella (Lep.: Plutellidae) by larval parasite Diadegma semiclausum (Hym.: Ichneumonidae). Entomophaga 39: 397-406.

Zaki, F.N., G. El-Saadany, A. Gomma and M. Saleh, 1998. Increasing rates of parasitism of the larval parasitoid Bracon brevicornis (Hym., Braconidae) by using kairomones, pheromones and a supplementary food. J. Appl. Ent. 122: 565-567. 


\section{Zusammenfassung}

Die Kohlmotte Plutella xylostella L. gilt als kosmopolitisch verbreiteter, spezifischer Schädling der Familie Brassicaceae und ist weltweit einer der grössten Schädlinge an Gemüsesorten aus dieser Familie. Im Rift Valley in Kenia wurde vor einigen Jahren eine Population der Kohlmotte auf Zuckererbsen entdeckt. Sie verursachte dort sehr grosse Schäden. Die Wirkung dieses Wirtspflanzenwechsels auf die assoziierten Parasitoide wurde untersucht. In Laborexperimenten wurden Parasitierungsraten, Entwicklungdauer und Überlebensraten sowie die Rolle der Wirtspflanze für die Wirtsfindung an zwei wichtigen Parasitoidenarten untersucht: die einheimische Diadegma mollipla (Holmgren), die als relativer Generalist angesehen wird, und die hochspezifische Diadegma semiclausum (Hellen), die für die biologische Kontrolle der Kohlmotte auf Brassica-Gemüse nach Kenia eingeführt wurde.

Diadegma mollipla war überraschenderweise effektiver auf der neuen Wirtspflanze als auf Kohl. Diadegma semiclausum parasitierte auf Kohl fressende Larven effizienter. Wirtsfindungsuntersuchungen mit Hilfe eines Y-Rohr Olfaktometer zeigten, dass die Spezialisierung von D. semiclausum durch Signale der cruciferen Wirtspflanze vermittelt wird, und nicht durch Signale von auf Erbsen fressenden Larven. Während flüchtige Substanzen von Brassicaceen keinen Hauptreiz für die Wirtsfindung von D. mollipla darstellt. Diese Unterschiede in Parasitierungs- und Wirtsfindungsverhalten ließen vermuten, dass sich das Konkurrenzverhalten der beiden Arten auf Erbsen im Vergleich zu Kohl ändert. Auf Kohl behauptet sich $D$. semiclausum eindeutig. Auf Erbsen, zumindest bei Experimenten in kleinen Behältern, parasitierte D. mollipla genauso gut oder sogar besser als D. semiclausum. Allerdings parasitierte D. semiclausum unter natürlicheren Bedingungen im Gewächshaus auf beiden Wirtspflanzen signifikant mehr Larven als ihr Konkurrent. Chemische Interferenz zwischen den beiden Arten ist vielleicht eine mögliche Erklärung dafür.

Olfaktorisches Erlernen von Stimuli gilt als Möglichkeit für die Fähigkeit von Parasitoiden ihre Reaktion auf Wirts- und Wirtspflanzenreize zu verändern. Im 
Hinblick auf eine Kontrolle der Kohlmotte auf Erbsen, wurde versucht, die Parasitierungsleistung von D. semiclausum auf Erbsen zu erhöhen. Eine Anpassung an eine neue Wirtspflanze durch das Erlernen von Schlüsselreizen könnte möglich sein. Die Schlupfwespe wurde für drei Generationen auf dem Erbsenstamm der Kohlmotte gezüchtet. Die Reaktion auf Reize von einer mit Larven infestierten Erbsenpflanze sowie die Parasitierungsrate konnte gesteigert werden. Allerdings zeigten die Generationen auf Erbsen eine reduzierte Fitness. Vor allem die extreme Verschiebung des Geschlechterverhältnisses auf die Seite der Männchen sorgte für eine starke Verschlechterung in der Reproduktion.

Um die Wirkung der ungewöhnlichen Wirtspflanze auf den gesamten Parasitoidenkomplex der Kohlmotte zu untersuchen, wurden in der Nähe der Farm, wo die Kohlmotte zuerst auf Erbsen gefunden wurde, Feldstudien durchgeführt. Erbsen schafften für die Kohlmotte einen Feind-freien Raum. Die einheimischen Schlupfwespenarten kamen nur sporadisch und in sehr geringer Zahl auf Erbsen vor. Die Freilassung von D. semiclausum führte $\mathrm{zu}$ einer Verringerung der Kohlmottenlarven auf Kohl, aber die Art konnte sich in den Erbsen nicht etablieren. Gleichzeitig reduzierte sich die Zahl der lokalen Parasitoidenarten. Mit der starken Konkurrenz durch D. semiclausum, wurde erwartet, dass D. mollipla ihre Niche auf dem Erbsenstamm der Kohlmotte finden würde. Allerdings erhöhte sich deren Zahl auf Erbsen nicht. Die Art wurde möglicherweise auf ihre anderen Wirte verdrängt.

Es konnte insgesamt gezeigt werden, dass die Wirtspflanze einen sehr starken Einfluss auf Parasitierung, Fitness und Wirtsfindungsverhalten hat, und somit ein Schädling mit der Aufnahme einer neue Wirtspflanze ins Frassrepertoire seinen Feinden zunächst erfolgreich ausweichen kann. Durch die Fähigkeit zur Anpassung von Parasitoiden an eine neue Wirtspflanze, können diese möglicherweise mit der Zeit ihrem Wirt nachfolgen. 


\section{Acknowledgements}

The study was conducted in the ICIPE-led project entitled "Biocontrol of diamondback moth in Eastern Africa", financed by the German Federal Ministry of Economic Cooperation and Development (BMZ).

Thanks to Bernhard Löhr for giving me the opportunity to conduct this research within the DBM-project. And thanks for a very pleasant working atmosphere.

I thank the German Research Foundation (DFG) for the financial support of myself and this research.

I also thank Hortitec Seeds Ltd. in Naivasha for providing me with an investigation plot and facilities. Special thanks to Livingston Makwata for the management of the study site.

And last but not least, I thank the entire DBM-Team for their support, their help and the great working atmosphere. Ahsante sana, Nicholas, Faith, Rafael, Gatama, Rose, Ruth, Caleb, Macharia. 


\section{Lebenslauf}

Name: Roßbach

Vorname: Andrea

Geburtsdatum: 3.1 .68

Geburtsort: Marl

Familienstand: ledig

Staatsangehörigkeit: deutsch

\section{Promotion}

03/02 - 01/06 Am Institut für Pflanzenpathologie und Pflanzenschutz an der Universität Göttingen und International Centre of Insect Physiology and Ecology in Nairobi, Kenia

Abschluss: Dr. agr. (summa cum laude)

Thema der Promotion: Einfluss des Wirtswechsels der Kohlmotte Plutella xylostella L. auf assoziierte Parasitoide in Kenia

\section{Hochschulstudium}

10/99-09/01 Agrarwissenschaften der Tropen und Subtropen an der Universität Göttingen. Schwerpunkte: Pflanzenschutz, Agrarentomologie

Abschluss: M.Sc. agr., Note: sehr gut $(1,1)$

10/89-6/96 Diplom-Biologie an der Universität Osnabrück Schwerpunkte: Ökologie, Entomologie

Abschluss: Dipl. Biol., Note: sehr gut $(1,5)$

\section{Berufsausbildung}

09/87 - 06/89 Berufsfachschule für Technische Assistenten in Olsberg, Abschluss: Biologisch-technische Assistentin, Note: gut $(1,8)$

\section{Schulbildung}

09/78 - 06/87 Gymnasium im Loekamp in Marl, Abschluss: Abitur

09/74-07/78 Grundschule an der Westfalenstrasse in Marl 